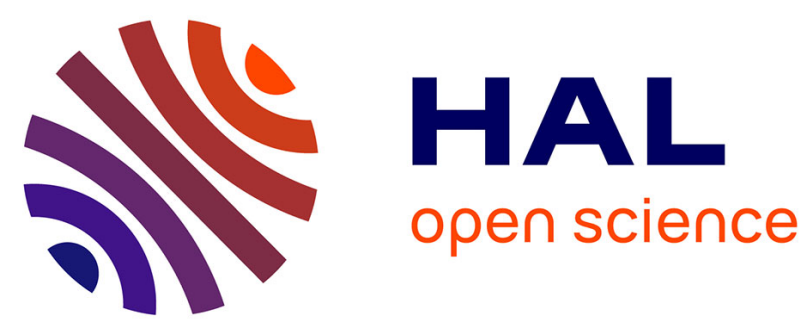

\title{
Typed binary trees and generalized dendrifom algebras
} Loïc Foissy

\section{To cite this version:}

Loïc Foissy. Typed binary trees and generalized dendrifom algebras. Journal of Algebra, 2021. hal02483607

\section{HAL Id: hal-02483607 https://hal.science/hal-02483607}

Submitted on 18 Feb 2020

HAL is a multi-disciplinary open access archive for the deposit and dissemination of scientific research documents, whether they are published or not. The documents may come from teaching and research institutions in France or abroad, or from public or private research centers.
L'archive ouverte pluridisciplinaire HAL, est destinée au dépôt et à la diffusion de documents scientifiques de niveau recherche, publiés ou non, émanant des établissements d'enseignement et de recherche français ou étrangers, des laboratoires publics ou privés. 


\title{
Typed binary trees and generalized dendrifom algebras
}

\author{
Loïc Foissy \\ Fédération de Recherche Mathématique du Nord Pas de Calais FR 2956, \\ Laboratoire de Mathématiques Pures et Appliquées Joseph Liouville, \\ Université du Littoral Côte d'Opale* \\ Email: foissy@univ-littoral.fr
}

\begin{abstract}
We here both unify and generalize nonassociative structures on typed binary trees, that is to say plane binary trees which edges are decorated by elements of a set $\Omega$. We prove that we obtain such a structure, called a $\Omega$-dendriform structure, if $\Omega$ has four products satisfying certain axioms (EDS axioms), including the axioms of a diassociative semigroup. This includes matching dendriform algebras introduced by Gao, Guo and Zhang and family dendriform algebras associated to a semigroup introduced by Zhang, Gao and Manchon, and of course dendriform algebras when $\Omega$ is reduced to a single element. We also give examples of EDS, including all the EDS of cardinality two; a combinatorial description of the products of such a structure on typed binary trees, but also on words; a study of the Koszul dual of the associated operads; and considerations on the existence of a coproduct, in order to obtain dendriform bialgebras.
\end{abstract}

Keywords. Dendriform algebra; diassociative semigroup; plane binary trees; shuffle product.

AMS classification. 16T30; 05C05; 18D50.

\section{Contents}

\begin{tabular}{|lll}
\hline 1 & (Extended) diassociative semigroups & 5
\end{tabular}

1.1 Diassociative semigroups $\ldots \ldots \ldots \ldots \ldots \ldots \ldots$

1.2 Extended diassociative semigroups $\ldots \ldots \ldots \ldots \ldots$

1.3 Nondegenerate extended diassociative semigroups $\ldots \ldots \ldots \ldots$

$1.4 \quad$ Extended diassociative semigroups of cardinality two . . . . . . . . . . . . . . . . 13

$\begin{array}{llr}2 & \Omega \text {-dendriform algebras } & 16\end{array}$

2.1 Definition and example . . . . . . . . . . . . . . . . . . . . . . . . . . 16

$2.2 \quad$ Structures on typed binary trees $\ldots \ldots \ldots \ldots \ldots$. . . . . . . . . . . . 16

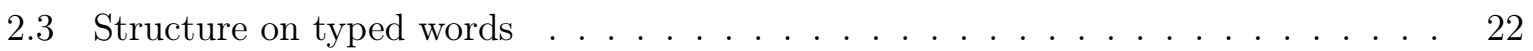

$2.4 \quad$ From $\Omega$-dendrifrom algebras to dendriform algebras . . . . . . . . . . . . . . . . . 25

$\begin{array}{lll}3 & \text { Operad of } \Omega \text {-dendriform algebras } & 28\end{array}$

$3.1 \quad$ Combinatorial description of the operad $\ldots \ldots \ldots \ldots \ldots \ldots$

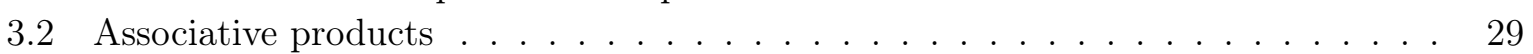

3.3 Dendriform products $\ldots \ldots \ldots \ldots \ldots$

3.4 Koszul dual . . . . . . . . . . . . . . . . . . . . . . . . . . . . . . 34

${ }^{*}$ Centre Universitaire de la Mi-Voix, 50, rue Ferdinand Buisson, CS 80699, 62228 Calais Cedex, France 
4 Combinatorial description of the products 37

4.1 On typed trees . . . . . . . . . . . . . . . . . . . . . . . . . . . . 37

4.2 On typed words . . . . . . . . . . . . . . . . . . . . . . . . . . . . 41

\begin{tabular}{|lll}
5 & Hopf algebraic structure & $\mathbf{4 1}$
\end{tabular}

$5.1 \quad$ Existence of dendriform bialgebraic structures . . . . . . . . . . . . . . . . . . . . 41

5.2 Combinatorial description of the coproducts on typed trees . . . . . . . . . . . . 42

5.3 Combinatorial description of the coproducts on typed words . . . . . . . . . . . . 44

\begin{tabular}{lr}
\hline References & 45 \\
\hline
\end{tabular}

\section{Introduction}

Dendriform algebras are associative algebras with an associativity splitting, that is to say their associative product can be written as a sum of two products $\prec$ and $>$, with the following axioms:

$$
\begin{aligned}
(x<y) & <z=x<(y<z+y>z), \\
(x>y) & <z=x>(y<z), \\
(x<y+x>y) & >z=x>(y>z) .
\end{aligned}
$$

Note that summing these three relations proves that, indeed, $<+>$ is associative. Classical examples of dendriform algebras are given by shuffle algebras, based on words, as noticed by Schützenberger in [13, which justifies the terminology of noncommutative shuffle algebras used for example in [6]. Free dendriform algebras were first described by Loday and Ronco [11] and studied in [1]: the free dendriform algebra on one generator is based on plane binary trees, and its two products $\prec$ and $>$ are inductively defined using the decomposition of any plane binary tree (except the unit ।) into a left and a right plane binary tree. For example, here are plane binary trees with $k=2,3$ or 4 leaves:
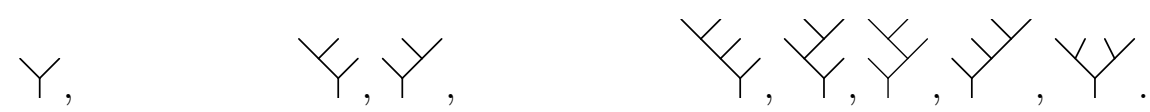

Here are examples of products on plane binary trees:
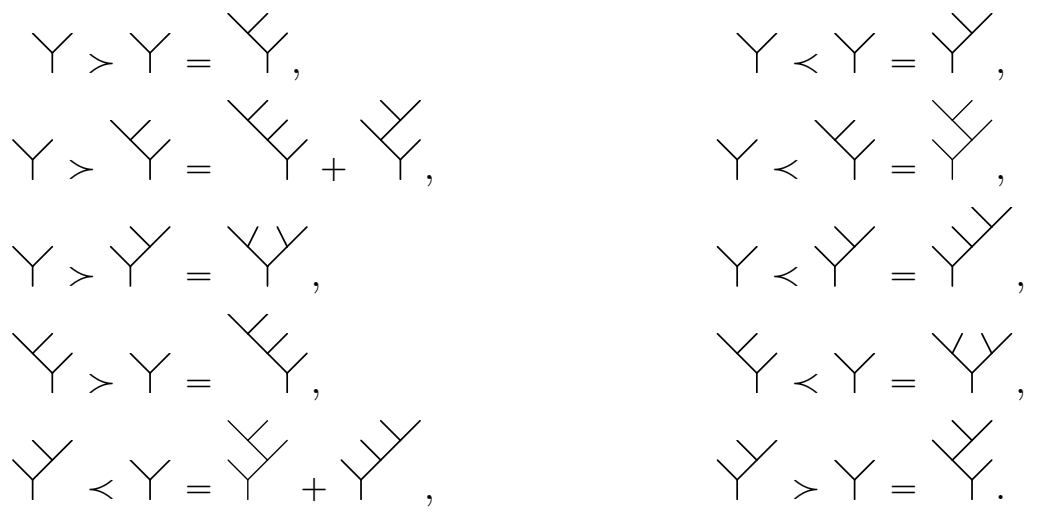

Recently, a new interest in typed trees were developed in the seminal work of Bruned, Hairer and Zambotti on stochastic PDEs [3]. Given a nonempty set $\Omega$, called the set of types, a $\Omega$-typed tree is a tree with a map from this set of edges to $\Omega$ : they are related to a generalization of pre-Lie algebras [5]. Similarly, several generalizations of dendriform algebras were recently introduced, where plane binary trees are replaced by typed plane binary trees. 
- Firstly, if $\Omega$ is a set, a $\Omega$-matching dendriform algebras [7] is a vector space $A$ with products $\prec_{\alpha},>_{\alpha}$, where $\alpha \in \Omega$, such that:

$$
\begin{aligned}
\left(x \prec_{\alpha} y\right) \prec_{\beta} z & =x<_{\alpha}\left(y<_{\beta} z\right)+x \prec_{\beta}\left(y>_{\alpha} z\right), \\
\left(x>_{\alpha} y\right) \prec_{\beta} z & =x>_{\alpha}\left(y<_{\beta} z\right), \\
\left(x \prec_{\beta} y\right)>_{\alpha} z+\left(x>_{\alpha} y\right)>_{\beta} z & =x>_{\alpha}\left(y>_{\beta} z\right) .
\end{aligned}
$$

- Secondly, if $(\Omega, *)$ is a semigroup, a $\Omega$-family dendriform algebra 14 is a vector space $A$ with products $<_{\alpha},>_{\alpha}$, where $\alpha \in \Omega$, such that:

$$
\begin{aligned}
\left(x \prec_{\alpha} y\right) \prec_{\beta} z & =x<_{\alpha * \beta}\left(y<_{\beta} z+y>_{\alpha} z\right), \\
\left(x>_{\alpha} y\right)<_{\beta} z & =x>_{\alpha}\left(y<_{\beta} z\right), \\
\left(x<_{\beta} y+x>_{\alpha} y\right)>_{\alpha * \beta} z & =x>_{\alpha}\left(y>_{\beta} z\right) .
\end{aligned}
$$

In both cases, it was proved that the free object on one generator is based on plane $\Omega$-typed binary trees, with products inductively defined in a similar way as the Loday-Ronco's construction. A plane $\Omega$-typed binary tree is a plane binary tree given a map from the set of its internal edges to $\Omega$. We shall denote them in the following way:

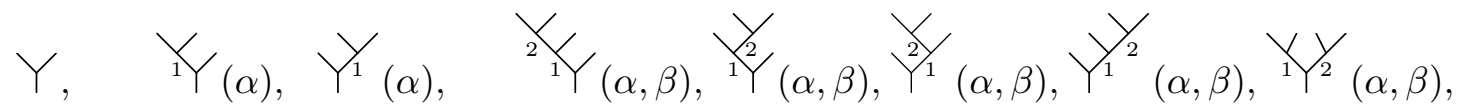

where $\alpha, \beta \in \Omega$. In all cases, the type of the internal edge 1 is $\alpha$ and the type of the internal edge 2 is $\beta$. Here are examples of products in the $\Omega$-matching case:

$$
\begin{aligned}
& Y>_{\alpha} Y(\alpha)={ }^{Y}(\alpha), \\
& Y_{>_{\alpha}}{ }^{Y} Y_{(\beta)}={ }^{{ }^{Y}}{ }^{Y}(\beta, \alpha)+{ }^{12}(\alpha, \beta), \\
& Y>_{\alpha} Y^{1}(\beta)={ }^{1} Y^{2}{ }_{(\alpha, \beta),} \\
& { }^{Y}{ }_{(\alpha)>_{\beta}} Y={ }^{2} Y(\beta, \alpha) \text {, } \\
& Y_{1}{ }_{(\alpha)<_{\beta}} Y=Y^{2 / 1}(\alpha, \beta)+Y_{1}{ }^{2}(\beta, \alpha),
\end{aligned}
$$

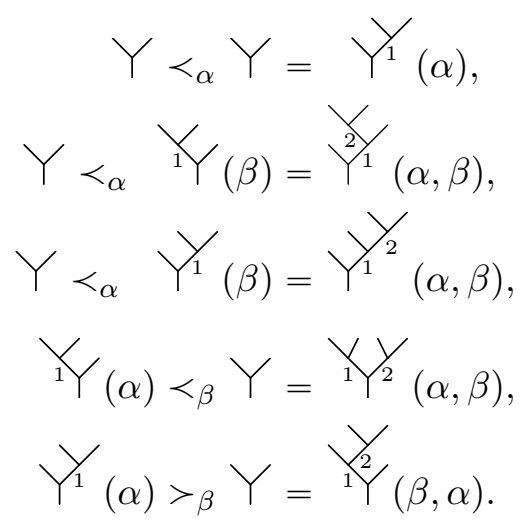

Here are examples of products in the $\Omega$-family case:

$$
\begin{aligned}
& Y>_{\alpha} Y(\alpha)={ }^{Y} Y(\alpha), \\
& Y>_{\alpha} Y_{1}(\beta)={ }^{2}{ }_{1}(\alpha * \beta, \alpha)+{ }^{1} Y_{(\alpha * \beta, \beta)}, \\
& Y>_{\alpha} Y^{1}(\beta)={ }^{1} Y^{2}{ }^{(\alpha, \beta)}, \\
& { }^{Y}(\alpha)>_{\beta} Y={ }^{2} Y(\beta, \alpha) \text {, } \\
& Y^{1}{ }_{(\alpha)<_{\beta}} Y=Y^{2} / 1^{\prime}(\alpha * \beta, \beta)+Y^{Y / 2}(\alpha * \beta, \alpha),
\end{aligned}
$$

$$
\begin{aligned}
& Y \prec_{\alpha} Y=Y_{1}(\alpha) \\
& Y<_{\alpha} Y_{(\beta)}=Y^{2 / 1}(\alpha, \beta) \text {, } \\
& Y \prec_{\alpha} Y_{1}{ }_{(\beta)}=Y_{1}{ }^{2}(\alpha, \beta), \\
& { }^{1} Y(\alpha)<_{\beta} Y={ }^{1} Y{ }^{2}(\alpha, \beta) \text {, } \\
& Y_{1}(\alpha)>_{\beta} Y=Y^{1 / 2}(\beta, \alpha) \text {. }
\end{aligned}
$$

Our aim in this article is to give both a unification and a generalization of the extended dendriform structures. We start with a set of types $\Omega$, given four operations $\leftarrow, \rightarrow, \triangleleft, \triangleright$. A 
$\Omega$-dendriform algebra is a vector space $A$ with products $<_{\alpha},>_{\alpha}$, where $\alpha \in \Omega$, such that:

$$
\begin{aligned}
\left(x \prec_{\alpha} y\right)<_{\beta} z & =x \prec_{\alpha \leftarrow \beta}\left(y \prec_{\alpha \triangleleft \beta} z\right)+x \prec_{\alpha \rightarrow \beta}\left(y>_{\alpha \triangleright \beta} z\right), \\
x>_{\alpha}\left(<_{\beta} z\right) & =\left(x>_{\alpha} y\right)<_{\beta} z, \\
x>_{\alpha}\left(y>_{\beta} z\right) & =\left(x>_{\alpha \triangleright \beta} y\right)>_{\alpha \rightarrow \beta} z+\left(x \prec_{\alpha \triangleleft \beta} y\right)>_{\alpha \leftarrow \beta} z .
\end{aligned}
$$

We recover the notion of $\Omega$-matching dendriform algebra taking:

$$
\begin{aligned}
& \forall \alpha, \beta \in \Omega, \\
& \alpha \leftarrow \beta=\alpha, \\
& \alpha \rightarrow \beta=\beta, \\
& \alpha \triangleleft \beta=\beta, \\
& \alpha \triangleright \beta=\alpha ;
\end{aligned}
$$

and we recover the notion of $\Omega$-family dendriform algebra taking, for any $\alpha, \beta \in \Omega$ :

$$
\begin{array}{rlrl}
\forall \alpha, \beta \in \Omega, & \alpha \leftarrow \beta=\alpha * \beta, & \alpha \rightarrow \beta & =\alpha * \beta, \\
\alpha \triangleleft \beta=\beta, & \alpha \triangleright \beta=\alpha ;
\end{array}
$$

We prove in Proposition 15 that the free $\Omega$-dendriform algebra on one generator is based on plane $\Omega$-typed binary trees, with an inductive definition of the products $<_{\alpha}$ and $>_{\beta}$, if, and only if, the four operations of $\Omega$ satisfy a bunch of 15 axioms, see Definitions 1 and 2 a similar result is proved for words in Proposition 17, giving typed versions of shuffle algebras. Such a structure on $\Omega$ will be called an extended diassociative semigroup (briefly, EDS); in particular, the first five axioms only involve the two operations $\leftarrow$ and $\rightarrow$ :

$$
\begin{aligned}
& (\alpha \leftarrow \beta) \leftarrow \gamma=\alpha \leftarrow(\beta \leftarrow \gamma)=\alpha \leftarrow(\beta \rightarrow \gamma), \\
& (\alpha \rightarrow \beta) \leftarrow \gamma=\alpha \rightarrow(\beta \leftarrow \gamma), \\
& (\alpha \rightarrow \beta) \rightarrow \gamma=(\alpha \leftarrow \beta) \rightarrow \gamma=\alpha \rightarrow(\beta \rightarrow \gamma) .
\end{aligned}
$$

These axioms are ruled by the operad on diassociative algebras, which suggested our terminology. A noticeable fact is that this operad is the Koszul dual of the dendriform operad. Examples of EDS include the ones, denoted by $\operatorname{EDS}(\Omega)$, giving matching dendriform algebras; the ones, denoted by $\operatorname{EDS}(\Omega, *)$, giving family dendriform algebras; and lots more. For example, if $\Omega$ is of cardinality two, we found $24 \mathrm{EDS}$, including $\operatorname{EDS}(\Omega)$ and 5 coming from associative semigroups.

We prove that any $\Omega$-dendriform algebra $A$ gives a dendriform algebra structure on the space $\mathbb{K} \Omega \otimes A$ (Proposition 18 ): this was already known in the case of $\Omega$-matching dendriform algebras 8]. The converse implication is true under a condition of nondegeneracy of the EDS $\Omega$.

The description of free $\Omega$-dendriform algebras induces a combinatorial description of their operad. When $\Omega$ is finite, this is a quadratic finitely generated operad, which Koszul dual is described in Proposition 27. This operad is not always Koszul, and we produce a necessary condition ( $\Omega$ should be weakly nondegenerate, Definition 28$)$ and a sufficient condition on it $(\Omega$ should be nondegenerate, see Definition 4 for the associated operad to be Koszul. For example, $\operatorname{EDS}(\Omega)$ is nondegenerate; if $(\Omega, *)$ is a finite associative semigroup, then $\operatorname{EDS}(\Omega, *)$ is nondegenerate if, and only $(\Omega, *)$ is a group.

We also give a study of these objects, from a Hopf-algebraic and a combinatorial point of view. In particular, we give a description of the products on trees and on words in Propositions 33 and 36, generalizing in the latter case the usual half-shuffle products. Shuffle algebras and the Loday-Ronco algebra are known to be Hopf algebras; this is not always true for $\Omega$-dendriform algebras, as described in Proposition 38 . If $\Omega$ is nondegenerate, then such a structure exists on trees and on words (Propositions 39 and 40 , which is combinatorially described in Propositions 42 and 43 . These coproducts generalize the Loday-Ronco coproduct on trees and the deconcatenation coproducts on words. 
This paper is organized as follows: the first section is devoted to the study of EDS. We give examples based on (diassociative) monoids, and semidirect products of groups. We also introduce nondegenerate EDS, with a reformulation of their axioms due to a transformation of the four defining operations into four other ones; this allows to associate to any group $G$ a nondegenerate EDS EDS* $(G)$ (Proposition 9). We prove some results on particular families of EDS: for example, we give in Proposition 10 all nondegenerate finite $\Omega$ such that if $\alpha$ and $\beta \in \Omega$,

$$
\alpha \leftarrow \beta=\beta \rightarrow \alpha=\alpha .
$$

We also give in this section a complete classification of EDS of cardinality 2 (24 objects, which 4 are nondegenerate).

The second section is devoted to the definition of $\Omega$-dendriform algebras and to the structure on trees and words, when $\Omega$ is an EDS. The operadic aspects are considered in the next section, with in particular the results on the Koszulity; we also study the associative products and the dendriform products (that is to say, morphisms from the operad of associative algebras and from the the operad of dendriform algebras) in $\Omega$-dendriform algebras in particular cases of $\Omega$.

We finally give a combinatorial description of the products in Section 5 and the last section is devoted to the existence of the coproducts and their combinatorial descriptions.

Acknowledgements. The author is grateful to Professor Xing Gao, his team and Lanzhou University for their warm hospitality.

Notations $1 . \mathbb{K}$ is a commutative field. All the vector spaces in this text will be taken over $\mathbb{K}$. If $S$ is a set, we denote by $\mathbb{K} S$ the vector space generated by $S$.

\section{1 (Extended) diassociative semigroups}

\subsection{Diassociative semigroups}

Definition 1. A diassociative semigroup is a family $(\Omega, \leftarrow, \rightarrow)$, where $\Omega$ is a set and $\leftarrow, \rightarrow$ : $\Omega \times \Omega \longrightarrow \Omega$ are maps such that, for any $\alpha, \beta, \gamma \in \Omega$ :

$$
\begin{aligned}
& (\alpha \leftarrow \beta) \leftarrow \gamma=\alpha \leftarrow(\beta \leftarrow \gamma)=\alpha \leftarrow(\beta \rightarrow \gamma), \\
& (\alpha \rightarrow \beta) \leftarrow \gamma=\alpha \rightarrow(\beta \leftarrow \gamma), \\
& (\alpha \rightarrow \beta) \rightarrow \gamma=(\alpha \leftarrow \beta) \rightarrow \gamma=\alpha \rightarrow(\beta \rightarrow \gamma) .
\end{aligned}
$$

Example 1. 1. If $(\Omega, \star)$ is an associative semigroup, then $(\Omega, \star, \star)$ is a diassociative semigroup.

2. Let $\Omega$ be a set. We put:

$$
\forall \alpha, \beta \in \Omega, \quad \alpha \leftarrow \beta=\alpha, \quad \alpha \rightarrow \beta=\beta .
$$

Then $(\Omega, \leftarrow, \rightarrow)$ is a diassociative semigroup, denoted by $\mathbf{D S}(\Omega)$.

3. Let $\Omega=(\Omega, \leftarrow, \rightarrow)$ be a diassociative semigroup. We define two new operations on $\Omega$ by:

$$
\forall \alpha, \beta \in \Omega, \quad \alpha \leftarrow^{o p} \beta=\beta \rightarrow \alpha, \quad \alpha \rightarrow^{o p} \beta=\beta \leftarrow \alpha .
$$

This defines a new diassociative semigroup $\Omega^{o p}=\left(\Omega, \leftarrow^{o p}, \rightarrow^{o p}\right)$. We shall say that $\Omega$ is commutative if $\Omega=\Omega^{o p}$, that is to say:

$$
\forall \alpha, \beta \in \Omega, \quad \alpha \rightarrow \beta=\beta \leftarrow \alpha .
$$

In other words, a commutative diassociative semigroup is a pair $(\Omega, \leftarrow)$ such that, for any $\alpha, \beta, \gamma \in \Omega$ :

$$
(\alpha \leftarrow \beta) \leftarrow \gamma=\alpha \leftarrow(\beta \leftarrow \gamma)=(\alpha \leftarrow \gamma) \leftarrow \beta .
$$




\subsection{Extended diassociative semigroups}

Definition 2. An extended diassociative semigroup (briefly, EDS) is a family $(\Omega, \leftarrow, \rightarrow, \triangleleft, \triangleright)$, where $\Omega$ is a set and $\leftarrow, \rightarrow, \triangleleft, \triangleright: \Omega \times \Omega \longrightarrow \Omega$ are maps such that:

1. $(\Omega, \leftarrow, \rightarrow)$ is a diassociative semigroup.

2. For any $\alpha, \beta, \gamma \in \Omega$ :

$$
\begin{aligned}
\alpha \triangleright(\beta \leftarrow \gamma) & =\alpha \triangleright \beta, \\
(\alpha \rightarrow \beta) \triangleleft \gamma & =\beta \triangleleft \gamma, \\
(\alpha \triangleleft \beta) \leftarrow((\alpha \leftarrow \beta) \triangleleft \gamma) & =\alpha \triangleleft(\beta \leftarrow \gamma), \\
(\alpha \triangleleft \beta) \triangleleft((\alpha \leftarrow \beta) \triangleleft \gamma) & =\beta \triangleleft \gamma, \\
(\alpha \triangleleft \beta) \rightarrow((\alpha \leftarrow \beta) \triangleleft \gamma) & =\alpha \triangleleft(\beta \rightarrow \gamma), \\
(\alpha \triangleleft \beta) \triangleright((\alpha \leftarrow \beta) \triangleleft \gamma) & =\beta \triangleright \gamma, \\
(\alpha \triangleright(\beta \rightarrow \gamma)) \leftarrow(\beta \triangleright \gamma) & =(\alpha \leftarrow \beta) \triangleright \gamma, \\
(\alpha \triangleright(\beta \rightarrow \gamma)) \triangleleft(\beta \triangleright \gamma) & =\alpha \triangleleft \beta, \\
(\alpha \triangleright(\beta \rightarrow \gamma)) \rightarrow(\beta \triangleright \gamma) & =(\alpha \rightarrow \beta) \triangleright \gamma, \\
(\alpha \triangleright(\beta \rightarrow \gamma)) \triangleright(\beta \triangleright \gamma) & =\alpha \triangleright \beta .
\end{aligned}
$$

Example 2. 1. Let $\Omega=(\Omega, \leftarrow, \rightarrow)$ be a diassociative semigroup. We define two products on $\Omega$ by:

$$
\forall \alpha, \beta \in \Omega, \quad \alpha \triangleleft \beta=\beta, \quad \alpha \triangleright \beta=\alpha .
$$

Then $(\Omega, \leftarrow, \rightarrow, \triangleleft, \triangleright)$ is an EDS, denoted by $\operatorname{EDS}(\Omega, \leftarrow, \rightarrow)$. When $(\Omega, \leftarrow, \rightarrow)=\operatorname{DS}(\Omega)$, we shall simply write $\mathbf{E D S}(\Omega)$.

2. Let $\Omega=(\Omega, \star)$ be an associative semigroup. If $\triangleleft$ and $\triangleright$ are products on $\Omega$, then $(\Omega, \star, \star, \triangleleft, \triangleright)$ is an EDS if, and only if, for any $\alpha, \beta, \gamma \in \Omega$ :

$$
\begin{gathered}
\alpha \triangleright(\beta \star \gamma)=\alpha \triangleright \beta, \\
(\alpha \star \beta) \triangleleft \gamma=\beta \triangleleft \gamma, \\
(\alpha \triangleleft \beta) \star(\beta \triangleleft \gamma)=\alpha \triangleleft(\beta \star \gamma), \\
(\alpha \triangleleft \beta) \triangleleft(\beta \triangleleft \gamma)=\beta \triangleleft \gamma, \\
(\alpha \triangleleft \beta) \triangleright(\beta \triangleleft \gamma)=\beta \triangleright \gamma, \\
(\alpha \triangleright \beta) \star(\beta \triangleright \gamma)=(\alpha \star \beta) \triangleright \gamma, \\
(\alpha \triangleright \beta) \triangleleft(\beta \triangleright \gamma)=\alpha \triangleleft \beta, \\
(\alpha \triangleright \beta) \triangleright(\beta \triangleright \gamma)=\alpha \triangleright \beta .
\end{gathered}
$$

3. Let $\Omega$ be a set and let $\operatorname{DS}(\Omega)$ be the diassociative semigroup attached to $\Omega$. If $\triangleleft$ and $\triangleright$ are products on $\Omega$, then $(\Omega, \leftarrow, \rightarrow, \triangleleft, \triangleright)$ is an EDS if, and only if, for any $\alpha, \beta, \gamma \in \Omega$ :

$$
\begin{aligned}
& (\alpha \triangleleft \beta) \triangleleft(\alpha \triangleleft \gamma)=\beta \triangleleft \gamma, \\
& (\alpha \triangleleft \beta) \triangleright(\alpha \triangleleft \gamma)=\beta \triangleright \gamma, \\
& (\alpha \triangleright \gamma) \triangleleft(\beta \triangleright \gamma)=\alpha \triangleleft \beta, \\
& (\alpha \triangleright \gamma) \triangleright(\beta \triangleright \gamma)=\alpha \triangleright \beta .
\end{aligned}
$$


4. Let $\Omega=(\Omega, \leftarrow, \rightarrow)$ be a diassociative semigroup, and let $\phi_{\triangleleft}, \phi_{\triangleright}: \Omega \longrightarrow \Omega$ be two maps. We define two products on $\Omega$ by:

$$
\forall \alpha, \beta \in \Omega, \quad \alpha \triangleleft \beta=\phi_{\triangleleft}(\beta), \quad \alpha \triangleright \beta=\phi_{\triangleright}(\alpha) .
$$

Then $(\Omega, \leftarrow, \rightarrow, \triangleleft, \triangleright)$ is an EDS if, and only if:

$$
\begin{aligned}
& \phi_{\triangleleft}=\phi_{\triangleleft} \circ \phi_{\triangleleft}=\phi_{\triangleleft} \circ \phi_{\triangleright}, \\
& \phi_{\triangleright}=\phi_{\triangleright} \circ \phi_{\triangleleft}=\phi_{\triangleright} \circ \phi_{\triangleright},
\end{aligned}
$$

and, for any $\alpha, \beta \in \Omega$ :

$$
\begin{aligned}
& \phi_{\triangleleft}(\alpha \leftarrow \beta)=\phi_{\triangleleft}(\alpha) \leftarrow \phi_{\triangleleft}(\beta), \\
& \phi_{\triangleleft}(\alpha \rightarrow \beta)=\phi_{\triangleleft}(\alpha) \rightarrow \phi_{\triangleleft}(\beta), \\
& \phi_{\triangleright}(\alpha \leftarrow \beta)=\phi_{\triangleright}(\alpha) \leftarrow \phi_{\triangleright}(\beta), \\
& \phi_{\triangleright}(\alpha \rightarrow \beta)=\phi_{\triangleright}(\alpha) \rightarrow \phi_{\triangleright}(\beta),
\end{aligned}
$$

that is to say $\phi_{\triangleleft}$ and $\phi_{\triangleright}$ are diassociative semigroup morphisms. If so, the obtained EDS is denoted by $\operatorname{EDS}\left(\Omega, \leftarrow, \rightarrow, \phi_{\triangleleft}, \phi_{\triangleright}\right)$. In particular,

$$
\operatorname{EDS}\left(\Omega, \leftarrow, \rightarrow, I d_{\Omega}, I d_{\Omega}\right)=\operatorname{EDS}(\Omega, \leftarrow, \rightarrow) .
$$

5. Let $(\Omega, \leftarrow, \rightarrow, \triangleleft, \triangleright)$ be an EDS. We define four new products on $\Omega$ by:

$$
\begin{aligned}
& \forall \alpha, \beta \in \Omega \text {, } \\
& \alpha \leftarrow^{o p} \beta=\beta \rightarrow \alpha, \\
& \alpha \rightarrow^{o p} \beta=\beta \leftarrow \alpha, \\
& \alpha \triangleleft^{o p} \beta=\beta \triangleright \alpha, \\
& \alpha \triangleright^{o p} \beta=\beta \triangleleft \alpha \text {. }
\end{aligned}
$$

This defines a new diassociative semigroup $\Omega^{o p}=\left(\Omega, \leftarrow^{o p}, \rightarrow^{o p}, \triangleleft^{o p}, \triangleright^{o p}\right)$. We shall say that $\Omega$ is commutative if $\Omega=\Omega^{o p}$, that is to say, for any $\alpha, \beta, \gamma \in \Omega$ :

$$
\alpha \rightarrow \beta=\beta \leftarrow \alpha, \quad \alpha \triangleright \beta=\beta \triangleleft \alpha .
$$

In the case of groups, we find semidirect products:

Proposition 3. Let $(\Omega, \star, \star, \triangleleft, \triangleright)$ be an EDS, such that $(\Omega, \star)$ is a group. There exist three subgroups $H, K_{\triangleleft}$ and $K_{\triangleright}$ of $\Omega$ such that

$$
G=K \rtimes H_{\triangleleft}=K \rtimes H_{\triangleright} .
$$

Moreover, for any $\alpha, \beta \in \Omega, \alpha \triangleleft \beta$ is the canonical projection of $\beta$ on $H_{\triangleleft}$ and $\alpha \triangleright \beta$ is the canonical projection of $\alpha$ on $H_{\triangleright}$.

Proof. Let $\alpha, \beta, \beta^{\prime} \in \Omega$. As $\Omega$ is a group, there exists $\gamma \in \Omega$ such that $\beta \star \gamma=\beta^{\prime}$. By (14), $\alpha \triangleright \beta^{\prime}=\alpha \triangleright \beta$. Hence, there exists a map $\phi_{\triangleright}: \Omega \longrightarrow \Omega$ such that:

$$
\forall \alpha, \beta \in \Omega, \quad \phi_{\triangleright}(\alpha)=\alpha \triangleright \beta .
$$

Similarly, we deduce from 15 the existence of a map $\phi_{\triangleleft}: \Omega \longrightarrow \Omega$ such that

$$
\forall \alpha, \beta \in \Omega, \quad \quad \phi_{\triangleleft}(\beta)=\alpha \triangleleft \beta .
$$

By (28)-(31), $\phi_{\triangleleft}$ and $\phi_{\triangleright}$ are group morphisms. Let us denote by $K_{\triangleleft}$ and $K_{\triangleright}$ their respective kernels, and by $H_{\triangleleft}$ and $H_{\triangleright}$ their respective images. By (26) and 27), $\phi_{\triangleleft}^{2}=\phi_{\triangleleft}$ and $\phi_{\triangleright}^{2}=\phi_{\triangleright}$, so:

$$
G=K_{\triangleleft} \rtimes H_{\triangleleft}=K_{\triangleright} \rtimes H_{\triangleright} .
$$

Moreover, $\phi_{\triangleleft}$ and $\phi_{\triangleright}$ are the canonical projection on, respectively, $H_{\triangleleft}$ and $H_{\triangleright}$.

Let $\alpha \in K_{\triangleleft}$. Then $\phi_{\triangleleft}(\alpha)=e_{G}$, and, by (27):

$$
\phi_{\triangleright}(\alpha)=\phi_{\triangleright} \circ \phi_{\triangleleft}(\alpha)=\phi_{\triangleright}\left(e_{G}\right)=e_{G},
$$

so $\alpha \in K_{\triangleright}$. By symmetry, $K_{\triangleleft}=K_{\triangleright}=K$. 
Remark 1. Conversely, if (32) is satisfied, the canonical projections $\phi_{\triangleleft}$ and $\phi_{\triangleright}$ satisfy (26)-(31), so we obtain an EDS.

\subsection{Nondegenerate extended diassociative semigroups}

Definition 4. Let $\Omega=(\Omega, \leftarrow, \rightarrow, \triangleleft, \triangleright)$ be an EDS. We define the following maps:

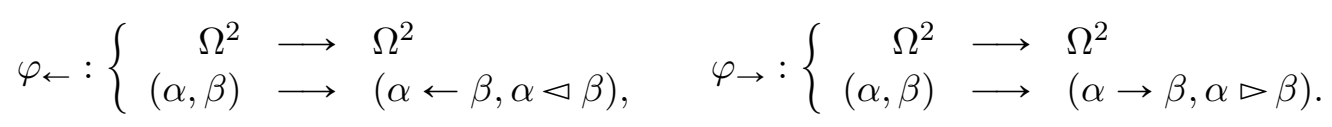

We shall say that $\Omega$ is nondegenerate if $\varphi_{\leftarrow}$ and $\varphi_{\rightarrow}$ are bijective.

The axioms of EDS can be entirely given with the help of the maps $\varphi_{\leftarrow}$ and $\varphi_{\rightarrow}$ :

Lemma 5. Let $\Omega=(\Omega, \leftarrow, \rightarrow, \triangleleft, \triangleright)$ be a set with four products. We define $\varphi_{\leftarrow}$ and $\varphi_{\rightarrow}$ by (33), and we put:

$$
\tau:\left\{\begin{array}{rll}
\Omega^{2} & \longrightarrow \Omega^{2} \\
(\alpha, \beta) & \longrightarrow(\beta, \alpha) .
\end{array}\right.
$$

Then $\Omega$ is an EDS if, and only if:

$$
\begin{aligned}
& (\tau \otimes I d) \circ\left(I d \otimes \varphi_{\leftarrow}\right) \circ(\tau \otimes I d) \circ\left(\varphi_{\rightarrow} \otimes I d\right)=\left(\varphi_{\rightarrow} \otimes I d\right) \circ\left(I d \otimes \varphi_{\leftarrow}\right), \\
& \left(I d \otimes \varphi_{\leftarrow}\right) \circ(\tau \otimes I d) \circ\left(I d \otimes \varphi_{\leftarrow}\right) \otimes(\tau \otimes I d) \circ\left(\varphi_{\leftarrow} \otimes I d\right)=\left(\varphi_{\leftarrow} \otimes I d\right) \circ\left(I d \otimes \varphi_{\leftarrow}\right), \\
& \left(I d \otimes \varphi_{\rightarrow}\right) \circ(\tau \otimes I d) \circ\left(I d \otimes \varphi_{\leftarrow}\right) \circ(\tau \otimes I d) \circ\left(\varphi_{\leftarrow} \otimes I d\right)=\left(\varphi_{\leftarrow} \otimes I d\right) \circ\left(I d \otimes \varphi_{\rightarrow}\right), \\
& \left(I d \otimes \varphi_{\leftarrow}\right) \circ\left(\varphi_{\rightarrow} \otimes I d\right) \circ\left(I d \otimes \varphi_{\rightarrow}\right)=\left(\varphi_{\rightarrow} \otimes I d\right) \circ(I d \otimes \tau) \circ\left(\varphi_{\leftarrow} \otimes I d\right), \\
& \left(I d \otimes \varphi_{\rightarrow}\right) \circ\left(\varphi_{\rightarrow} \otimes I d\right) \circ\left(I d \otimes \varphi_{\rightarrow}\right)=\left(\varphi_{\rightarrow} \otimes I d\right) \circ(I d \otimes \tau) \circ\left(\varphi_{\rightarrow} \otimes I d\right) .
\end{aligned}
$$

Proof. Direct computations prove that (34) is equivalent to (2), (4) and (5); (35) to one of the equalities of (1), (6) and (7); (36) to the other equality of (1), (8) and (9); (37) to one of the equalities of (3), (10) and (11); (38) to the other equality of (3), (12) and (13).

Proposition 6. Let $\Omega=(\Omega, \leftarrow, \rightarrow, \triangleleft, \triangleright)$ be a nondegenerate EDS. We define four products $\curvearrowleft$, $\supset$,, and $\bullet$ on $\Omega$ by:

$$
\forall \alpha, \beta \in \Omega, \quad \varphi_{\leftarrow}^{-1}(\alpha, \beta)=(\alpha \curvearrowleft \beta, \alpha \triangleleft \beta), \quad \varphi_{\rightarrow}^{-1}(\alpha, \beta)=(\beta \frown \beta, \beta \bullet \alpha) .
$$

Then, for any $\alpha, \beta, \gamma \in \Omega$ :

$$
\begin{aligned}
& (\alpha \triangleleft \beta) \curvearrowleft \gamma=\alpha \triangleleft(\beta \curvearrowleft \gamma) \\
& \alpha \triangleleft(\beta \bullet \gamma)=(\alpha \triangleleft \beta) \triangleleft \gamma, \quad(\alpha \bullet \beta) \bullet \gamma=\alpha \bullet(\beta \bullet \gamma), \\
& \alpha \bullet(\beta \curvearrowleft \gamma)=\alpha \bullet \beta, \quad(\alpha \frown \beta) \triangleleft \gamma=\beta \triangleleft \gamma, \\
& (\alpha \curvearrowleft(\beta \triangleleft \gamma)) \curvearrowleft(\beta \curvearrowleft \gamma)=\alpha \curvearrowleft \beta, \quad(\alpha \frown \beta) \frown((\alpha \bullet \beta) \frown \gamma)=\beta \frown \gamma, \\
& (\alpha \curvearrowleft(\beta \triangleleft \gamma)) \triangleleft(\beta \curvearrowleft \gamma)=(\alpha \triangleleft \beta) \curvearrowleft \gamma, \quad(\alpha \frown \beta) \bullet((\alpha \bullet \beta) \neg \gamma)=\alpha \frown(\beta \triangleright \gamma), \\
& (\alpha \curvearrowleft(\beta \frown \gamma)) \curvearrowleft(\beta \neg \gamma)=\alpha \curvearrowleft \gamma, \quad(\alpha \triangleleft \beta) \frown((\alpha \curvearrowleft \beta) \frown \gamma)=\alpha \frown \gamma, \\
& (\alpha \curvearrowleft(\beta \frown \gamma)) \triangleleft(\beta \bullet \gamma)=\beta \bullet(\alpha \triangleleft \gamma), \quad(\alpha \triangleleft \beta) \bullet((\alpha \curvearrowleft \beta) \frown \gamma)=(\alpha \bullet \gamma) \triangleleft \beta, \\
& (\alpha \curvearrowleft \beta) \bullet \gamma=(\alpha \bullet \gamma) \curvearrowleft \beta, \quad \beta \frown(\alpha \triangleleft \gamma)=\alpha \triangleleft(\beta \frown \gamma) \text {. }
\end{aligned}
$$

In particular, $\bullet$ and $\hookrightarrow$ are associative. 
Proof. By (34):

$$
\left(\varphi_{\leftarrow}^{-1} \otimes I d\right) \circ(\tau \otimes I d) \circ\left(I d \otimes \varphi_{\leftarrow}^{-1}\right) \circ(\tau \otimes I d)=\left(I d \otimes \varphi_{\leftarrow}^{-1}\right) \circ\left(\varphi_{\rightarrow}^{-1} \otimes I d\right) .
$$

When applied to $(\beta, \alpha, \gamma)$, we obtain:

$$
(\alpha \triangleright(\beta \curvearrowleft \gamma),(\alpha \frown \beta) \curvearrowleft \gamma,(\alpha \frown \beta) \triangleleft \gamma)=(\alpha \triangleright \gamma, \alpha \frown(\beta \curvearrowleft \gamma), \beta \triangleleft \gamma)
$$

The other identities are proved in the same way, from (35)- 38 .

We now explore two families of nondegenerate EDS.

Lemma 7. Let $(\Omega, \star)$ be an associative semigroup. The following conditions are equivalent:

1. $\operatorname{EDS}(\Omega, \star, \star)$ is nondegenerate.

2. For $\operatorname{EDS}(\Omega, \star, \star), \varphi_{\leftarrow}$ and $\varphi_{\rightarrow}$ are surjective.

3. $(\Omega, \star)$ is a group.

If this holds, for any $\alpha, \beta \in \Omega$ :

$$
\begin{aligned}
\alpha \curvearrowleft \beta & =\alpha \star \beta^{-1}, & \alpha \frown \beta & =\alpha^{-1} \star \beta, \\
\alpha \triangleleft \beta & =\beta, & \alpha \bullet \beta & =\alpha .
\end{aligned}
$$

Proof. Obviously, $1 . \Longrightarrow 2$.

2. $\Longrightarrow 3$. For any $\alpha, \beta \in \Omega, \varphi_{\leftarrow}(\alpha, \beta)=(\alpha \star \beta, \beta)$ and $\varphi_{\rightarrow}(\alpha, \beta)=(\alpha \star \beta, \alpha)$. Hence, for any $\beta, \gamma \in \Omega$, there exist $\alpha, \alpha^{\prime} \in \Omega$, such that $\alpha \star \beta=\beta \star \alpha^{\prime}=\gamma$.

Let us fix $\beta_{0} \in \Omega$, and let us consider elements $e$ and $e^{\prime}$ such that $e \star \beta_{0}=\beta_{0} \star e^{\prime}=\beta_{0}$. Let $\gamma \in \Omega$; there exists $\alpha \in \Omega$, such that $\alpha \star \beta_{0}=\gamma$. Hence,

$$
\gamma \star e^{\prime}=\alpha \star \beta_{0} \star e^{\prime}=\alpha \star \beta_{0}=\gamma
$$

Similarly, $e \star \gamma=\gamma$ for any $\gamma$. In particular $e \star e^{\prime}=e=e^{\prime}$ is a unit of $\Omega$. For any $\beta \in \Omega$, there exist $\beta^{\prime}, \beta^{\prime \prime} \in \Omega$, such that

$$
\beta^{\prime} \star \beta=\beta \star \beta^{\prime \prime}=e .
$$

Moreover, $\beta^{\prime} \star \beta \star \beta^{\prime \prime}=\beta^{\prime} \star e=\beta^{\prime}=e \star \beta^{\prime \prime}=\beta^{\prime \prime}$, so $\beta^{\prime}=\beta^{\prime \prime}$ is an inverse of $\beta$ in $\Omega$ : $\Omega$ is a group.

3. $\Longrightarrow 1$. The inverse bijections of $\varphi_{\leftarrow}$ and $\varphi_{\rightarrow}$ are given by

$$
\varphi_{\leftarrow}^{-1}(\alpha, \beta)=\left(\alpha \star \beta^{-1}, \beta\right), \quad \varphi_{\rightarrow}^{-1}(\alpha, \beta)=\left(\beta, \beta^{-1} \star \alpha\right) .
$$

So $\Omega$ is nondegenerate.

Proposition 8. Let $\Omega=(\Omega, \star, \star, \triangleleft, \triangleright)$ be an associative semigroup. We assume that, either $\Omega$ is finite, or either $(\Omega, \star)$ is cancellative: for any $\alpha, \beta, \gamma \in \Omega$,

$$
(\alpha \star \beta=\alpha \star \gamma) \Longrightarrow(\beta=\gamma), \quad(\alpha \star \gamma=\beta \star \gamma) \Longrightarrow(\alpha=\beta) .
$$

Then $\Omega$ is nondegenerate if, and only if, the two following conditions hold:

1. $(\Omega, \star)$ is a group.

2. $\Omega=\operatorname{EDS}(\Omega, \star, \star)$. 
Proof. $1 . \Longrightarrow 2$. Let us assume that $\Omega$ is nondegenerate. We consider the map:

$$
\psi:\left\{\begin{aligned}
\Omega^{3} & \longrightarrow \Omega^{3} \\
(\alpha, \beta, \gamma) & \longrightarrow(\alpha \triangleright \beta, \beta \triangleright \gamma, \alpha \star \beta \star \gamma) .
\end{aligned}\right.
$$

Let us prove that $\psi$ is injective. We denote by $\psi_{\rightarrow}=\left(\psi_{\rightarrow}^{1}, \psi_{\rightarrow}^{2}\right)$ the inverse of the bijection $\varphi \rightarrow$. We put:

$$
\psi^{\prime}:\left\{\begin{aligned}
\Omega^{3} & \longrightarrow \Omega^{3} \\
(\alpha, \beta, \gamma) & \longrightarrow\left(\psi_{\rightarrow}^{1}\left(\psi_{\rightarrow}^{1}(\gamma, \alpha \star \beta), \alpha\right), \psi_{\rightarrow}^{2}\left(\psi_{\rightarrow}^{1}(\gamma, \alpha \star \beta), \alpha\right), \psi_{\rightarrow}^{1}(\gamma, \alpha \star \beta)\right) .
\end{aligned}\right.
$$

Let $\alpha, \beta, \gamma \in \Omega$. We put $\psi^{\prime} \circ \psi(\alpha, \beta, \gamma)=\left(\alpha^{\prime}, \beta^{\prime}, \gamma^{\prime}\right)$ with:

$$
\begin{aligned}
& \alpha^{\prime}=\psi_{\rightarrow}^{1}\left(\psi_{\rightarrow}^{1}(\alpha \star \beta \star \gamma,(\alpha \triangleright \beta) \star(\beta \triangleright \gamma)), \alpha \triangleright \beta\right), \\
& \beta^{\prime}=\psi_{\rightarrow}^{2}\left(\psi_{\rightarrow}^{1}(\alpha \star \beta \star \gamma,(\alpha \triangleright \beta) \star(\beta \triangleright \gamma)), \alpha \triangleright \beta\right), \\
& \gamma^{\prime}=\psi_{\rightarrow}^{2}(\alpha \star \beta \star \gamma,(\alpha \triangleright \beta) \star(\beta \triangleright \gamma)) .
\end{aligned}
$$

By 19$)$ :

$$
\varphi \rightarrow(\alpha \star \beta, \gamma)=(\alpha \star \beta \star \gamma,(\alpha \triangleright \beta) \star(\beta \triangleright \gamma)),
$$

Therefore:

$$
\alpha^{\prime}=\psi_{\rightarrow}^{1}(\alpha \star \beta, \alpha \triangleright \beta)=\alpha, \quad \beta^{\prime}=\psi_{\rightarrow}^{2}(\alpha \star \beta, \alpha \triangleright \beta)=\beta, \quad \gamma^{\prime}=\gamma .
$$

So $\psi$ is injective. If $\Omega$ is finite, $\psi$ is bijective. If $\Omega$ is cancellative, let us put $\psi^{\prime}(\alpha, \beta, \gamma)=$ $\left(\alpha^{\prime}, \beta^{\prime}, \gamma^{\prime}\right)$. By definition of $\psi$, the first component of $\psi\left(\alpha^{\prime}, \beta^{\prime}, \gamma^{\prime}\right)$ is $\alpha$ and the third one is $\gamma$. Let us denote its second component by $\beta^{\prime \prime}=\beta^{\prime} \triangleright \gamma^{\prime}$. By definition of $\psi^{\prime}$ :

$$
\alpha^{\prime} \star \beta^{\prime} \star \gamma^{\prime}=\gamma, \quad\left(\alpha^{\prime} \star \beta^{\prime}\right) \triangleright \gamma^{\prime}=\alpha \star \beta, \quad \alpha^{\prime} \triangleright \beta^{\prime}=\alpha .
$$

Moreover, by (19):

$$
\alpha \star \beta=\left(\alpha^{\prime} \star \beta^{\prime}\right) \triangleright \gamma^{\prime}=\left(\alpha^{\prime} \triangleright \beta^{\prime}\right) \star\left(\beta^{\prime} \triangleright \gamma^{\prime}\right)=\alpha \star\left(\beta^{\prime} \triangleright \gamma^{\prime}\right)=\alpha \star \beta^{\prime \prime} .
$$

As $\Omega$ is cancellative, $\beta^{\prime \prime}=\beta$, so $\psi \circ \psi^{\prime}=I d_{\Omega}$ and $\psi$ is surjective. (21):

Consequently, if $\alpha^{\prime}, \beta^{\prime} \in \Omega$, there exist $\alpha, \beta, \gamma \in \Omega$, such that $\alpha^{\prime}=\alpha \triangleleft \beta$ and $\beta^{\prime}=\beta \triangleleft \gamma$. By

$$
\alpha^{\prime} \triangleright \beta^{\prime}=\alpha^{\prime} .
$$

We prove similarly that $\alpha^{\prime} \triangleleft \beta^{\prime}=\beta^{\prime}$, using (17). By Lemma 7 , $(\Omega, \star)$ is a group.

2. $\Longrightarrow 1$. The inverse implication comes from Lemma 7 .

Proposition 9. Let $(H, \star)$ be a group, $K$ be a nonempty set and $\theta: K \longrightarrow H$ be a map. We define four products on $H \times K$ in the following way:

$$
\begin{aligned}
& \forall\left(\alpha, \alpha^{\prime}\right),\left(\beta, \beta^{\prime}\right) \in H \times K, \quad\left(\alpha, \alpha^{\prime}\right) \leftarrow\left(\beta, \beta^{\prime}\right)=\left(\alpha, \alpha^{\prime}\right), \\
&\left(\alpha, \alpha^{\prime}\right) \rightarrow\left(\beta, \beta^{\prime}\right)=\left(\beta, \beta^{\prime}\right), \\
&\left(\alpha, \alpha^{\prime}\right) \triangleleft\left(\beta, \beta^{\prime}\right)=\left(\alpha^{-1} \star \beta, \beta^{\prime}\right), \\
&\left(\alpha, \alpha^{\prime}\right) \triangleright\left(\beta, \beta^{\prime}\right)=\left(\theta\left(\beta^{\prime}\right) \star \beta^{-1} \star \alpha, \alpha^{\prime}\right) .
\end{aligned}
$$

This defines a nondegenerate EDS denoted by $\operatorname{EDS}^{*}(H, \star, K, \theta)$. It is commutative if, and only if, for any $\alpha^{\prime} \in K, \theta\left(\alpha^{\prime}\right)$ is the unit of $H$. For any $\left(\alpha, \alpha^{\prime}\right),\left(\beta, \beta^{\prime}\right) \in H \times K$ :

$$
\begin{aligned}
& \left(\alpha, \alpha^{\prime}\right) \curvearrowleft\left(\beta, \beta^{\prime}\right)=\left(\alpha, \alpha^{\prime}\right), \\
& \left(\alpha, \alpha^{\prime}\right) \triangleleft\left(\beta, \beta^{\prime}\right)=\left(\alpha \star \beta, \beta^{\prime}\right), \\
& \left(\alpha, \alpha^{\prime}\right) \triangleleft\left(\beta, \beta^{\prime}\right)=\left(\beta, \beta^{\prime}\right), \\
& \left(\alpha, \alpha^{\prime}\right) \bullet\left(\beta, \beta^{\prime}\right)=\left(\beta \star \theta\left(\beta^{\prime}\right) \star \alpha, \alpha^{\prime}\right) \text {. }
\end{aligned}
$$


Proof. Note that $(H \times K, \leftarrow, \rightarrow)=\mathbf{D S}(H \times K)$. Direct computations prove that (22)-(25) are satisfied. Moreover, $\varphi \leftarrow$ and $\varphi \rightarrow$ are bijections, which inverses are given by:

$$
\left.\varphi_{\leftarrow}^{-1}\left(\left(\alpha, \alpha^{\prime}\right),\left(\beta, \beta^{\prime}\right)\right)=\left(\left(\alpha, \alpha^{\prime}\right),\left(\alpha \star \beta, \beta^{\prime}\right)\right), \quad \varphi_{\rightarrow}^{-1}\left(\left(\alpha, \alpha^{\prime}\right),\left(\beta, \beta^{\prime}\right)\right)=\left(\alpha \star \theta\left(\alpha^{\prime}\right) \star \beta, \beta^{\prime}\right),\left(\alpha, \alpha^{\prime}\right)\right) .
$$

So this EDS is nondegenerate.

Example 3. 1. If $K$ is reduced to a single element, let us denote by $\omega$ the image of this element by $\theta$. As a set, $\operatorname{EDS}^{*}(H, \star, \Omega, \theta)$ is identified with $H$, given the products:

$$
\begin{aligned}
\alpha \leftarrow \beta & =\alpha, & \alpha \rightarrow \beta & =\beta, \\
\alpha \triangleleft \beta & =\alpha^{-1} \star \beta, & \alpha \triangleright \beta & =\omega \star \beta^{-1} \star \alpha .
\end{aligned}
$$

This diassociative semigroup will be denoted by $\operatorname{EDS}^{*}(H, \star, \omega)$. It is commutative if, and only if, $\omega$ is the unit of $H$. In this case, we shall simply denote it by $\operatorname{EDS}^{*}(H, \star)$.

2. If $H$ is a null group, we identify $H \times K$ and $K$. We obtain $\operatorname{EDS}(K)$.

Proposition 10. Let $(\Omega, \leftarrow, \rightarrow, \triangleleft, \triangleright)$ be a finite nondegenerate EDS, such that $(\Omega, \leftarrow, \rightarrow)=$ $\operatorname{DS}(\Omega)$. There exist a group $(H, \star)$, a nonempty set $K$ and a map $\theta: K \longrightarrow H$ such that $\Omega$ is isomorphic to $\mathbf{E D S}^{*}(H, \star, K, \theta)$.

Proof. First step. For any $\alpha, \beta \in \Omega, \varphi_{\leftarrow}(\alpha, \beta)=(\alpha, \alpha \triangleleft \beta)$ and $\varphi_{\rightarrow}(\alpha, \beta)=(\beta, \alpha \triangleright \beta)$. With the notations of Proposition 6, for any $\alpha, \beta \in \Omega$ :

$$
\alpha \curvearrowleft \beta=\alpha, \quad \alpha \frown \beta=\beta .
$$

Moreover, for any $\alpha, \beta, \gamma \in \Omega$ :

$$
\alpha \triangleleft \beta=\gamma \Longleftrightarrow \beta=\alpha \triangleleft \gamma, \quad \alpha \triangleright \beta=\gamma \Longleftrightarrow \alpha=\gamma \bullet \beta .
$$

The relations of Proposition 6 simplify: for any $\alpha, \beta, \gamma \in \Omega$,

$$
\begin{aligned}
& \alpha \triangleleft(\beta \triangleleft \gamma)=(\alpha \triangleleft \beta) \triangleleft \gamma, \\
& \alpha \bullet(\beta \bullet \gamma)=(\alpha \bullet \beta) \bullet \gamma, \\
& (\alpha \triangleleft \beta) \bullet \gamma=(\alpha \bullet \gamma) \triangleleft \beta \text {, } \\
& \alpha \hookrightarrow(\beta \bullet \gamma)=\beta \bullet(\alpha \hookrightarrow \gamma) \text {. }
\end{aligned}
$$

Second step. Let us study the semigroup $(\Omega, \triangleleft)$. For any $\alpha \in \Omega$, we consider the map

$$
f_{\alpha}:\left\{\begin{array}{lll}
\Omega & \longrightarrow & \Omega \\
\beta & \longrightarrow & \alpha \triangleleft \beta
\end{array}\right.
$$

This is an element of the symmetric group $\mathfrak{S}(\Omega)$. By $(39)$, for any $\alpha, \beta \in \Omega$ :

$$
f_{\alpha} \circ f_{\beta}=f_{\alpha \triangleleft \beta} .
$$

Hence, if $H^{\prime}=\left\{f_{\alpha}, \alpha \in \Omega\right\}, H^{\prime}$ is a sub-semigroup of $\mathfrak{S}(\Omega)$. As $\Omega$ is finite, this is a subgroup of $\mathfrak{S}(\Omega)$. Consequently, the following set is nonempty:

$$
K=\left\{\alpha \in \Omega, f_{\alpha}=I d_{\Omega}\right\}=\{\alpha \in \Omega, \forall \beta \in \Omega, \alpha \triangleleft \beta=\beta\} .
$$

Let us choose $e \in K$. We consider the map

$$
\psi:\left\{\begin{array}{rll}
H^{\prime} & \longrightarrow & \Omega \\
f & \longrightarrow & f(e) .
\end{array}\right.
$$

For any $\alpha, \beta \in \Omega$, as $e \hookrightarrow \beta=\beta$ :

$$
\psi\left(f_{\alpha} \circ f_{\beta}\right)=\alpha \triangleleft \beta \hookrightarrow e=\alpha \triangleleft e \triangleleft \beta \hookrightarrow e=f_{\alpha}(e) \triangleleft f_{\beta}(e) .
$$


So $\psi$ is a semigroup morphism. Let us assume that $\psi\left(f_{\alpha}\right)=\psi\left(f_{\beta}\right)$. For any $\gamma \in \Omega$ :

$$
f_{\alpha}(\gamma)=\alpha \triangleleft \gamma=\alpha \triangleleft e \triangleleft \gamma=\psi\left(f_{\alpha}\right) \triangleleft \gamma=\psi\left(f_{\beta}\right) \triangleleft \gamma=f_{\beta}(\gamma)
$$

so $f_{\alpha}=f_{\beta}: \psi$ is injective. Let us denote by $H$ its image; then $H$ is a sub-semigroup of $(\Omega, \triangleleft)$ and is a group, of unit $e$. For any $\beta=f_{\alpha}(e) \in \Omega$, for any $\gamma \in \Omega$ :

$$
f_{\beta}(\gamma)=f_{\alpha}(e) \triangleleft \gamma=\alpha \triangleleft e \triangleleft \gamma=\alpha \triangleleft \gamma=f_{\alpha}(\gamma),
$$

so $f_{\beta}=f_{\alpha}$. Hence, the inverse of $\psi$ is:

$$
\psi^{-1}:\left\{\begin{array}{rll}
H & \longrightarrow & H^{\prime} \\
\beta & \longrightarrow & f_{\beta} .
\end{array}\right.
$$

We denote by $\star$ the product of $H$ : for any $\alpha, \beta \in H, \alpha \star \beta=\alpha \triangleleft \beta$. We define a product on $H \times K$ by:

$$
\left(\alpha, \alpha^{\prime}\right) \triangleleft\left(\beta, \beta^{\prime}\right)=\left(\alpha \star \beta, \beta^{\prime}\right)
$$

Let us consider the map

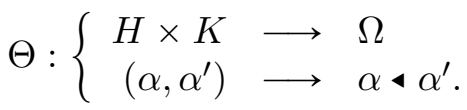

For any $\left(\alpha, \alpha^{\prime}\right),\left(\beta, \beta^{\prime}\right) \in H \times K$, as $\alpha^{\prime} \in K$ :

$$
\Theta\left(\left(\alpha, \alpha^{\prime}\right) \bullet\left(\beta, \beta^{\prime}\right)\right)=\Theta\left(\alpha \star \beta, \beta^{\prime}\right)=\alpha \bullet \beta \bullet \beta^{\prime}=\alpha \bullet \alpha^{\prime} \bullet \beta \bullet \beta^{\prime}=\Theta\left(\alpha, \alpha^{\prime}\right) \triangleleft \Theta\left(\beta, \beta^{\prime}\right) .
$$

So $\Theta$ is a semigroup morphism.

Let $\left(\alpha, \alpha^{\prime}\right),\left(\beta, \beta^{\prime}\right) \in H \times K$, such that $\Theta\left(\alpha, \alpha^{\prime}\right)=\Theta\left(\beta, \beta^{\prime}\right)$. Hence, $\alpha \triangleleft \alpha^{\prime}=\beta \bullet \beta^{\prime}$. Therefore, as $\alpha^{\prime}, \beta^{\prime} \in K$ :

$$
f_{\alpha}=f_{\alpha} \circ f_{\alpha^{\prime}}=f_{\alpha \triangleleft \alpha^{\prime}}=f_{\beta \triangleleft \beta^{\prime}}=f_{\beta} \circ f_{\beta^{\prime}}=f_{\beta} .
$$

As $\psi^{-1}$ is injective, $\alpha=\beta$. Consequently:

$$
\alpha^{\prime}=\alpha \triangleleft\left(\alpha \triangleleft \alpha^{\prime}\right)=\beta \triangleleft\left(\beta \triangleleft \beta^{\prime}\right)=\beta^{\prime} .
$$

So $\Theta$ is injective.

Let $\gamma \in \Omega$. There exists a unique $\alpha \in H$, such that $f_{\alpha}=f_{\gamma}$. Let $\alpha^{-1}$ be the inverse of $\alpha$ in the group $H$ and $\alpha^{\prime}=\alpha^{-1} \triangleleft \gamma$. Then:

$$
\alpha \triangleleft \alpha=\alpha \triangleleft \alpha^{-1} \triangleleft \gamma=f_{\alpha \triangleleft \alpha^{-1}}(\gamma)=I d_{\Omega}(\gamma)=\gamma .
$$

Moreover, for any $\beta \in \Omega$ :

$$
f_{\alpha^{\prime}}=f_{\alpha^{-1}} \circ f_{\gamma}=f_{\alpha}^{-1} \circ f_{\gamma}=f_{\gamma}^{-1} \circ f_{\gamma}=I d_{\Omega},
$$

so $\alpha^{\prime} \in K$ and $\gamma=\Theta\left(\alpha, \alpha^{\prime}\right)$ : $\Theta$ is surjective.

From now, we assume, up to an isomorphism, that $(\Omega, \triangleleft)=(H \times K, \mathbf{\triangleleft})$. By definition of $\mathbf{\bullet}$, for any $\left(\alpha, \alpha^{\prime}\right),\left(\beta, \beta^{\prime}\right) \in H \times K$,

$$
\left(\alpha, \alpha^{\prime}\right) \triangleleft\left(\beta, \beta^{\prime}\right)=\left(\alpha^{-1} \star \beta, \beta^{\prime}\right) .
$$

Last step. Let us now study the product $\bullet$. Recall that $e$ is the unit of $H$ and let us choose $\alpha \in K$. We put, for any $\left(\gamma, \gamma^{\prime}\right) \in H \times K$ :

$$
(e, a) \bullet\left(\gamma, \gamma^{\prime}\right)=\left(\iota\left(\gamma, \gamma^{\prime}\right), \iota^{\prime}\left(\gamma, \gamma^{\prime}\right)\right) .
$$

By 40, , for any $\left(\alpha, \alpha^{\prime}\right),\left(\beta, \beta^{\prime}\right) \in H \times K$ :

$$
\left(\beta, \beta^{\prime}\right) \bullet\left(\gamma, \gamma^{\prime}\right)=\left((e, \alpha) \bullet\left(\beta, \beta^{\prime}\right)\right) \bullet\left(\gamma, \gamma^{\prime}\right)=\left((e, \alpha) \bullet\left(\gamma, \gamma^{\prime}\right)\right) \bullet\left(\beta, \beta^{\prime}\right)=\left(\iota\left(\gamma, \gamma^{\prime}\right) \star \beta, \beta^{\prime}\right) .
$$


Still by 40 :

$$
\begin{aligned}
\left(\alpha, \alpha^{\prime}\right) \triangleleft\left((\beta, \beta)^{\prime} \bullet\left(\gamma, \gamma^{\prime}\right)\right) & =\left(\alpha \star \iota\left(\gamma, \gamma^{\prime}\right) \star \beta, \beta^{\prime}\right) \\
& =\left(\beta, \beta^{\prime}\right) \bullet\left(\left(\alpha, \alpha^{\prime}\right) \triangleleft\left(\gamma, \gamma^{\prime}\right)\right. \\
& =\left(\iota\left(\alpha \star \gamma, \gamma^{\prime}\right) \star \beta, \beta^{\prime}\right) .
\end{aligned}
$$

Hence, $\iota\left(\alpha \star \gamma, \gamma^{\prime}\right)=\alpha \star \iota\left(\gamma, \gamma^{\prime}\right)$. We put $\theta\left(\alpha^{\prime}\right)=\iota\left(e, \alpha^{\prime}\right)^{-1}$. For any $\alpha, \alpha^{\prime} \in K$, taking $\gamma=e$ :

$$
\iota\left(\alpha, \alpha^{\prime}\right)=\alpha \star \iota\left(e, \alpha^{\prime}\right)=\alpha \star \theta\left(\alpha^{\prime}\right)^{-1} .
$$

Finally, for any $\left(\alpha, \alpha^{\prime}\right),\left(\beta, \beta^{\prime}\right) \in H \times K$ :

$$
\left(\alpha, \alpha^{\prime}\right) \bullet\left(\beta, \beta^{\prime}\right)=\left(\beta \star \theta\left(\beta^{\prime}\right)^{-1} \star \alpha, \alpha^{\prime}\right) .
$$

By definition of $\bullet,\left(\alpha, \alpha^{\prime}\right) \triangleright\left(\beta, \beta^{\prime}\right)=\left(\theta\left(\beta^{\prime}\right) \star \beta^{-1} \star \alpha, \alpha^{\prime}\right)$. So $\Omega=\mathbf{E D S}^{*}(H, \star, K, \theta)$.

\subsection{Extended diassociative semigroups of cardinality two}

Let $\Omega=\{a, b\}$ be a set of cardinality two. There are 16 maps from $\Omega^{2}$ to $\Omega$. Testing all possibilities with a computer, we find 13 structures of diassociative semigroups on $\Omega$, which restrict to 8 up to isomorphism, and 45 structures of $\operatorname{EDS}$ on $\Omega$, which restrict to 24 up to isomorphism. In order to describe them, we shall use the maps $\phi_{a}, \phi_{b}: \Omega \longrightarrow \Omega$, such that for any $\alpha \in \Omega$ :

$$
\phi_{a}(\alpha)=a, \quad \phi_{b}(\alpha)=b .
$$

We shall meet six possible products for $\triangleleft$ and $\triangleright$, denoted by:

\begin{tabular}{|c|c|c|}
\hline$m_{a}$ & $a$ & $b$ \\
\hline$a$ & $a$ & $a$ \\
\hline$b$ & $a$ & $a$ \\
\hline
\end{tabular}

\begin{tabular}{|c|c|c|}
\hline$m_{b}$ & $a$ & $b$ \\
\hline$a$ & $b$ & $b$ \\
\hline$b$ & $b$ & $b$ \\
\hline
\end{tabular}

\begin{tabular}{|c|c|c|}
\hline$\triangleleft_{\text {EDS }}$ & $a$ & $b$ \\
\hline$a$ & $a$ & $b$ \\
\hline$b$ & $a$ & $b$ \\
\hline
\end{tabular}

\begin{tabular}{|c|c|c|}
\hline$\triangleright_{\text {EDS }}$ & $a$ & $b$ \\
\hline$a$ & $a$ & $a$ \\
\hline$b$ & $b$ & $b$ \\
\hline
\end{tabular}

\begin{tabular}{|c|c|c|}
\hline$m_{1}$ & $a$ & $b$ \\
\hline$a$ & $a$ & $b$ \\
\hline$b$ & $b$ & $a$ \\
\hline
\end{tabular}

\begin{tabular}{|c|c|c|}
\hline$m_{2}$ & $a$ & $b$ \\
\hline$a$ & $b$ & $a$ \\
\hline$b$ & $a$ & $b$ \\
\hline
\end{tabular}

A.

\begin{tabular}{|c|c|c|}
\hline$\leftarrow A=\rightarrow_{A}$ & $a$ & $b$ \\
\hline$a$ & $a$ & $a$ \\
\hline$b$ & $a$ & $a$ \\
\hline
\end{tabular}

This is the diassociative semigroup attached to the semigroup such that:

$$
\forall \alpha, \beta \in \Omega, \quad \alpha \star_{A} \beta=a .
$$

A1. $\left(\{a, b\}, \star_{A},{ }^{\star}{ }_{A}, m_{a}, m_{a}\right)$. This is $\operatorname{EDS}\left(\{a, b\}, \star_{A},{ }^{\star}{ }_{A}, \phi_{a}, \phi_{a}\right)$.

It is commutative.

A2. $\left(\{a, b\},{ }^{\star} A, \star_{A}, \triangleleft_{\mathbf{E D S}}, \triangleright_{\mathbf{E D S}}\right)$. This is $\operatorname{EDS}\left(\{a, b\},{ }^{\star} A,{ }^{\star} A\right)$.

It is commutative.

B.

\begin{tabular}{|c|c|c|}
\hline$\leftarrow_{B}$ & $a$ & $b$ \\
\hline$a$ & $a$ & $a$ \\
\hline$b$ & $a$ & $a$ \\
\hline
\end{tabular}

\begin{tabular}{|c|c|c|}
\hline$\rightarrow_{B}$ & $a$ & $b$ \\
\hline$a$ & $a$ & $b$ \\
\hline$b$ & $a$ & $b$ \\
\hline
\end{tabular}


B1. $\left(\{a, b\}, \leftarrow_{B}, \rightarrow_{B}, m_{a}, m_{a}\right)$. This is $\operatorname{EDS}\left(\{a, b\}, \leftarrow_{B}, \rightarrow_{B}, \phi_{a}, \phi_{a}\right)$. It is the opposite of D1.

B2. $\left(\{a, b\}, \leftarrow_{B}, \rightarrow_{B}, \triangleleft_{\text {EDS }}, \triangleright_{\text {EDS }}\right)$. This is $\operatorname{EDS}\left(\{a, b\}, \leftarrow_{B}, \rightarrow_{B}\right)$. It is the opposite of D2.

C.

\begin{tabular}{|c|c|c|}
\hline$\leftarrow_{C}=\rightarrow_{C}$ & $a$ & $b$ \\
\hline$a$ & $a$ & $a$ \\
\hline$b$ & $a$ & $b$ \\
\hline
\end{tabular}

This is the diassociative semigroup $(\mathbb{Z} / 2 \mathbb{Z}, \times, \times)$, with $a=\overline{0}$ and $b=\overline{1}$.

C1. $\left(\{a, b\}, \leftarrow_{C}, \rightarrow_{C}, m_{a}, m_{a}\right)$. This is $\operatorname{EDS}\left(\mathbb{Z} / 2 \mathbb{Z}, \times, \times, \phi_{a}, \phi_{a}\right)$. It is commutative.

C2. $\left(\{a, b\}, \leftarrow_{C}, \rightarrow_{C}, m_{a}, m_{b}\right)$. This is $\operatorname{EDS}\left(\mathbb{Z} / 2 \mathbb{Z}, \times, \times, \phi_{a}, \phi_{b}\right)$. It is the opposite of $\mathrm{C} 4$.

C3. $\left(\{a, b\}, \leftarrow_{C}, \rightarrow_{C}, \triangleleft_{\mathbf{E D S}}, \triangleright_{\mathbf{E D S}}\right)$. This is $\operatorname{EDS}(\mathbb{Z} / 2 \mathbb{Z}, \times, \times)$. It is commutative.

C4. $\left(\{a, b\}, \leftarrow_{C}, \rightarrow_{C}, m_{b}, m_{a}\right)$. This is $\operatorname{EDS}\left(\mathbb{Z} / 2 \mathbb{Z}, \times, \times, \phi_{b}, \phi_{a}\right)$. It is the opposite of $\mathrm{C} 2$.

C5. $\left(\{a, b\}, \leftarrow_{C}, \rightarrow_{C}, m_{b}, m_{b}\right)$. This is $\operatorname{EDS}\left(\mathbb{Z} / 2 \mathbb{Z}, \times, \times, \phi_{b}, \phi_{b}\right)$. It is commutative.

D.

\begin{tabular}{|c|c|c|}
\hline$\leftarrow D$ & $a$ & $b$ \\
\hline$a$ & $a$ & $a$ \\
\hline$b$ & $b$ & $b$ \\
\hline
\end{tabular}

\begin{tabular}{|c|c|c|}
\hline$\rightarrow D$ & $a$ & $b$ \\
\hline$a$ & $a$ & $a$ \\
\hline$b$ & $a$ & $a$ \\
\hline
\end{tabular}

D1. $\left(\{a, b\}, \leftarrow_{D}, \rightarrow_{D}, m_{a}, m_{a}\right)$. This is $\operatorname{EDS}\left(\{a, b\}, \leftarrow_{D}, \rightarrow_{D}, \phi_{a}, \phi_{a}\right)$. It is the opposite of B1.

D2. $\left(\{a, b\}, \leftarrow_{D}, \rightarrow_{D}, \triangleleft_{\text {EDS }}, \triangleright_{\text {EDS }}\right)$. This is $\operatorname{EDS}\left(\{a, b\}, \leftarrow_{D}, \rightarrow_{D}\right)$. It is the opposite of B2.

E.

\begin{tabular}{|c|c|c|}
\hline${ }_{E}=\rightarrow{ }_{E}$ & $a$ & $b$ \\
\hline$a$ & $a$ & $a$ \\
\hline$b$ & $b$ & $b$ \\
\hline
\end{tabular}

This is the diassociative semigroup attached to the semigroup such that:

$$
\forall \alpha, \beta \in \Omega, \quad \alpha \star_{E} \beta=\alpha .
$$

E1. $\left(\{a, b\},{ }^{{ }_{E}},{ }^{\star_{E}}, m_{a}, m_{a}\right)$. This is $\operatorname{EDS}\left(\{a, b\},{ }^{\star} E,{ }^{\star}{ }_{E}, \phi_{a}, \phi_{a}\right)$. It is the opposite of G1.

E2. $\left(\{a, b\},{ }^{\star_{E}},{ }^{\star} E, m_{a}, m_{b}\right)$. This is $\operatorname{EDS}\left(\{a, b\}, \star_{E},{ }^{\star} E, \phi_{a}, \phi_{b}\right)$. It is the opposite of G2.

E3. $\left(\{a, b\}, \star_{E}, \star_{E}, \rightarrow_{D}, \triangleleft_{\mathbf{E D S}}, \triangleright_{\mathbf{E D S}}\right)$. This is $\operatorname{EDS}\left(\{a, b\}, \star_{E}, \star_{E}\right)$. It is the opposite of G3. 
F.

\begin{tabular}{|c|c|c|}
\hline$\leftarrow F$ & $a$ & $b$ \\
\hline$a$ & $a$ & $a$ \\
\hline$b$ & $b$ & $b$ \\
\hline
\end{tabular}

\begin{tabular}{|c|c|c|}
\hline$\rightarrow_{F}$ & $a$ & $b$ \\
\hline$a$ & $a$ & $b$ \\
\hline$b$ & $a$ & $b$ \\
\hline
\end{tabular}

This is $\mathbf{D S}(\{a, b\})$.

F1. $\left(\{a, b\}, \leftarrow_{F}, \rightarrow_{F}, m_{a}, m_{a}\right)$. This is $\operatorname{EDS}\left(\{a, b\}, \leftarrow_{F}, \rightarrow_{F}, \phi_{a}, \phi_{a}\right)$. It is commutative.

F2. $\left(\{a, b\}, \leftarrow_{F}, \rightarrow_{F}, m_{a}, m_{b}\right)$. This is $\operatorname{EDS}\left(\{a, b\}, \leftarrow_{F}, \rightarrow_{F}, \phi_{a}, \phi_{b}\right)$. It is commutative.

F3. $\left(\{a, b\}, \leftarrow_{F}, \rightarrow_{F}, \triangleleft_{\mathbf{E D S}}, \triangleright_{\mathbf{E D S}}\right)$. This is $\operatorname{EDS}(\{a, b\})$. It is commutative.

F4. $\left(\{a, b\}, \leftarrow_{F}, \rightarrow_{F}, m_{1}, m_{1}\right)$. This is $\operatorname{EDS}^{*}(\mathbb{Z} / 2 \mathbb{Z},+)$, with $a=\overline{0}$ and $b=\overline{1}$. It is commutative.

F5. $\left(\{a, b\}, \leftarrow_{F}, \rightarrow_{F}, m_{1}, m_{2}\right)$. This is $\operatorname{EDS}^{*}(\mathbb{Z} / 2 \mathbb{Z},+, \overline{1})$, with $a=\overline{0}$ and $b=\overline{1}$. It is not commutative, but is isomorphic to its opposite via the map permuting $a$ and $b$.

G.

\begin{tabular}{|c|c|c|}
\hline$\leftarrow_{G}=\rightarrow_{G}$ & $a$ & $b$ \\
\hline$a$ & $a$ & $b$ \\
\hline$b$ & $a$ & $b$ \\
\hline
\end{tabular}

This is the diassociative semigroup attached to the semigroup such that:

$$
\forall \alpha, \beta \in \Omega, \quad \alpha \star_{G} \beta=\beta .
$$

G1. $\left(\{a, b\}, \star_{G}, \star_{G}, m_{a}, m_{a}\right)$. This is $\operatorname{EDS}\left(\{a, b\}, \star_{G}, \star_{G}, \phi_{a}, \phi_{a}\right)$. It is the opposite of E1.

G2. $\left(\{a, b\}, \star_{G},{ }^{\star} G, m_{a}, m_{b}\right)$. This is $\operatorname{EDS}\left(\{a, b\},{ }^{\star} G,{ }^{\star} G, \phi_{a}, \phi_{b}\right)$. It is the opposite of E1. It is the opposite of E2.

G3. $\left(\{a, b\}, \star_{G}, \star_{G}, \rightarrow_{D}, \triangleleft_{\mathbf{E D S}}, \triangleright_{\mathbf{E D S}}\right)$. This is $\operatorname{EDS}\left(\{a, b\}, \star_{G}, \star_{G}\right)$. It is the opposite of E3.

H.

$$
\begin{array}{|c|c|c|}
\hline \leftarrow H=\rightarrow H & a & b \\
\hline a & a & b \\
\hline b & b & a \\
\hline
\end{array}
$$

This is the diassociative semigroup attached to the group $(\mathbb{Z} / 2 \mathbb{Z},+)$, with $a=\overline{0}$ and $b=\overline{1}$.

H1. $\left(\{a, b\}, \star_{H}, \star_{H}, m_{a}, m_{a}\right)$. This is $\operatorname{EDS}\left(\mathbb{Z} / 2 \mathbb{Z},+,+, \phi_{a}, \phi_{a}\right)$. It is commutative.

H2. $\left(\{a, b\}, \star_{H},{ }^{\star} H, \rightarrow_{D}, \triangleleft_{\mathbf{E D S}}, \triangleright_{\mathbf{E D S}}\right)$. This is $\operatorname{EDS}(\mathbb{Z} / 2 \mathbb{Z},+,+)$. It is commutative.

Only four of these EDS are nondegenerate: F3, F4, F5, H2.

Remark 2. Similar computations can be done for EDS of cardinality 3. Up to isomorphism, there are four nondegenerate EDS of cardinality 3:

$$
\operatorname{EDS}(\{a, b, c\}), \quad \operatorname{EDS}(\mathbb{Z} / 3 \mathbb{Z},+,+), \quad \operatorname{EDS}^{*}(\mathbb{Z} / 3 \mathbb{Z},+), \quad \operatorname{EDS}^{*}(\mathbb{Z} / 3 \mathbb{Z},+, \overline{1}) .
$$




\section{S-dendriform algebras}

\subsection{Definition and example}

Definition 11. Let $\Omega$ be a set with four products $\leftarrow, \rightarrow, \triangleleft, \triangleright$. A $\Omega$-dendriform algebra is a family $\left(A,\left(<_{\alpha}\right)_{\alpha \in \Omega},\left(>_{\alpha}\right)_{\alpha \in \Omega}\right)$ where $A$ is a vector space and $<_{\alpha},>_{\alpha}: A \otimes A \longrightarrow A$, such that for any $x, y, z \in A$, for any $\alpha, \beta \in \Omega$ :

$$
\begin{aligned}
\left(x<_{\alpha} y\right)<_{\beta} z & =x<_{\alpha \leftarrow \beta}\left(y \prec_{\alpha \triangleleft \beta} z\right)+x \prec_{\alpha \rightarrow \beta}\left(y>_{\alpha \triangleright \beta} z\right), \\
x>_{\alpha}\left(<_{\beta} z\right) & =\left(x>_{\alpha} y\right)<_{\beta} z, \\
x>_{\alpha}\left(y>_{\beta} z\right) & =\left(x>_{\alpha \triangleright \beta} y\right)>_{\alpha \rightarrow \beta} z+\left(x \prec_{\alpha \triangleleft \beta} y\right)>_{\alpha \leftarrow \beta} z .
\end{aligned}
$$

Example 4. 1. If $(\Omega, \star)$ is a semigroup, we recover the definition of dendriform family algebra

14 when we consider $\operatorname{EDS}(\Omega, \star, \star)$ :

$$
\alpha \leftarrow \beta=\alpha \rightarrow \beta=\alpha \star \beta, \quad \alpha \triangleleft \beta=\beta, \quad \alpha \triangleright \beta=\alpha .
$$

Note that in this case, $(\Omega, \leftarrow, \rightarrow)$ is an EDS.

2. For any set $\Omega$, considering $\operatorname{EDS}(\Omega)$, we recover the definition of matching dendriform algebras [7].

Remark 3. Let $A$ be a $\Omega$-dendriform algebra. For any $a, b \in A$, for any $\alpha \in \Omega$, we put:

$$
a \prec_{\alpha}^{o p} b=b>_{\alpha} a, \quad a>_{\alpha}^{o p} b=b<_{\alpha} a .
$$

Then $\left(A,\left(<_{\alpha}^{o p}\right)_{\alpha \in \Omega},\left(>_{\alpha}^{o p}\right)_{\alpha \in \Omega}\right)$ is a $\Omega^{o p}$-dendriform algebra, where the products of $\Omega^{o p}$ are defined by:

$$
\begin{aligned}
& \forall \alpha, \beta \in \Omega, \quad \alpha \leftarrow^{o p} \beta=\beta \rightarrow \alpha, \quad \alpha \rightarrow^{o p} \beta=\beta \leftarrow \alpha, \\
& \alpha \triangleleft^{o p} \beta=\beta \triangleright \alpha, \quad \alpha \triangleright^{o p} \beta=\beta \triangleleft \alpha .
\end{aligned}
$$

This gives the notion of commutative $\Omega$-dendriform algebra:

Definition 12. Let $\Omega$ such that, for any $\alpha, \beta \in \Omega$ :

$$
\alpha \leftarrow \beta=\beta \rightarrow \alpha, \quad \alpha \triangleleft \beta=\beta \triangleright \alpha .
$$

Let $A$ be a $\Omega$-dendriform algebra. We shall say that $A$ is commutative if for any $\alpha, \beta \in \Omega$, for any $a, b \in A$ :

$$
a \prec_{\alpha} b=b>_{\alpha} a .
$$

\subsection{Structures on typed binary trees}

Definition 13. Let $\Omega$ be a set.

1. A $\Omega$-typed binary tree is a pair $(T, \tau)$, where $T$ is a plane binary tree and $\tau$ is a map from the set on internal edges of $T$ to $\Omega$. For any internal edge $e$ of $T, \tau(e)$ is called the type of $e$.

2. The set of $\Omega$-typed binary trees is denoted by $\mathcal{T}_{\Omega}$. We denote by $\mathcal{T}_{\Omega}^{+}$the set of $\Omega$-types binary trees different from the trivial tree 1 .

3. For any $n \geqslant 0$, the set of $\Omega$-typed binary trees with $n$ internal vertices (and $n+1$ leaves) is denoted by $\mathcal{T}_{\Omega}(n)$. 
Consequently:

$$
\mathcal{T}_{\Omega}=\bigsqcup_{n \geqslant 0} \mathcal{T}_{\Omega}(n), \quad \mathcal{T}_{\Omega}^{+}=\bigsqcup_{n \geqslant 1} \mathcal{T}_{\Omega}(n)
$$

Example 5. Here are plane binary trees with $n \leqslant 3$ leaves:

I,
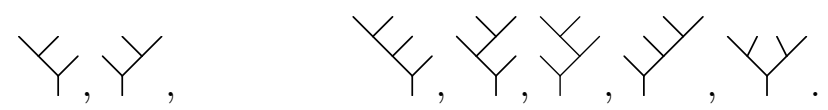

For any $T=(T, \tau) \in \mathcal{T}_{\Omega}$, we shall give indices to internal edges and indicate their types in this way:

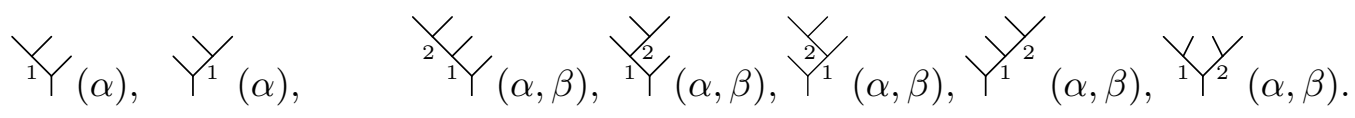

In all cases, the type of the internal edge 1 is $\alpha$ and the type of the internal edge 2 is $\beta$.

Definition 14. Let $T_{1}, T_{2} \in \mathcal{T}_{\Omega}$, and $\alpha, \beta \in \Omega$. We denote by $T_{1} \bigvee_{\alpha, \beta} T_{2}$ the tree $T \in \mathcal{T}_{\Omega}$ obtained by grafting $T_{1}$ on the left and $T_{2}$ and the right on a common root. If $T_{1} \neq \mathrm{I}$, the type of the internal edge between the root of $T$ and the root of $T_{1}$ is $\alpha$. If $T_{2} \neq 1$, the type of internal edge between the root of $T$ and the root of $T_{2}$ is $\beta$.

Example 6. For example, for any $\alpha, \beta, \gamma \in \Omega$ :

$$
\begin{aligned}
& { }^{2} Y(\alpha, \beta)={ }^{Y} Y(\beta) \bigvee_{\alpha, \gamma} \text {, } \\
& { }^{1 / 2}(\alpha, \beta)=Y^{1}(\beta) \bigvee_{\alpha, \gamma} \text { ।, } \\
& Y^{2} /{ }_{1}(\alpha, \beta)=1 \bigvee_{\gamma, \alpha} Y_{(\beta)}, \\
& Y_{1}{ }^{2}(\alpha, \beta)=1 \bigvee_{\gamma, \alpha} Y^{1 / 1}(\beta), \\
& Y Y_{2}(\alpha, \beta)=Y \bigvee_{\alpha, \beta} Y \text {. }
\end{aligned}
$$

Remark 4. Note that any element $T \in \mathcal{T}_{\Omega}(n)$, with $n \geqslant 1$, can be written under the form

$$
T=T_{1} \bigvee_{\alpha, \beta} T_{2}
$$

with $T_{1}, T_{2} \in \mathcal{T}_{\Omega}, \alpha, \beta \in \Omega$. This writing is unique except if $T_{1}=\mid$ or $T_{2}=\mid:$ in this case, one can change arbitrarily $\alpha$ or $\beta$. In order to solve this notational problem, we add an element denoted by $\varnothing$ to $\Omega$ and we shall always assume that if $T_{1}=\mid$, then $\alpha=\varnothing$; if $T_{2}=\mid$, then $\beta=\varnothing$.

Proposition 15. Let $\Omega$ be a set with four products $\leftarrow, \rightarrow, \triangleleft, \triangleright$. We define products $\prec_{\alpha}$ and $>_{\alpha}$ on $\mathbb{K} \mathcal{T}_{\Omega}^{+}$, for $\alpha \in \Omega$, by the following recursive formulas: for any $T, T_{1}, T_{2} \in \mathcal{T}_{\Omega}^{+}$, for any 
$\alpha, \beta, \gamma \in \Omega$,

$$
\begin{aligned}
& Y \prec_{\alpha} T=1 \bigvee_{\varnothing, \alpha} T \\
& \left(T_{1} \bigvee_{\alpha, \varnothing} \text { । }\right)<_{\beta} T=T_{1} \bigvee_{\alpha, \beta} T, \\
& \left(T_{1} \bigvee_{\alpha, \beta} T_{2}\right) \prec_{\gamma} T=T_{1} \bigvee_{\alpha, \beta \leftarrow \gamma}\left(T_{2} \prec_{\beta \triangleleft \gamma} T\right)+T_{1} \bigvee_{\alpha, \beta \rightarrow \gamma}\left(T_{2}>_{\beta \triangleright \gamma} T\right), \\
& T>_{\alpha} Y=T \bigvee_{\alpha, \varnothing} \mid, \\
& T>_{\alpha}\left(\mid \bigvee_{\varnothing, \beta} T_{2}\right)=T \bigvee_{\alpha, \beta} T_{2}, \\
& T>_{\alpha}\left(T_{1} \bigvee_{\beta, \gamma} T_{2}\right)=\left(T>_{\alpha \triangleright \beta} T_{1}\right) \bigvee_{\alpha \rightarrow \beta, \gamma} T_{2}+\left(T \prec_{\alpha \triangleleft \beta} T_{1}\right) \bigvee_{\alpha \leftarrow \beta, \gamma} T_{2} .
\end{aligned}
$$

The following conditions are equivalent:

1. With these products, $\mathbb{K} \mathcal{T}_{\Omega}^{+}$is the free $\Omega$-dendriform freely generated by $Y$.

2. With these products, $\mathbb{K} \mathcal{T}_{\Omega}^{+}$is $\Omega$-dendriform.

3. $(\Omega, \leftarrow, \rightarrow, \triangleleft, \triangleright)$ is an $E D S$.

Proof. We extend the products $<_{\alpha}$ and $>_{\alpha}$ to the space $\mathbb{K} \mathcal{T}_{\Omega}^{+} \otimes \mathbb{K} \mathcal{T}_{\Omega}+\mathbb{K} \mathcal{T}_{\Omega} \otimes \mathbb{K} \mathcal{T}_{\Omega}^{+}$by putting:

$$
\forall x \in \mathbb{K} \mathcal{T}_{\Omega}^{+}, \quad x<_{\alpha}|=|>_{\alpha} x=x, \quad\left|<_{\alpha} x=x>_{\alpha}\right|=0 .
$$

By convention, we consider the added element $\varnothing$ as a unit for the four products of $\Omega$. The definition of the products $<_{\alpha}$ and $>_{\alpha}$ can be rewritten in the following way: for any $T \in \mathcal{T}_{\Omega}^{+}$, for any $T_{1}, T_{2} \in \mathcal{T}_{\Omega}$, for any $\alpha, \beta, \gamma \in \Omega$,

$$
\begin{aligned}
T \prec_{\alpha} \mid & =\mid>_{\alpha} T=T, \\
\mid<_{\alpha} T & =T>_{\alpha} \mid=0, \\
\left(T_{1} \bigvee_{\alpha, \beta} T_{2}\right) \prec_{\gamma} T & =T_{1} \bigvee_{\alpha, \beta \leftarrow \gamma}\left(T_{2} \prec_{\beta \triangleleft \gamma} T\right)+T_{1} \bigvee_{\alpha, \beta \rightarrow \gamma}\left(T_{2}>_{\beta \triangleright \gamma} T\right), \\
T>_{\alpha}\left(T_{1} \bigvee_{\beta, \gamma} T_{2}\right) & =\left(T>_{\alpha \triangleright \beta} T_{1}\right) \bigvee_{\alpha \rightarrow \beta, \gamma} T_{2}+\left(T \prec_{\alpha \triangleleft \beta} T_{1}\right) \bigvee_{\alpha \leftarrow \beta, \gamma} T_{2} .
\end{aligned}
$$

Obviously, $1 . \Longrightarrow 2$. Let us prove that $2 . \Longrightarrow 3$. Let $\alpha, \beta, \gamma \in \Omega$. For $x=y=Y$ and 
$z=Y_{(\gamma)}$

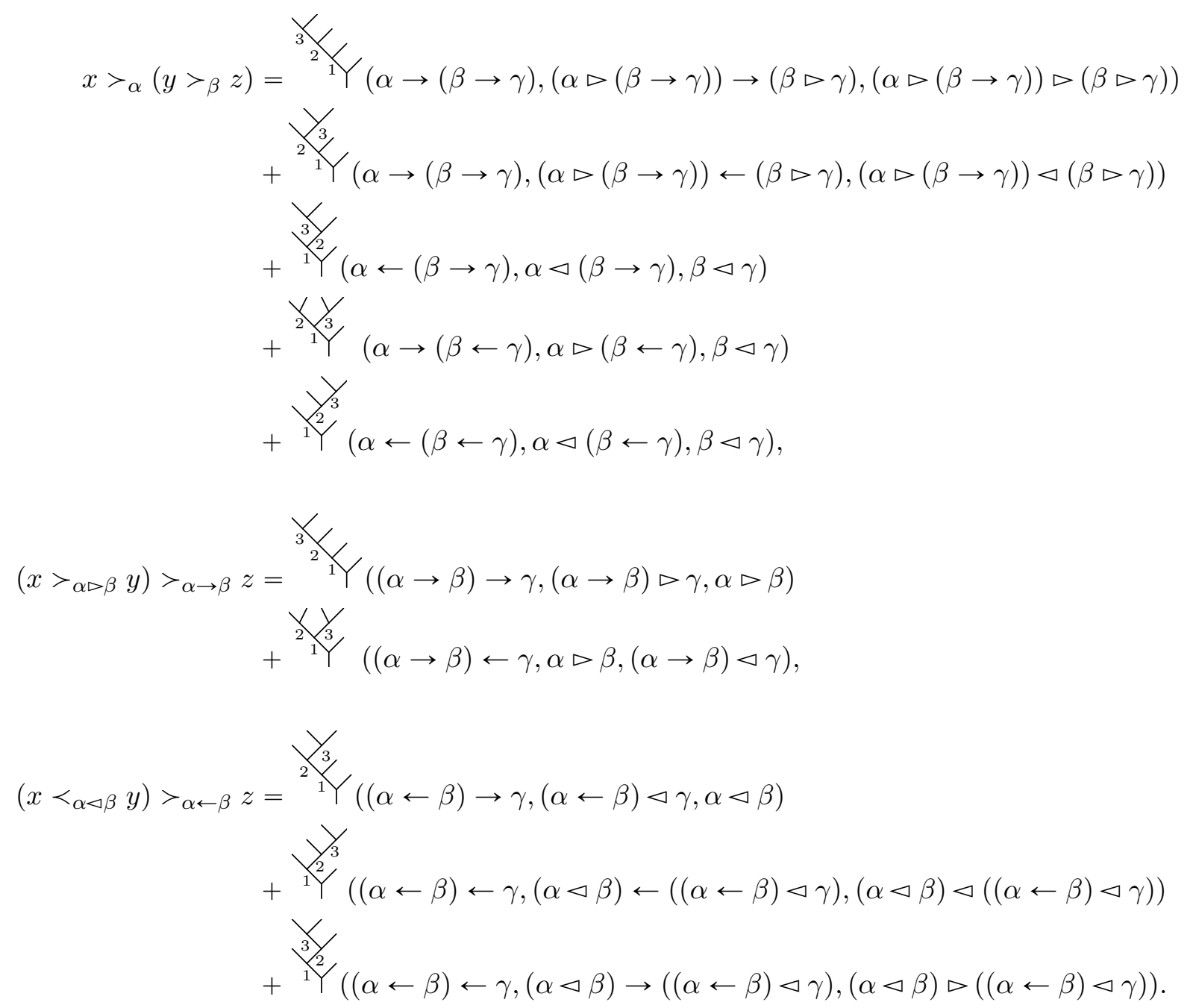

Identifying the decorations of the trees in these expressions, we obtain relations (1)-(13).

3. $\Longrightarrow 1$. Let us first prove that $\mathbb{K} \mathcal{T}_{\Omega}^{+}$is a $\Omega$-dendriform algebra. Let us first prove relations (41)-(43) for $x, y, z \in \mathcal{T}_{\Omega}$ by induction on the total number $N$ of leaves of $x, y$ and $z$. Firstly, observe that (41) is obviously satisfied if $x=1$; 42 is obviously satisfied if $y=\mid$; $(43)$ is obviously satisfied if $z=1$; hence, there is nothing to prove if $N \leqslant 3$. Let us assume the result at all ranks $<N$. Let us first prove 42 for $x, y, z$. We can assume that $y=T_{1} \bigvee_{\gamma, \delta} T_{2}$, where $T_{1}, T_{2} \in \mathcal{T}_{\Omega}$ and $\gamma, \delta \in \Omega \sqcup\{\varnothing\}\left(\gamma=\varnothing\right.$ if $T_{1}=1 ; \delta=\varnothing$ if $\left.\delta=1\right)$. Then:

$$
\begin{aligned}
\left(x>_{\alpha} y\right)<_{\beta} z & =\left(x>_{\alpha \triangleright \gamma} T_{1}\right) \bigvee_{\alpha \rightarrow \gamma, \delta \rightarrow \beta}\left(T_{2}>_{\delta \triangleright \beta} T_{1}\right)+\left(x>_{\alpha \triangleright \gamma} T_{1}\right) \bigvee_{\alpha \rightarrow \gamma, \delta \leftarrow \beta}\left(T_{2}<_{\delta \triangleleft \beta} z\right) \\
& +\left(x \prec_{\alpha \triangleleft \gamma} T_{1}\right) \bigvee_{\alpha \leftarrow \gamma, \delta \rightarrow \beta}\left(T_{2}>_{\delta \triangleright \beta} T_{1}\right)+\left(x \prec_{\alpha \triangleleft \gamma} T_{1}\right) \bigvee_{\alpha \leftarrow \gamma, \delta \leftarrow \beta}\left(T_{2} \prec_{\delta \triangleleft \beta} z\right) \\
& =x>_{\alpha}\left(y<_{\beta} z\right) .
\end{aligned}
$$


Let us now prove 43 for $x, y, z$. We can assume that $z=T_{1} \bigvee_{\gamma, \delta} T_{2}$. Then:

$$
\begin{aligned}
& x>_{\alpha}\left(y>_{\beta} z\right)=\left(x>_{\alpha \triangleright(\beta \rightarrow \gamma)}\left(y>_{\beta \triangleright \gamma} T_{1}\right)\right) \bigvee_{\alpha \rightarrow(\beta \rightarrow \gamma), \delta} T_{2} \\
& +\left(x<_{\alpha \triangleleft(\beta \rightarrow \gamma)}\left(y>_{\beta \triangleright \gamma} T_{1}\right)\right) \bigvee_{\alpha \leftarrow(\beta \rightarrow \gamma), \delta} T_{2} \\
& +\left(x>_{\alpha \triangleright(\beta \leftarrow \gamma)}\left(y<_{\beta \triangleleft \gamma} T_{1}\right)\right) \bigvee_{\alpha \rightarrow(\beta \leftarrow \gamma), \delta} T_{2} \\
& +\left(x \prec_{\alpha \triangleleft(\beta \leftarrow \gamma)}\left(y \prec_{\beta \triangleleft \gamma} T_{1}\right)\right) \bigvee_{\alpha \leftarrow(\beta \leftarrow \gamma), \delta} T_{2}, \\
& \left(x>_{\alpha \triangleright \beta} y\right)>_{\alpha \rightarrow \beta} z=\left(\left(x>_{\alpha \triangleright \beta} y\right)>_{(\alpha \rightarrow \beta) \triangleright \gamma} T_{1}\right) \bigvee_{(\alpha \rightarrow \beta) \rightarrow \gamma, \delta} T_{2} \\
& +\left(\left(x>_{\alpha \triangleright \beta} y\right) \prec_{(\alpha \rightarrow \beta) \triangleleft \gamma} T_{1}\right) \bigvee_{(\alpha \rightarrow \beta) \leftarrow \gamma, \delta} T_{2}, \\
& \left(x \prec_{\alpha \triangleleft \beta} y\right)>_{\alpha \leftarrow \beta} z=\left(\left(x \prec_{\alpha \triangleleft \beta} y\right)>_{(\alpha \leftarrow \beta) \triangleright \gamma} T_{1}\right) \bigvee_{(\alpha \leftarrow \beta) \rightarrow \gamma, \delta} T_{2} \\
& +\left(\left(x \prec_{\alpha \triangleleft \beta} y\right) \prec_{(\alpha \leftarrow \beta) \triangleleft \gamma} T_{1}\right) \bigvee_{(\alpha \leftarrow \beta) \leftarrow \gamma, \delta} T_{2} .
\end{aligned}
$$

Using the induction hypothesis and relations (1)-(13), putting $\alpha^{\prime}=\alpha \triangleright(\beta \rightarrow \gamma)$ and $\beta^{\prime}=\beta \triangleright \gamma$ :

$$
\begin{aligned}
& \left(\left(x>_{\alpha \triangleright \beta} y\right)>_{(\alpha \rightarrow \beta) \triangleright \gamma} T_{1}\right) \bigvee_{(\alpha \rightarrow \beta) \rightarrow \gamma, \delta} T_{2}+\left(\left(x \prec_{\alpha \triangleleft \beta} y\right)>_{(\alpha \leftarrow \beta) \triangleright \gamma} T_{1}\right) \bigvee_{(\alpha \leftarrow \beta) \rightarrow \gamma, \delta} T_{2} \\
& =\left(x>_{\alpha^{\prime}}\left(y>_{\beta^{\prime}} T_{2}\right)\right) \bigvee_{\alpha \rightarrow(\beta \rightarrow \gamma), \delta} T_{2} \\
& \left.=\left(y>_{\beta \triangleright \gamma} T_{1}\right)\right) \bigvee_{\alpha \rightarrow(\beta \rightarrow \gamma), \delta} T_{2} .
\end{aligned}
$$

Similarly:

$$
\left(\left(x>_{\alpha \triangleright \beta} y\right) \prec_{(\alpha \rightarrow \beta) \triangleleft \gamma} T_{1}\right) \bigvee_{(\alpha \rightarrow \beta) \leftarrow \gamma, \delta} T_{2}=\left(x>_{\alpha \triangleright(\beta \leftarrow \gamma)}\left(y<_{\beta \triangleleft \gamma} T_{1}\right)\right) \bigvee_{\alpha \rightarrow(\beta \leftarrow \gamma), \delta} T_{2},
$$

and

$$
\begin{aligned}
& \left(x \prec_{\alpha \triangleleft(\beta \rightarrow \gamma)}\left(y>_{\beta \triangleright \gamma} T_{1}\right)\right) \bigvee_{\alpha \leftarrow(\beta \rightarrow \gamma), \delta} T_{2}+\left(x \prec_{\alpha \triangleleft(\beta \leftarrow \gamma)}\left(y<_{\beta \triangleleft \gamma} T_{1}\right)\right) \bigvee_{\alpha \leftarrow(\beta \leftarrow \gamma), \delta} T_{2} \\
& =\left(\left(x \prec_{\alpha \triangleleft \beta} y\right) \prec_{(\alpha \leftarrow \beta) \triangleleft \gamma} T_{1}\right) \bigvee_{(\alpha \leftarrow \beta) \leftarrow \gamma, \delta} T_{2} .
\end{aligned}
$$

So (43) is satisfied for $x, y, z$. Relation 41 is proved similarly. We obtain that $\mathbb{K} \mathcal{T}_{\Omega}^{+}$is $\Omega$ dendriform.

Let us now prove its freeness. Let $A$ be a $\Omega$-dendriform algebra and let $a \in A$. Let us prove the existence and uniqueness of a $\Omega$-dendriform algebra morphism $\Phi$ from $\mathbb{K} \mathcal{T}_{\Omega}^{+}$to $A$ such that $\Phi(Y)=a$.

We first extend the products of $A$ to $\mathbb{K} \otimes A+A \otimes \mathbb{K}+A \otimes A$ by putting, for any $b \in A$ :

$$
b>_{\alpha} 1=1<_{\alpha} b=0, \quad 1>_{\alpha} b=b<_{\alpha} 1=b .
$$


We then define $\Phi(T)$ for any tree $T \in \mathcal{T}_{\Omega}$ by induction on its number of leaves:

$$
\begin{aligned}
\Phi(\mathrm{I}) & =1, \\
\Phi\left(T_{1} \bigvee_{\alpha, \beta} T_{2}\right) & =\Phi\left(T_{1}\right)>_{\alpha} a<_{\beta} \Phi\left(T_{2}\right) .
\end{aligned}
$$

Let us prove that $\Phi$ is a $\Omega$-dendriform algebra morphism. Let $x, y \in \mathcal{T}_{\Omega}$ and let us prove that

$$
\Phi\left(x>_{\alpha} y\right)=\Phi(x)>_{\alpha} \Phi(y), \quad \Phi\left(x<_{\alpha} y\right)=\Phi(x)<_{\alpha} \Phi(y)
$$

by induction on the total number $N$ of leaves of $x$ and $y$. If $y=1$, then:

$$
\Phi\left(x>_{\alpha} \text { । }\right)=0=\Phi(x)>_{\alpha} 1, \quad \Phi\left(x<_{\alpha} \text { I) }=\Phi(x)=\Phi(x)>_{\alpha} 1 .\right.
$$

The proof is similar if $x=\mathrm{I}$. Let us now assume that $x, y \neq \mathrm{I}$. Let us put $y=T_{1} \bigvee_{\beta, \gamma} T_{2}$. By the induction hypothesis applied to $T_{1}$ :

$$
\begin{aligned}
\Phi\left(x>_{\alpha} y\right) & =\Phi\left(\left(x>_{\alpha \triangleright \beta} T_{1}\right) \bigvee_{\alpha \rightarrow \beta, \gamma} T_{2}\right)+\Phi\left(\left(x<_{\alpha \triangleleft \beta} T_{1}\right) \bigvee_{\alpha \leftarrow \beta, \gamma} T_{2}\right) \\
& =\left(\Phi(x)>_{\alpha \triangleright \beta} \Phi\left(T_{1}\right)\right)>_{\alpha \rightarrow \beta} a<_{\gamma} \Phi\left(T_{2}\right) \\
& +\left(\Phi(x)<_{\alpha \triangleleft \beta} \Phi\left(T_{1}\right)\right)>_{\alpha \leftarrow \beta} a<_{\gamma} \Phi\left(T_{2}\right) \\
& =\left(\Phi(x)>_{\alpha}\left(\Phi\left(T_{1}\right)>_{\beta} a\right)\right)<_{\gamma} \Phi\left(T_{2}\right) \\
& =\Phi(x)>_{\alpha}\left(\left(\Phi\left(T_{1}\right)>_{\beta} a<_{\gamma} \Phi\left(T_{2}\right)\right)\right. \\
& =\Phi(x)>_{\alpha} \Phi(y) .
\end{aligned}
$$

Similarly, $\Phi\left(x \prec_{\alpha} y\right)=\Phi(x) \prec_{\alpha} \Phi(y)$.

Let us now prove the unicity of $\Phi$. Let $\Psi$ be another morphism from $\mathbb{K} \mathcal{T}_{\Omega}^{+}$to $A$ such that $\Psi(Y)=a$. For any tree $T \neq \mathrm{I}$, putting $T=T_{1} \bigvee_{\alpha, \beta} T_{2}$ :

$$
\Psi(T)=\Psi\left(T_{1}>_{\alpha} Y<_{\beta} T_{2}\right)=\Psi\left(T_{1}\right)>_{\alpha} a<_{\beta} \Psi\left(T_{2}\right)
$$

so $\Psi=\Phi$.

Example 7. Let $\alpha, \beta \in \Omega$.

$$
\begin{aligned}
& Y<_{\beta} Y=Y^{1}(\beta) \\
& Y>_{\alpha} Y=Y(\alpha) \text {, } \\
& Y>_{\alpha} Y_{1}(\beta)=\stackrel{2}{1} Y(\alpha \rightarrow \beta, \alpha \triangleright \beta)+1^{1 / 2}(\alpha \leftarrow \beta, \alpha \triangleleft \beta), \\
& Y>_{\alpha} Y^{1 /}(\beta)={ }^{Y} Y_{2}(\alpha, \beta),
\end{aligned}
$$

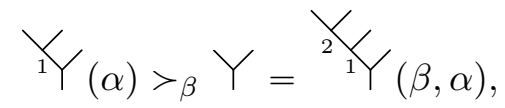

$$
\begin{aligned}
& Y{ }^{1}(\alpha)>_{\beta} Y=1 / 2(\beta, \alpha)
\end{aligned}
$$


Remark 5. 1. An easy induction proves that the $\Omega$-dendriform algebra $\mathbb{K} \mathcal{T}_{\Omega}^{+}$is graded:

$$
\forall \alpha \in \Omega, \forall k, l \geqslant 1, \quad \mathbb{K} \mathcal{T}_{\Omega}(k) \prec_{\alpha} \mathbb{K} \mathcal{T}_{\Omega}(l)+\mathbb{K} \mathcal{T}_{\Omega}(k)>_{\alpha} \mathbb{K} \mathcal{T}_{\Omega}(l) \subseteq \mathbb{K} \mathcal{T}_{\Omega}(k+l)
$$

2. Similar results can be proved for $\Omega$-typed $D$-decorated plane binary trees, that is to say $\Omega$-typed plane binary trees given a map from the set of internal vertices to $D$. We obtain in this way the free $\Omega$-dendriform generated by $D$.

\subsection{Structure on typed words}

Definition 16. Let $\Omega$ be a set and let $V$ be a vector space. The space of $\Omega$-typed words in $V$ is

$$
\mathrm{Sh}_{\Omega}^{+}(V)=\bigoplus_{n \geqslant 1}(\mathbb{K} \Omega)^{\otimes(n-1)} \otimes V^{\otimes n}
$$

Tensors of $\operatorname{Sh}_{\Omega}^{+}(V)$ will be written in the form

$$
\alpha_{2} \ldots \alpha_{n} \otimes v_{1} \ldots v_{n}
$$

where $n \geqslant 1, \alpha_{2}, \ldots, \alpha_{n} \in \Omega$ and $v_{1}, \ldots, v_{n} \in V$. Such a tensor will be called a $\Omega$-typed word in $V$; its length is the integer $n$. We also put $\mathrm{Sh}_{\Omega}(V)=\mathbb{K} \oplus \operatorname{Sh}_{\Omega}^{+}(V)$.

Proposition 17. Let $\Omega$ be a set with four operations $\leftarrow, \rightarrow, \triangleleft, \triangleright$. For any vector space $V$, we give $\mathrm{Sh}_{\Omega}(V)$ products $<_{\alpha},>_{\alpha}$, where $\alpha \in \Omega$, inductively defined in the following way:

$$
\begin{aligned}
& 1 \prec_{\alpha} \alpha_{2} \ldots \alpha_{m} \otimes v_{1} \ldots v_{m}=\alpha_{2} \ldots \alpha_{m} \otimes v_{1} \ldots v_{m}>_{\alpha} 1=0 \\
& \alpha_{2} \ldots \alpha_{m} \otimes v_{1} \ldots v_{m} \prec_{\alpha} 1=1>_{\alpha} \alpha_{2} \ldots \alpha_{n} \otimes v_{1} \ldots v_{m}=\alpha_{2} \ldots \alpha_{m} \otimes v_{1} \ldots v_{m}
\end{aligned}
$$

and

$$
\begin{aligned}
& \alpha_{2} \ldots \alpha_{m} \otimes v_{1} \ldots v_{m} \prec_{\alpha} \beta_{2} \ldots \beta_{n} \otimes w_{1} \ldots w_{n} \\
& =\left(\left(\alpha_{2} \rightarrow \alpha\right) \otimes v_{1}\right) \cdot\left(\alpha_{3} \ldots \alpha_{m} \otimes v_{2} \ldots v_{m} \prec_{\alpha_{2} \triangleleft \alpha} \beta_{2} \ldots \beta_{n} \otimes w_{1} \ldots w_{n}\right) \\
& +\left(\left(\alpha_{2} \leftarrow \alpha\right) \otimes v_{1}\right) \cdot\left(\alpha_{3} \ldots \alpha_{m} \otimes v_{2} \ldots v_{m}>_{\alpha_{2} \triangleright \alpha} \beta_{2} \ldots \beta_{n} \otimes w_{1} \ldots w_{n}\right), \\
& \alpha_{2} \ldots \alpha_{m} \otimes v_{1} \ldots v_{m}>_{\alpha} \beta_{2} \ldots \beta_{n} \otimes w_{1} \ldots w_{n} \\
& =\left(\left(\alpha \rightarrow \beta_{2}\right) \otimes w_{1}\right) \cdot\left(\alpha_{2} \ldots \alpha_{m} \otimes v_{1} \ldots v_{m}<_{\alpha \triangleleft \beta_{2}} \beta_{3} \ldots \beta_{n} \otimes w_{2} \ldots w_{n}\right) \\
& +\left(\left(\alpha \leftarrow \beta_{2}\right) \otimes w_{1}\right) \cdot\left(\alpha_{2} \ldots \alpha_{m} \otimes v_{1} \ldots v_{m}>_{\alpha \triangleright \beta_{2}} \beta_{3} \ldots \beta_{n} \otimes w_{2} \ldots w_{n}\right),
\end{aligned}
$$

where $\cdot$ is the concatenation product:

$$
\cdot\left\{\begin{aligned}
(\mathbb{K} \Omega \otimes V) \otimes \mathrm{Sh}_{\Omega}(V) & \longrightarrow \mathrm{Sh}_{\Omega}^{+}(V) \\
(\alpha \otimes v) \otimes\left(\alpha_{2} \ldots \alpha_{n} \otimes v_{1} \ldots v_{n}\right) & \longrightarrow \alpha \alpha_{2} \ldots \alpha_{n} \otimes v v_{1} \ldots v_{n}
\end{aligned}\right.
$$

The following conditions are equivalent:

1. With these products, $\mathrm{Sh}_{\Omega}^{+}(V)$ is a $\Omega$-dendriform algebra for any vector space $V$.

2. $\Omega$ is an $E D S$.

If this holds and if $\Omega$ is commutative, then $\operatorname{Sh}_{\Omega}^{+}(V)$ is the free commutative $\Omega$-dendriform algebra generated by $V$.

Proof. Note that these products $<_{\alpha},>_{\alpha}$ are defined on

$$
\left(\mathrm{Sh}_{\Omega}(V) \otimes \mathrm{Sh}_{\Omega}(V)\right)^{+}=\mathrm{Sh}_{\Omega}(V) \otimes \operatorname{Sh}_{\Omega}^{+}(V)+\operatorname{Sh}_{\Omega}^{+}(V) \otimes \operatorname{Sh}_{\Omega}(V) .
$$


1. $\Longrightarrow 2$. Let $V$ be a vector space of dimension 4 and $\left(v_{1}, v_{2}, v_{3}, v_{4}\right)$ be a basis of $V$. Let $\alpha, \beta, \gamma \in \Omega$.

$$
\begin{aligned}
& v_{1}>_{\alpha}\left(v_{2}>_{\beta} \gamma \otimes v_{3} v_{4}\right) \\
& =\alpha \rightarrow(\beta \rightarrow \gamma) \cdot(\alpha \triangleright(\beta \rightarrow \gamma)) \rightarrow(\beta \triangleright \gamma) \cdot(\alpha \triangleright(\beta \rightarrow \gamma)) \triangleright(\beta \triangleright \gamma) \otimes v_{3} v_{4} v_{2} v_{1} \\
& +\alpha \rightarrow(\beta \rightarrow \gamma) \cdot(\alpha \triangleright(\beta \rightarrow \gamma)) \leftarrow(\beta \triangleright \gamma) \cdot(\alpha \triangleright(\beta \rightarrow \gamma)) \triangleleft(\beta \triangleright \gamma) \otimes v_{3} v_{4} v_{1} v_{2} \\
& +\alpha \leftarrow(\beta \rightarrow \gamma) \cdot \alpha \triangleleft(\beta \rightarrow \gamma) \cdot \beta \triangleleft \gamma \otimes v_{3} v_{1} v_{4} v_{2} \\
& +\alpha \rightarrow(\beta \leftarrow \gamma) \cdot(\alpha \triangleright(\beta \leftarrow \gamma)) \rightarrow(\beta \triangleleft \gamma) \cdot(\alpha \triangleright(\beta \leftarrow \gamma)) \triangleright(\beta \triangleleft \gamma) \otimes v_{3} v_{2} v_{4} v_{1} \\
& +\alpha \rightarrow(\beta \leftarrow \gamma) \cdot(\alpha \triangleright(\beta \leftarrow \gamma)) \leftarrow(\beta \triangleleft \gamma) \cdot(\alpha \triangleright(\beta \leftarrow \gamma)) \triangleleft(\beta \triangleleft \gamma) \otimes v_{3} v_{2} v_{1} v_{4} \\
& +\alpha \leftarrow(\beta \leftarrow \gamma) \cdot \alpha \triangleleft(\beta \leftarrow \gamma) \cdot \beta \triangleleft \gamma \otimes v_{3} v_{1} v_{2} v_{4}, \\
& \left(v_{1}>_{\alpha \triangleright \beta} v_{2}\right)>_{\alpha \rightarrow \beta} \gamma \otimes v_{3} v_{4} \\
& =(\alpha \rightarrow \beta) \rightarrow \gamma \cdot(\alpha \rightarrow \beta) \triangleright \gamma \cdot \alpha \triangleright \beta \otimes v_{3} v_{4} v_{2} v_{1} \\
& +(\alpha \rightarrow \beta) \leftarrow \gamma \cdot(\alpha \triangleright \beta) \leftarrow((\alpha \rightarrow \beta) \triangleleft \gamma) \cdot(\alpha \triangleright \beta) \triangleleft((\alpha \rightarrow \beta) \triangleleft \gamma) \otimes v_{3} v_{2} v_{1} v_{4} \\
& +(\alpha \rightarrow \beta) \leftarrow \gamma \cdot(\alpha \triangleright \beta) \rightarrow((\alpha \rightarrow \beta) \triangleleft \gamma) \cdot(\alpha \triangleright \beta) \triangleright((\alpha \rightarrow \beta) \triangleleft \gamma) \otimes v_{3} v_{2} v_{4} v_{1}, \\
& \left(v_{1} \prec_{\alpha \triangleleft \beta} v_{2}\right)>_{\alpha \leftarrow \beta} \gamma \otimes v_{3} v_{4} \\
& =(\alpha \leftarrow \beta) \rightarrow \gamma \cdot(\alpha \leftarrow \beta) \triangleright \gamma \cdot \alpha \triangleright \beta \otimes v_{3} v_{4} v_{1} v_{2} \\
& +(\alpha \leftarrow \beta) \rightarrow \gamma \cdot(\alpha \triangleleft \beta) \leftarrow((\alpha \leftarrow \beta) \triangleleft \gamma) \cdot(\alpha \triangleleft \beta) \triangleleft((\alpha \leftarrow \beta) \triangleleft \gamma) \otimes v_{3} v_{1} v_{2} v_{4} \\
& +(\alpha \leftarrow \beta) \rightarrow \gamma \cdot(\alpha \triangleleft \beta) \rightarrow((\alpha \leftarrow \beta) \triangleleft \gamma) \cdot(\alpha \triangleleft \beta) \triangleright((\alpha \leftarrow \beta) \triangleleft \gamma) \otimes v_{3} v_{1} v_{4} v_{2} .
\end{aligned}
$$

As the family $\left(v_{\sigma(1)} v_{\sigma(2)} v_{\sigma(3)} v_{\sigma(4)}\right)_{\sigma \in \mathfrak{S}_{4}}$ is linearly independent, identifying in (43), we obtain (1)-(13).

2. $\Longrightarrow 1$. Let us prove (41)-(43) for $x, y, z$ typed words by induction on the total length $N$ of $x, y$ and $z$. If $x=1$, then (41) is trivially satisfied; If $y=1$, then (42) is trivially satisfied; if $z=1$, then (43) is trivially satisfied. This proves the result if $N \leqslant 2$. We now suppose that $x, y, z \neq 1$ and let us assume the result at all ranks $<N$. Let us put $z=\gamma \otimes v \cdot z^{\prime}$. Using the induction hypothesis:

$$
\begin{aligned}
x>_{\alpha}\left(y>_{\beta} z\right) & =\alpha \rightarrow(\beta \rightarrow \gamma) \otimes v \cdot\left(x>_{\alpha \triangleright(\beta \rightarrow \gamma)}\left(y>_{\beta \triangleright \gamma} z^{\prime}\right)\right) \\
& +\alpha \leftarrow(\beta \rightarrow \gamma) \otimes v \cdot\left(x<_{\alpha \triangleleft(\beta \rightarrow \gamma)}\left(y>_{\beta \triangleright \gamma} z^{\prime}\right)\right) \\
& +\alpha \rightarrow(\beta \leftarrow \gamma) \otimes v \cdot\left(x>_{\alpha \triangleright(\beta \leftarrow \gamma)}\left(y<_{\beta \triangleleft \gamma} z^{\prime}\right)\right) \\
& +\alpha \leftarrow(\beta \leftarrow \gamma) \otimes v \cdot\left(x<_{\alpha \triangleleft(\beta \leftarrow \gamma)}\left(y<_{\beta \triangleleft \gamma} z^{\prime}\right)\right) \\
& =\alpha \rightarrow(\beta \rightarrow \gamma) \otimes v \cdot\left(x>_{(\alpha \triangleright(\beta \rightarrow \gamma)) \triangleright(\beta \triangleleft \gamma)} y\right)>_{(\alpha \triangleright(\beta \rightarrow \gamma)) \rightarrow(\beta \triangleleft \gamma)} z^{\prime} \\
& +\alpha \rightarrow(\beta \rightarrow \gamma) \otimes v \cdot\left(x<_{(\alpha \triangleright(\beta \rightarrow \gamma)) \triangleleft(\beta \triangleleft \gamma)} y\right)>_{(\alpha \triangleright(\beta \rightarrow \gamma)) \leftarrow(\beta \triangleleft \gamma)} z^{\prime} \\
& +\alpha \rightarrow(\beta \leftarrow \gamma) \otimes v \cdot\left(x>_{\alpha \triangleright(\beta \leftarrow \gamma)} y\right)<_{\beta \leftarrow \gamma} z^{\prime} \\
& +\alpha \leftarrow(\beta \leftarrow \gamma) \otimes v \cdot x<_{\alpha \triangleleft(\beta \leftarrow \gamma)}\left(y<_{\beta \triangleleft \gamma} z^{\prime}\right) \\
& +\alpha \leftarrow(\beta \rightarrow \gamma) \otimes v \cdot x<_{\alpha \triangleleft(\beta \rightarrow \gamma)}\left(y>_{\beta \triangleright \gamma} z^{\prime}\right) .
\end{aligned}
$$


Moreover:

$$
\begin{aligned}
\left(x>_{\alpha \triangleright \beta} y\right)>_{\alpha \rightarrow \beta} z & =(\alpha \rightarrow \beta) \rightarrow \gamma \otimes v \cdot\left(x>_{\alpha \triangleright \beta} y\right)>_{(\alpha \rightarrow \beta) \triangleright \gamma} z^{\prime} \\
& +(\alpha \rightarrow \beta) \leftarrow \gamma \otimes v \cdot\left(x>_{\alpha \triangleright \beta} y\right) \prec_{(\alpha \rightarrow \beta) \triangleleft \gamma} z^{\prime} ; \\
\left(x \prec_{\alpha \triangleleft \beta} y\right)>_{\alpha \leftarrow \beta} z & =(\alpha \leftarrow \beta) \rightarrow \gamma \otimes v \cdot\left(x<_{\alpha \triangleleft \beta} y\right)>_{(\alpha \leftarrow \beta) \triangleright \gamma} z^{\prime} \\
& +(\alpha \leftarrow \beta) \leftarrow \gamma \otimes v \cdot\left(x<_{\alpha \triangleleft \beta} y\right) \prec_{(\alpha \leftarrow \beta) \triangleleft \gamma} z^{\prime} \\
& =(\alpha \leftarrow \beta) \rightarrow \gamma \otimes v \cdot\left(x<_{\alpha \triangleleft \beta} y\right)>_{(\alpha \leftarrow \beta) \triangleright \gamma} z^{\prime} \\
& +(\alpha \leftarrow \beta) \leftarrow \gamma \otimes v \cdot x \prec_{(\alpha \triangleleft \beta) \leftarrow((\alpha \leftarrow \beta) \triangleleft \gamma)}\left(y \prec_{(\alpha \triangleleft \beta) \triangleleft((\alpha \leftarrow \beta) \triangleleft \gamma)} z^{\prime}\right) \\
& +(\alpha \leftarrow \beta) \leftarrow \gamma \otimes v \cdot x \prec_{(\alpha \triangleleft \beta) \rightarrow((\alpha \leftarrow \beta) \triangleleft \gamma)}\left(y>_{(\alpha \triangleleft \beta) \triangleright((\alpha \leftarrow \beta) \triangleleft \gamma)} z^{\prime}\right) .
\end{aligned}
$$

With (11)-(13), we conclude that (43) is satisfied for $x, y, z$. Relations (41) and (42) are proved in the same way.

Observe that:

$$
\begin{aligned}
& 1 \prec_{\alpha}^{o p} \alpha_{2} \ldots \alpha_{m} \otimes v_{1} \ldots v_{m}=\alpha_{2} \ldots \alpha_{m} \otimes v_{1} \ldots v_{m}>_{\alpha}^{o p} 1=0, \\
& \alpha_{2} \ldots \alpha_{m} \otimes v_{1} \ldots v_{m} \prec_{\alpha}^{o p} 1=1>_{\alpha}^{o p} \alpha_{2} \ldots \alpha_{n} \otimes v_{1} \ldots v_{m}=\alpha_{2} \ldots \alpha_{m} \otimes v_{1} \ldots v_{m},
\end{aligned}
$$

and

$$
\begin{aligned}
& \alpha_{2} \ldots \alpha_{m} \otimes v_{1} \ldots v_{m} \prec_{\alpha}^{o p} \beta_{2} \ldots \beta_{n} \otimes w_{1} \ldots w_{n} \\
& =\left(\left(\alpha \leftarrow \alpha_{2}\right) \otimes v_{1}\right) \cdot\left(\alpha_{3} \ldots \alpha_{m} \otimes v_{2} \ldots v_{m} \prec_{\alpha_{2} \triangleleft \alpha}^{o p} \beta_{2} \ldots \beta_{n} \otimes w_{1} \ldots w_{n}\right) \\
& +\left(\left(\alpha \rightarrow \alpha_{2}\right) \otimes v_{1}\right) \cdot\left(\alpha_{3} \ldots \alpha_{m} \otimes v_{2} \ldots v_{m}>_{\alpha_{2} \triangleright \alpha}^{o p} \beta_{2} \ldots \beta_{n} \otimes w_{1} \ldots w_{n}\right) \\
& =\left(\left(\alpha_{2} \rightarrow^{o p} \alpha\right) \otimes v_{1}\right) \cdot\left(\alpha_{3} \ldots \alpha_{m} \otimes v_{2} \ldots v_{m} \prec_{\alpha_{2} \triangleleft \alpha}^{o p} \beta_{2} \ldots \beta_{n} \otimes w_{1} \ldots w_{n}\right) \\
& +\left(\left(\alpha_{2} \leftarrow^{o p} \alpha\right) \otimes v_{1}\right) \cdot\left(\alpha_{3} \ldots \alpha_{m} \otimes v_{2} \ldots v_{m}>_{\alpha_{2} \triangleright \alpha}^{o p} \beta_{2} \ldots \beta_{n} \otimes w_{1} \ldots w_{n}\right), \\
& \alpha_{2} \ldots \alpha_{m} \otimes v_{1} \ldots v_{m}>_{\alpha}^{o p} \beta_{2} \ldots \beta_{n} \otimes w_{1} \ldots w_{n} \\
& =\left(\left(\beta_{2} \leftarrow \alpha\right) \otimes w_{1}\right) \cdot\left(\alpha_{2} \ldots \alpha_{m} \otimes v_{1} \ldots v_{m}<_{\alpha \triangleleft \beta_{2}}^{o p} \beta_{3} \ldots \beta_{n} \otimes w_{2} \ldots w_{n}\right) \\
& +\left(\left(\beta_{2} \rightarrow \alpha\right) \otimes w_{1}\right) \cdot\left(\alpha_{2} \ldots \alpha_{m} \otimes v_{1} \ldots v_{m}>_{\alpha \triangleright \beta_{2}}^{o p} \beta_{3} \ldots \beta_{n} \otimes w_{2} \ldots w_{n}\right) \\
& =\left(\left(\alpha \rightarrow{ }^{o p} \beta_{2}\right) \otimes w_{1}\right) \cdot\left(\alpha_{2} \ldots \alpha_{m} \otimes v_{1} \ldots v_{m} \prec_{\alpha \triangleleft \beta_{2}}^{o p} \beta_{3} \ldots \beta_{n} \otimes w_{2} \ldots w_{n}\right) \\
& +\left(\left(\alpha \leftarrow^{o p} \beta_{2}\right) \otimes w_{1}\right) \cdot\left(\alpha_{2} \ldots \alpha_{m} \otimes v_{1} \ldots v_{m}>_{\alpha \triangleright \beta_{2}}^{o p} \beta_{3} \ldots \beta_{n} \otimes w_{2} \ldots w_{n}\right),
\end{aligned}
$$

so

$$
\operatorname{Sh}_{\Omega}^{+}(V)^{o p}=\operatorname{Sh}_{\Omega^{o p}}^{+}(V) .
$$

In particular, if $\Omega$ is commutative, the $\Omega$-dendriform algebra $\operatorname{Sh}_{\Omega}^{+}(V)$ is commutative.

Let us assume that $\Omega$ is commutative. Let $A$ be a commutative $\Omega$-dendriform algebra and let $\phi: V \longrightarrow A$ be any linear map. Let us prove that there exists a unique map $\Phi: \operatorname{Sh}_{\Omega}^{+}(V) \longrightarrow A$ of $\Omega$-dendriform algebras such that $\Phi_{\mid V}=\phi$.

Existence. We inductively define $\Phi$ by:

$$
\begin{aligned}
\Phi(v) & =\phi(v), \\
\Phi\left(\alpha_{2} \ldots \alpha_{n} \otimes v_{1} \ldots v_{n}\right) & =\phi\left(v_{1}\right) \prec_{\alpha_{2}} \Phi\left(\alpha_{3} \ldots \alpha_{n} \otimes v_{2} \ldots v_{n}\right) \text { if } n \geqslant 2 .
\end{aligned}
$$

Let us prove that $\Phi\left(x \prec_{\alpha} y\right)=\Phi(x) \prec_{\alpha} \Phi(y)$ for any typed words $x$ and $y$ by induction on the total length $N$ of $x$ and $y$. If the length of $x$ is 1 :

$$
\Phi\left(x \prec_{\alpha} y\right)=\Phi(\alpha \otimes x \cdot y)=\phi(x) \prec_{\alpha} \Phi(y)=\Phi(x) \prec_{\alpha} \Phi(y) .
$$


This proves the result if $N=2$. Let us assume the result at all ranks $<N$. We can restrict ourselves to the case where the length of $x$ is not 1 . We put $x=(\beta \otimes v) \cdot x^{\prime}$, with $v \in V$ and $x^{\prime}$ is a typed word. Then:

$$
\begin{aligned}
& \Phi\left(x \prec_{\alpha} y\right)=\Phi\left(\beta \leftarrow \alpha \otimes v \cdot x^{\prime} \prec_{\alpha_{\triangleleft} \beta} y\right)+\Phi\left(\beta \rightarrow \alpha \otimes v \cdot x^{\prime}>_{\alpha_{\triangleright} \beta} y\right) \\
& =\phi(v)<_{\beta \leftarrow \alpha} \Phi\left(x^{\prime} \prec_{\alpha_{\triangleleft} \beta} y\right)+\phi(v)<_{\beta \rightarrow \alpha} \Phi\left(x^{\prime}>_{\alpha_{\triangleright} \beta} y\right) \\
& =\phi(v)<_{\beta \leftarrow \alpha} \Phi\left(x^{\prime}<_{\alpha \triangleleft \beta} y\right)+\phi(v)<_{\beta \rightarrow \alpha} \Phi\left(y<_{\alpha \triangleright \beta} x^{\prime}\right) \\
& =\phi(v)<_{\beta \leftarrow \alpha}\left(\Phi\left(x^{\prime}\right)<_{\alpha_{\triangleleft} \beta} \Phi(y)\right)+\phi(v)<_{\beta \rightarrow \alpha}\left(\Phi(y)<_{\alpha_{\triangleright} \beta} \Phi\left(x^{\prime}\right)\right) \\
& =\phi(v)<_{\beta \leftarrow \alpha}\left(\Phi\left(x^{\prime}\right) \prec_{\alpha \triangleleft \beta} \Phi(y)\right)+\phi(v)<_{\beta \rightarrow \alpha}\left(\Phi\left(x^{\prime}\right)>_{\alpha_{\triangleright} \beta} \Phi(y)\right) \\
& =\left(\phi(v)<_{\beta} \Phi\left(x^{\prime}\right)\right)<_{\alpha} \Phi(y) \\
& =\Phi(x) \prec_{\alpha} \Phi(y) \text {. }
\end{aligned}
$$

So $\Phi$ is compatible with $\prec_{\alpha}$. As $A$ and $\operatorname{Sh}_{\Omega}^{+}(V)$ are commutative, for any $x, y \in \operatorname{Sh}_{\Omega}^{+}(V)$,

$$
\Phi\left(x>_{\alpha} y\right)=\Phi\left(y \prec_{\alpha} x\right)=\Phi(y) \prec_{\alpha} \Phi(x)=\Phi(x)>_{\alpha} \Phi(y) .
$$

So $\Phi$ is a morphism of $\Omega$-dendriform algebras.

Unicity. Let $\Psi$ be such a morphism. Then for any typed word $x=(\alpha \otimes v) \cdot x^{\prime}$ of length $\geqslant 2$ :

$$
\Psi(x)=\Psi\left(v \prec_{\alpha} x^{\prime}\right)=\phi(v) \prec_{\alpha} \Psi\left(x^{\prime}\right) .
$$

Hence, $\Psi=\Phi$.

\subsection{From $\Omega$-dendrifrom algebras to dendriform algebras}

Proposition 18. Let $\Omega$ be an EDS and let $A$ be a vector space equipped with bilinear products $<_{\alpha}$ and $>_{\alpha}$. We equip $\mathbb{K} \Omega \otimes A$ with two bilinear products $<,>$ defined in the following way:

$$
\begin{aligned}
\forall \alpha, \beta \in \Omega, \forall x, y \in A, & \alpha \otimes x<\beta \otimes y=\alpha \leftarrow \beta \otimes x<_{\alpha \triangleleft \beta} y, \\
\alpha \otimes x>\beta \otimes y & =\alpha \rightarrow \beta \otimes x>_{\alpha \triangleright \beta} y .
\end{aligned}
$$

1. If $\left(A,\left(<_{\alpha}\right)_{\alpha \in \Omega},\left(>_{\alpha}\right)_{\alpha \in \Omega}\right)$ is $\Omega$-dendriform, then $(\mathbb{K} \Omega \otimes A,<,>)$ is dendriform.

2. If $\varphi_{\leftarrow}$ and $\varphi_{\rightarrow}$ are surjective, then the converse implication is true.

Proof. Let $\alpha, \beta, \gamma \in \Omega$ and $x, y, z \in A$.

$$
\begin{aligned}
(\alpha \otimes x<\beta \otimes y)<\gamma \otimes z & =(\alpha \rightarrow \beta) \rightarrow \gamma \otimes\left(x<_{\alpha \triangleleft \beta} y\right)<_{(\alpha \leftarrow \beta) \triangleleft \gamma} z, \\
\alpha \otimes x<(\beta \otimes y<\gamma \otimes z+\beta \otimes y>\gamma \otimes z) & =\alpha \leftarrow(\beta \leftarrow \gamma) \otimes x<_{\alpha \triangleleft(\beta \leftarrow \gamma)}\left(y<_{\beta \triangleleft \gamma} z\right) \\
& +\alpha \leftarrow(\beta \rightarrow \gamma) \otimes x<_{\alpha \triangleleft(\beta \rightarrow \gamma)}\left(y>_{\beta \triangleright \gamma} z\right) .
\end{aligned}
$$

As $(\Omega, \leftarrow, \rightarrow)$ is diassociative,

$$
(\alpha \rightarrow \beta) \rightarrow \gamma=\alpha \leftarrow(\beta \leftarrow \gamma)=\alpha \leftarrow(\beta \rightarrow \gamma) .
$$

1. Let us assume that $A$ is $\Omega$-dendriform. Then, as $\Omega$ is an EDS:

$$
\begin{aligned}
\left(x<_{\alpha \triangleleft \beta} y\right)<_{(\alpha \leftarrow \beta) \triangleleft \gamma} z & =x<_{(\alpha \triangleleft \beta) \leftarrow((\alpha \leftarrow \beta) \triangleleft \gamma)}\left(y<_{(\alpha \triangleleft \beta) \triangleleft((\alpha \leftarrow \beta) \triangleleft \gamma)} z\right) \\
& +x \prec_{(\alpha \triangleleft \beta) \rightarrow((\alpha \leftarrow \beta) \triangleleft \gamma)}\left(y>_{(\alpha \triangleleft \beta) \triangleright(((\alpha \leftarrow \beta) \triangleleft \gamma)} z\right) \\
& =x \prec_{\alpha \triangleleft(\beta \leftarrow \gamma)}\left(y \prec_{\beta \triangleleft \gamma} z\right)+x \prec_{\alpha \triangleleft(\beta \rightarrow \gamma)}\left(y>_{\beta \triangleright \gamma} z\right) .
\end{aligned}
$$

So the first dendriform relation is satisfied. The second and third ones are proved in the same way. 
2. Let us assume that $\mathbb{K} \Omega \otimes A$ is dendriform and that $\varphi_{\leftarrow}$ and $\varphi_{\rightarrow}$ are surjective. For any $x, y, z \in A$, for any $\alpha, \beta, \gamma \in \Omega$ :

$$
\left(x \prec_{\alpha \triangleleft \beta} y\right) \prec_{(\alpha \leftarrow \beta) \triangleleft \gamma} z=x \prec_{\alpha \triangleleft(\beta \leftarrow \gamma)}\left(y \prec_{\beta \triangleleft \gamma} z\right)+x \prec_{\alpha \triangleleft(\beta \rightarrow \gamma)}\left(y>_{\beta \triangleright \gamma} z\right) .
$$

By hypothesis, the following map is surjective:

$$
\varphi_{\leftarrow}^{\prime}:\left\{\begin{array}{rll}
\Omega^{2} & \longrightarrow \Omega^{2} \\
(\alpha, \beta) & \longrightarrow & (\alpha \triangleleft, \alpha \leftarrow \beta) .
\end{array}\right.
$$

By composition, the following map is surjective:

$$
\left(I d \otimes \varphi_{\leftarrow}^{\prime}\right) \circ\left(\varphi_{\leftarrow}^{\prime} \otimes I d\right):\left\{\begin{aligned}
\Omega^{3} & \longrightarrow \Omega^{3} \\
(\alpha, \beta, \gamma) & \longrightarrow(\alpha \triangleleft \beta,(\alpha \leftarrow \beta) \triangleleft \gamma, \alpha \leftarrow \beta \leftarrow \gamma) .
\end{aligned}\right.
$$

Let $\left(\alpha^{\prime}, \beta^{\prime}, \gamma^{\prime}\right) \in \Omega^{3}$ and let $(\alpha, \beta, \gamma) \in \Omega^{3}$ such that:

$$
\alpha^{\prime}=\alpha \triangleleft \beta, \quad \quad \beta^{\prime}=(\alpha \rightarrow \beta) \triangleleft \gamma, \quad \gamma^{\prime}=\alpha \leftarrow \beta \leftarrow \gamma .
$$

Then, by (6)- (9):

$$
\begin{aligned}
\left(x<_{\alpha^{\prime}} y\right) \prec_{\beta^{\prime}} z & =x \prec_{\alpha \triangleleft(\beta \leftarrow \gamma)}\left(y<_{\beta \triangleleft \gamma} z\right)+x<_{\alpha \triangleleft(\beta \rightarrow \gamma)}\left(y>_{\beta \triangleright \gamma} z\right) \\
& =x \prec_{\alpha^{\prime} \leftarrow \beta^{\prime}}\left(y<_{\alpha^{\prime} \triangleleft \beta^{\prime}} z\right)+x \prec_{\alpha^{\prime} \rightarrow \beta^{\prime}}\left(y>_{\alpha^{\prime} \triangleright \beta^{\prime}} z\right)
\end{aligned}
$$

So the first $\Omega$-dendriform relation is satisfied. The two other ones are similarly proved.

Let us now study the dendriform algebras $\mathbb{K} \Omega \otimes \mathbb{K} \mathcal{T}_{\Omega}^{+}$and $\mathbb{K} \Omega \otimes \operatorname{Sh}_{\Omega}^{+}(V)$.

Proposition 19. Let $\Omega$ be an EDS.

1. The following assertions are equivalent:

(a) The dendriform algebra $\mathbb{K} \Omega \otimes \mathbb{K} \mathcal{T}_{\Omega}^{+}$is generated by the elements $\alpha \otimes Y, \alpha \in \Omega$.

(b) $\varphi_{\leftarrow}$ and $\varphi_{\rightarrow}$ are surjective.

2. The following assertions are equivalent:

(a) The dendriform subalgebra of $\mathbb{K} \Omega \otimes \mathbb{K} \mathcal{T}_{\Omega}^{+}$generated by the elements $\alpha \otimes Y, \alpha \in \Omega$, is free.

(b) $\varphi_{\leftarrow}$ and $\varphi_{\rightarrow}$ are injective.

Proof. Firstly, observe that the dendriform algebra $\mathbb{K} \Omega \otimes \mathcal{T}_{\Omega}^{+}$is graded, with for any $n \geqslant 1$,

$$
\left(\mathbb{K} \Omega \otimes \mathcal{T}_{\Omega}^{+}\right)(n)=\mathbb{K} \Omega \otimes \mathcal{T}_{\Omega}(n) .
$$

1. $(a) \Longrightarrow(b)$. Let $\alpha, \beta \in \Omega$. As $\mathbb{K} \Omega \otimes \mathcal{T}_{\Omega}^{+}$is graded, there exists families of scalars $\left(\lambda_{a, b}\right)_{a, b \in \Omega}$ and $\left(\mu_{a, b}\right)_{a, b \in \Omega}$ such that:

$$
\begin{aligned}
\alpha \otimes{ }^{Y}(\beta) & =\sum_{a, b \in \Omega} \lambda_{a, b} a \otimes Y<b \otimes Y+\sum_{a, b \in \Omega} \mu_{a, b} a \otimes Y>b \otimes Y \\
& =\sum_{a, b \in \Omega} \lambda_{a, b} a \leftarrow b \otimes Y^{\prime}(a \triangleleft b)+\sum_{a, b \in \Omega} \mu_{a, b} a \rightarrow b \otimes Y_{(a \triangleright b)} .
\end{aligned}
$$

Hence, there exists $(a, b) \in \Omega^{2}$, such that $a \rightarrow b=\alpha$ and $a \triangleright b=\beta: \varphi_{\rightarrow}$ is surjective. Similarly, $\varphi_{\leftarrow}$ is surjective. 
1. $(b) \Longrightarrow(a)$. Let us denote by $A$ the dendriform subalgebra of $\mathbb{K} \Omega \otimes \mathbb{K} \mathcal{T}_{\Omega}^{+}$generated by the elements $\alpha \otimes Y$. Let us prove that for any $\alpha \in \Omega, T \in \mathcal{T}_{\Omega}, \alpha \otimes T \in A$ by induction on the number $N$ of leaves of $T$. If $N=2$, then $T=Y$ and it is obvious. Otherwise, let us put $T=T_{1} \bigvee_{\beta, \gamma} T_{2}$. By the induction hypothesis, for $i=1$ or $2, T_{i}=$ । or $T_{i} \in A$. If $T_{1} \neq$ ।, let $\left(\alpha^{\prime}, \beta^{\prime}\right) \in \Omega$ such that $\varphi_{\rightarrow}\left(\alpha^{\prime}, \beta^{\prime}\right)=(\alpha, \beta)$. Then

$$
\begin{aligned}
\alpha^{\prime} \otimes T_{1}>\beta^{\prime} \otimes \mid \bigvee_{\varnothing, \gamma} T_{2} & =\alpha^{\prime} \rightarrow \beta^{\prime} \otimes T_{1}>_{\alpha^{\prime} \triangleright \beta^{\prime}} \mid \bigvee_{\varnothing, \gamma} T_{2} \\
& =\alpha \otimes T_{1}>_{\beta} \mid \bigvee_{\varnothing, \gamma} T_{2} \\
& =\alpha \otimes T_{1} \bigvee_{\beta, \gamma} T_{2}
\end{aligned}
$$

So $\alpha \otimes T \in A$. Similarly, if $T_{2} \neq \mathrm{I}$, then $\alpha \otimes T \in A$.

2. $(a) \Longrightarrow(b)$. Because of the graduation, $A$ is freely generated by the elements $\alpha \otimes Y$, with $\alpha \in \Omega$. Let $(\alpha, \beta),\left(\alpha^{\prime}, \beta^{\prime}\right) \in \Omega^{2}$, such that $\varphi_{\leftarrow}(\alpha, \beta)=\varphi_{\leftarrow}\left(\alpha^{\prime}, \beta^{\prime}\right)$. Then:

$$
\alpha \otimes Y<\beta \otimes Y=\alpha \leftarrow \beta \otimes Y^{1}(\alpha \triangleleft \beta) \alpha^{\prime} \leftarrow \beta^{\prime} \otimes Y_{1}\left(\alpha^{\prime} \triangleleft \beta^{\prime}\right)=\alpha^{\prime} \otimes Y<\beta^{\prime} \otimes Y .
$$

By freeness of $\mathbb{K} \Omega \otimes \mathbb{K} \mathcal{T}_{\Omega}^{+},(\alpha, \beta)=\left(\alpha^{\prime}, \beta^{\prime}\right)$, so $\varphi_{\leftarrow}$ is injective. The proof is similar for $\varphi_{\rightarrow}$.

2. $(b) \Longrightarrow(a)$. Let Dend $(\Omega)$ be the free dendriform algebra generated by $\Omega$. As a vector space, it is generated by plane binary trees which internal vertices are decorated by $\Omega$. Let $\Theta: \operatorname{Dend}(\Omega) \longrightarrow \mathbb{K} \Omega \otimes \mathbb{K} \mathcal{T}_{\Omega}^{+}$be the unique dendriform algebra morphism sending $\alpha \in \Omega$ to $\alpha \otimes Y$. Then, for any tree $T \in \operatorname{Dend}(\Omega)$, writing it as $T=T_{1} \bigvee_{\alpha} T_{2}, \alpha$ being the decoration of the root of $T$, let us denote

$$
\Theta\left(T_{1}\right)=\sum_{i} \alpha_{i} T_{1}^{(i)}, \quad \Theta\left(T_{2}\right)=\sum_{j} \beta_{j} T_{2}^{(j)}
$$

Then:

$$
\Theta(T)=\sum_{i, j} \alpha_{i} \rightarrow \alpha \leftarrow \beta_{j} \otimes T_{1}^{(i)} \bigvee_{\alpha_{i} \triangleright \alpha,\left(\alpha_{i} \rightarrow \alpha\right) \triangleleft \beta_{j}} T_{2}^{(j)} .
$$

We conclude that $\Theta(T)$ is a typed tree of the same form as $T$, with types of edges obtained from the decorations of the vertices of $T$ by the application of compositions of maps $I d^{\otimes(i-1)} \otimes \varphi_{\leftarrow} \otimes$ $I d^{\otimes(n-i)}$ and $I d^{\otimes(i-1)} \otimes \varphi_{\rightarrow} \otimes I d^{\otimes(n-i)}$. As $\varphi_{\leftarrow}$ and $\varphi_{\rightarrow}$ are injective, $\Theta$ is injective.

Proposition 20. Let $\Omega$ be a commutative EDS and $V$ be a nonzero vector space.

1. The following assertions are equivalent:

(a) The dendriform algebra $\mathbb{K} \Omega \otimes \mathrm{Sh}_{\Omega}^{+}(V)$ is generated by the elements $\alpha \otimes v, \alpha \in \Omega$, $v \in V$.

(b) $\varphi_{\leftarrow}$ is surjective.

2. The following assertions are equivalent:

(a) The commutative dendriform subalgebra of $\mathbb{K} \Omega \otimes \mathrm{Sh}_{\Omega}^{+}(V)$ generated by the elements $\alpha \otimes v, \alpha \in \Omega, v \in V$, is free.

(b) $\varphi_{\leftarrow}$ is injective. 
Proof. 1. $(a) \Longrightarrow(b)$. Let $\alpha, \beta \in \Omega$. Let us choose a nonzero element of $V$. Then $\alpha \otimes(\beta \otimes v)$ belongs to the dendriform subalgebra of $\mathbb{K} \Omega \otimes \operatorname{Sh}_{\Omega}^{+}(V)$. As it is graded, it can be written under the form:

$$
\alpha \otimes(\beta \otimes v v)=\sum_{i} \alpha_{i} \otimes v_{i} \prec \beta_{i} \otimes w_{i}=\sum_{i} \alpha_{i} \leftarrow \beta_{i} \otimes\left(\alpha_{i} \triangleleft \beta_{i} \otimes v_{i} w_{i}\right)
$$

where $v_{i}, w_{i} \in V$ and $\alpha_{i}, \beta_{i} \in \Omega$ for any $i$. Hence, there exists $i$, such that $\varphi_{\leftarrow}\left(\alpha_{i}, \beta_{i}\right)=(\alpha, \beta)$.

1. $(b) \Longrightarrow(a)$. Let us assume that $\varphi_{\leftarrow}$ is surjective. Let us denote by $A$ the dendriform subalgebra of $\mathbb{K} \Omega \otimes \operatorname{Sh}_{\Omega}^{+}(V)$ generated by the elements $\alpha \otimes v$. Let us prove that any typed word $\alpha_{2} \ldots \alpha_{k} \otimes v_{1} \ldots v_{k}$, for any $\alpha_{1} \in \Omega, \alpha_{1} \otimes\left(\alpha_{2} \ldots \alpha_{k} \otimes v_{1} \ldots v_{k}\right)$ belongs to $A$ by induction on $n$. It is obvious if $n=1$. Otherwise, let $\left(\beta_{1}, \beta_{2}\right) \in \Omega^{2}$, such that $\varphi\left(\beta_{1}, \beta_{2}\right)=\left(\alpha_{1}, \alpha_{2}\right)$. Then:

$$
\begin{aligned}
\beta_{1} \otimes v_{1}<\beta_{2} \otimes\left(\alpha_{3} \ldots \alpha_{k} \otimes v_{2} \ldots v_{k}\right) & =\beta_{1} \leftarrow \beta_{2} \otimes\left(v_{1} \prec_{\beta_{1} \triangleleft \beta_{2}} \alpha_{3} \ldots \alpha_{k} \otimes v_{2} \ldots v_{k}\right) \\
& =\alpha_{1} \otimes\left(v_{1} \prec_{\alpha_{2}} \alpha_{3} \ldots \alpha_{k} \otimes v_{2} \ldots v_{k}\right) \\
& =\alpha_{1} \otimes\left(\alpha_{2} \ldots \alpha_{k} \otimes v_{1} \ldots v_{k}\right) .
\end{aligned}
$$

By the induction hypothesis, this belongs to $A$.

2. $(a) \Longrightarrow(b)$. Let $\alpha, \beta, \alpha^{\prime}, \beta^{\prime} \in \Omega$ such that $\varphi_{\leftarrow}(\alpha, \beta)=\varphi_{\leftarrow}\left(\alpha^{\prime}, \beta^{\prime}\right)$. Let $v \in V$, nonzero. Then:

$$
\alpha \otimes v<\beta \otimes v=\alpha \leftarrow \beta \otimes(\alpha \triangleleft \beta \otimes v v)=\alpha^{\prime} \leftarrow \beta^{\prime} \otimes\left(\alpha^{\prime} \triangleleft \beta^{\prime} \otimes v v\right)=\alpha^{\prime} \otimes v<\beta^{\prime} \otimes v .
$$

By freeness of $\mathbb{K} \Omega \otimes \operatorname{Sh}_{\Omega}^{+}(V),(\alpha, \beta)=\left(\alpha^{\prime}, \beta^{\prime}\right)$.

3. $(b) \Longrightarrow(a)$. Recall that the free commutative dendriform algebra generated by $\mathbb{K} \Omega \otimes V$ is the shuffle algebra $\mathrm{Sh}^{+}(\mathbb{K} \Omega \otimes V)$, with the usual half-shuffle product. Hence, there exists a dendriform algebra morphism $\Phi: \mathrm{Sh}^{+}(\mathbb{K} \Omega \otimes V) \longrightarrow \mathbb{K} \Omega \otimes \mathrm{Sh}_{\Omega}^{+}(V)$, sending $\alpha \otimes v$ to itself. For any $\alpha_{1}, \ldots, \alpha_{n} \in \Omega, v_{1}, \ldots, v_{n} \in V$, in $\operatorname{Sh}^{+}(V)$ :

$$
\left(\alpha_{1} \otimes v_{1}\right) \ldots\left(\alpha_{n} \otimes v_{n}\right)=\left(\alpha_{1} \otimes v_{1}\right)<\left(\left(\alpha_{2} \otimes v_{2}\right) \ldots\left(\alpha_{n} \otimes v_{n}\right)\right) .
$$

Hence, an easy induction allows to prove that

$$
\Psi\left(\left(\alpha_{1} \otimes v_{1}\right) \ldots\left(\alpha_{n} \otimes v_{n}\right)\right)=\left(\varphi_{\leftarrow} \otimes I d^{\otimes(n-2)}\right) \circ \ldots \circ\left(I d^{\otimes(n-2)} \otimes \varphi_{\leftarrow}\right)\left(\alpha_{1} \ldots \alpha_{n}\right) \otimes v_{1} \ldots v_{n} .
$$

As $\varphi_{\leftarrow}$ is injective, $\Psi$ is injective.

\section{Operad of $\Omega$-dendriform algebras}

We fix in this section an $\operatorname{EDS}(\Omega, \leftarrow, \rightarrow, \triangleleft, \triangleright)$.

\subsection{Combinatorial description of the operad}

Let us denote by $\mathcal{P}_{\Omega}$ the (nonsymmetric) operad of $\Omega$-dendriform algebras. It is generated by elements $<_{\alpha},>_{\alpha} \in \mathcal{P}(2)$, with $\alpha \in \Omega$, and the relations:

$$
\begin{aligned}
\forall \alpha, \beta \in \Omega, & <_{\beta} \circ\left(<_{\alpha}, I\right)=<_{\alpha \leftarrow \beta} \circ\left(I,<_{\alpha \triangleleft \beta}\right)+<_{\alpha \rightarrow \beta} \circ\left(I,>_{\alpha \triangleright \beta}\right), \\
& >_{\alpha} \circ\left(I,<_{\beta}\right)=<_{\beta} \circ\left(>_{\alpha}, I\right), \\
& >_{\alpha} \circ\left(I,>_{\beta}\right)=>_{\alpha \rightarrow \beta} \circ\left(>_{\alpha \triangleright \beta}, I\right)+>_{\alpha \leftarrow \beta} \circ\left(<_{\alpha \triangleleft \beta}, I\right) .
\end{aligned}
$$


As we know from Proposition $[15$ a combinatorial description of the free $\Omega$-dendriform algebra on one generator, we obtain a combinatorial description of this operad:

$$
\forall n \geqslant 1, \quad \mathcal{P}_{\Omega}(n)=\mathbb{K} \mathcal{T}_{\Omega}(n) .
$$

The composition is given by the actions of the products of $\mathbb{K} \mathcal{T}_{\Omega}^{+}$. In particular:

$$
I=Y, \quad<_{\alpha}=Y<_{\alpha} Y=Y^{1}(\alpha), \quad>_{\alpha}=Y>_{\alpha} Y={ }^{1}(\alpha) .
$$

The operadic composition can be inductively computed with the help of the following formula:

$$
T_{1} \bigvee_{\alpha, \beta} T_{2} \circ\left(T_{1}^{\prime}, \ldots, T_{k}^{\prime}\right)=T_{1} \circ\left(T_{1}^{\prime}, \ldots, T_{i}^{\prime}\right)>_{\alpha} T_{i+1}^{\prime}<_{\beta} T_{2} \circ\left(T_{i+2}^{\prime}, \ldots, T_{k}^{\prime}\right),
$$

where $T_{1}$ is a tree with $i$ internal vertices, $T_{2}$ is a tree with $k-i-1$ internal vertices, and $T_{1}^{\prime}, \ldots, T_{k}^{\prime}$ are trees.

Example 8. Here are examples of operadic compositions:

$$
\begin{aligned}
& \prec_{\alpha} \circ\left(<_{\beta}, I\right)=Y_{1}(\beta)<_{\alpha} Y=Y_{1}{ }^{2}(\beta \leftarrow \alpha, \beta \triangleleft \alpha)+Y^{2} /{ }^{\prime}(\beta \rightarrow \alpha, \beta \triangleright \alpha), \\
& \prec_{\alpha} \circ\left(I,<_{\beta}\right)=Y \prec_{\alpha} Y^{1}(\beta)=Y_{1}{ }^{2}(\alpha, \beta), \\
& >_{\alpha} \circ\left(<_{\beta}, I\right)=Y_{1}(\beta)>_{\alpha} Y=Y^{1 / 2}(\alpha, \beta), \\
& >_{\alpha} \circ\left(I,<_{\beta}\right)=Y>_{\alpha} Y^{1}(\beta)={ }^{1} Y_{2}(\alpha, \beta), \\
& \prec_{\alpha} \circ\left(>_{\beta}, I\right)={ }^{Y} Y(\beta) \prec_{\alpha} Y=Y_{1} Y_{2}(\beta, \alpha), \\
& \prec_{\alpha} \circ\left(I,>_{\beta}\right)=Y \prec_{\alpha} Y_{(\beta)}=Y^{2} /{ }^{\prime}(\alpha, \beta) \text {, } \\
& >_{\alpha} \circ\left(>_{\beta}, I\right)=Y_{1}(\beta)>_{\alpha} Y={ }^{2} Y(\alpha, \beta) \text {, } \\
& >_{\alpha} \circ\left(I,>_{\beta}\right)=Y>_{\alpha} Y_{Y}(\beta)=\stackrel{Y_{2}}{{ }^{\prime}}(\alpha \leftarrow \beta, \alpha \triangleleft \beta)+{ }^{2}{ }_{1}(\alpha \rightarrow \beta, \alpha \triangleright \beta) \text {. }
\end{aligned}
$$

\subsection{Associative products}

Proposition 21. Let $m \in \mathcal{P}_{\Omega}(2)$, written under the form

$$
m=\sum_{\alpha \in \Omega} a_{\alpha}<_{\alpha}+\sum_{\alpha \in \Omega} b_{\alpha}>_{\alpha} .
$$

Then $m \circ(I, m)=m \circ(m, I)$ if, and only if, for any $\alpha, \beta \in \Omega$ :

$$
\begin{aligned}
b_{\alpha} b_{\beta} & =\sum_{\varphi \rightarrow\left(\alpha^{\prime}, \beta^{\prime}\right)=(\alpha, \beta)} b_{\alpha^{\prime}} b_{\beta^{\prime}}, & a_{\alpha} a_{\beta} & =\sum_{\varphi \leftarrow\left(\alpha^{\prime}, \beta^{\prime}\right)=(\alpha, \beta)} a_{\alpha^{\prime}} a_{\beta^{\prime}}, \\
b_{\alpha} a_{\beta} & =\sum_{\varphi \leftarrow\left(\alpha^{\prime}, \beta^{\prime}\right)=(\alpha, \beta)} b_{\alpha^{\prime}} b_{\beta^{\prime}}, & a_{\alpha} b_{\beta} & =\sum_{\varphi \rightarrow\left(\alpha^{\prime}, \beta^{\prime}\right)=(\alpha, \beta)} a_{\alpha^{\prime}} a_{\beta^{\prime}} .
\end{aligned}
$$


Proof. Indeed:

$$
\begin{aligned}
& m \circ(I, m)=\sum_{\alpha, \beta \in \Omega} a_{\alpha} a_{\beta} Y^{Y / 2}(\alpha, \beta)+\sum_{\alpha, \beta \in \Omega} a_{\alpha} b_{\beta} \stackrel{2}{1} / 1_{(\alpha, \beta)} \\
& +\sum_{\alpha, \beta \in \Omega} b_{\alpha} b_{\beta}\left(\stackrel{2^{Y}}{1} Y(\alpha \rightarrow \beta, \alpha \triangleright \beta)+{ }^{1 / 2}(\alpha \leftarrow \beta, \alpha \triangleleft \beta)\right)+\sum_{\alpha, \beta \in \Omega} b_{\alpha} a_{\beta}{ }^{1} Y_{2}(\alpha, \beta), \\
& m \circ(m, I)=\sum_{\alpha, \beta \in \Omega} a_{\alpha} a_{\beta}\left(Y^{2 / 1}(\alpha \rightarrow \beta, \alpha \triangleright \beta)+Y_{1}^{Y / 2}(\alpha \leftarrow \beta, \alpha \triangleleft \beta)\right)+\sum_{\alpha, \beta \in \Omega} a_{\alpha} b_{\beta}{ }^{Y Y_{2}}(\beta, \alpha) \\
& +\sum_{\alpha, \beta \in \Omega} b_{\alpha} a_{\beta} \stackrel{Y}{1} Y^{2}(\alpha, \beta)+\sum_{\alpha, \beta \in \Omega} b_{\alpha} b_{\beta}{ }^{2} Y_{Y}(\alpha, \beta) .
\end{aligned}
$$

Identifying, we obtain the announced equations.

Corollary 22. If $\Omega$ is nondegenerate, then $m \circ(I, m)=m \circ(m, I)$ if, and only if, for any $\alpha, \beta \in \Omega$ :

$$
\begin{aligned}
b_{\alpha \rightarrow \beta} b_{\alpha \triangleright \beta} & =b_{\alpha} b_{\beta}, & a_{\alpha \leftarrow \beta} a_{\alpha \triangleleft \beta} & =a_{\alpha} a_{\beta}, \\
b_{\alpha \leftarrow \beta} a_{\alpha \triangleleft \beta} & =b_{\alpha} b_{\beta}, & a_{\alpha \rightarrow \beta} b_{\alpha \triangleright \beta} & =a_{\alpha} a_{\beta} .
\end{aligned}
$$

In particular cases of EDS:

Proposition 23. 1. Let $(\Omega, \star)$ be a group. In $\mathcal{P}_{\mathbf{E D S}(\Omega, \star, \star)}$, the nonzero associative products are of the form

$$
\lambda \sum_{\alpha \in G} \prec_{\alpha}+>_{\alpha}
$$

where $\lambda$ is a nonzero scalar and $G$ is a subgroup of $(\Omega, \star)$.

2. Let $(H, \star)$ be a group, $K$ be a nonempty set and $\theta: K \longrightarrow H$ be a map. In $\mathcal{P}_{\operatorname{EDS}^{*}(H, \star, K, \theta)}$, the nonzero associative products are of the form

$$
\sum_{\alpha^{\prime} \in K} \lambda_{\alpha^{\prime}}\left(\sum_{\alpha \in G} \prec_{\left(\alpha, \alpha^{\prime}\right)}+>_{\left(\theta\left(\alpha^{\prime}\right) \star \alpha, \alpha^{\prime}\right)}\right),
$$

where $\left(\lambda_{\alpha^{\prime}}\right)_{\alpha^{\prime} \in K}$ is a nonzero family of scalars with finite support and $G$ is a subgroup of $(H, \star)$.

Proof. 1. In this case, 45 becomes:

$$
\begin{array}{rlrl}
b_{\alpha \star \beta} b_{\alpha} & =b_{\alpha} b_{\beta}, & a_{\alpha \star \beta} a_{\beta}=a_{\alpha} a_{\beta}, \\
b_{\alpha \star \beta} b_{\beta}=b_{\alpha} a_{\beta}, & a_{\alpha \star \beta} a_{\alpha}=a_{\alpha} b_{\beta} .
\end{array}
$$

We put $G_{a}=\left\{\alpha \in \Omega, a_{\alpha} \neq 0\right\}$ and $G_{b}=\left\{\alpha \in \Omega, b_{\alpha} \neq 0\right\}$. At least one of them is not empty: let us assume for example that $G_{b} \neq \varnothing$. Let $\alpha \in G_{b}$. If $\beta=e$ is the unit of $\Omega$ :

$$
b_{\alpha \star e} b_{\alpha}=b_{\alpha}^{2}=b_{\alpha} b_{e}
$$

so $b_{e}=b_{\alpha} \neq 0: e \in G_{b}$ and for any $\alpha \in G_{b}, b_{\alpha}=b_{e}$. If $\alpha, \beta \in G_{b}$, then:

$$
b_{\alpha \star \beta} b_{\alpha}=b_{\alpha} b_{\beta} \neq 0,
$$

so $\alpha \star \beta \in G_{b}$. For any $\alpha \in G_{b}$, if $\beta=\alpha^{-1}$ :

$$
b_{e} b_{\alpha}=b_{\alpha} b_{\alpha^{-1}} \neq 0
$$


so $\alpha^{-1} \in G_{b}$ : we proved that $G_{b}$ is a subgroup of $\Omega$. If $\beta \in G_{a}$, then

$$
a_{e} b_{\beta}=a_{e \star \beta} a_{\beta}=a_{\beta}^{2} \neq 0,
$$

so $\beta \in G_{b}: G_{a} \subseteq G_{b}$. Conversely, if $\beta \in G_{b}$,

$$
b_{e} a_{\beta}=b_{e \star \beta} b_{\beta}=b_{\beta}^{2} \neq 0,
$$

so $a_{\beta} \neq 0$ : $G_{b} \subseteq G_{a}$. Moreover, as $b_{e}=b_{\beta}$ for any $\beta \in \Omega$, we obtain that $a_{\beta}=b_{e}$. Putting $\lambda=b_{\beta}$ and $G=G_{a}=G_{b}$, we obtain that

$$
m=\lambda \sum_{\alpha \in G} \prec_{\alpha}+>_{\alpha} .
$$

Conversely, for such a $m$, 45 is satisfied, so $m$ is associative.

2. In this case, 45 becomes:

$$
\begin{aligned}
b_{\left(\beta, \beta^{\prime}\right)} b_{\left(\theta\left(\beta^{\prime}\right) \star \beta^{-1} \star \alpha, \alpha^{\prime}\right)} & =b_{\left(\alpha, \alpha^{\prime}\right)} b_{\left(\beta, \beta^{\prime}\right)}, \\
a_{\left(\alpha, \alpha^{\prime}\right)} a_{\left(\alpha^{-1} \star \beta, \beta^{\prime}\right)} & =a_{\left(\alpha, \alpha^{\prime}\right)} a_{\left(\beta, \beta^{\prime}\right)}, \\
b_{\left(\alpha, \alpha^{\prime}\right)} a_{\left(\alpha^{-1} \star \beta, \beta^{\prime}\right)} & =b_{\left(\alpha, \alpha^{\prime}\right)} b_{\left(\beta, \beta^{\prime}\right)}, \\
a_{\left(\beta, \beta^{\prime}\right)} b_{\left(\theta\left(\beta^{\prime}\right) \star \beta^{-1} \star \alpha, \alpha^{\prime}\right)} & =a_{\left(\alpha, \alpha^{\prime}\right)} a_{\left(\beta, \beta^{\prime}\right)} .
\end{aligned}
$$

For any $\alpha^{\prime} \in K$, we put:

$$
G_{a}\left(\alpha^{\prime}\right)=\left\{\alpha \in H, a_{\left(\alpha, \alpha^{\prime}\right)} \neq 0\right\}, \quad G_{b}\left(\alpha^{\prime}\right)=\left\{\alpha \in H, b_{\left(\theta\left(\alpha^{\prime}\right) \star \alpha, \alpha^{\prime}\right)} \neq 0\right\} .
$$

Let $\alpha^{\prime} \in K$, such that $G_{a}\left(\alpha^{\prime}\right) \neq \varnothing$. For any $\alpha, \beta \in G_{a}\left(\alpha^{\prime}\right)$ :

$$
a_{\left(\alpha, \alpha^{\prime}\right)} a_{\left(\beta, \alpha^{\prime}\right)}=a_{\left(\alpha, \alpha^{\prime}\right)} a_{\left(\alpha^{-1} \star \beta, \alpha^{\prime}\right)}, \quad a_{\left(\beta, \alpha^{\prime}\right)}=a_{\left(\alpha^{-1} \star \beta, \alpha^{\prime}\right)} .
$$

So $\alpha^{-1} \star \beta \in G_{a}\left(\alpha^{\prime}\right)$. Hence, $G_{a}\left(\alpha^{\prime}\right)$ is a subgroup of $H$. Moreover, there exists a nonzero scalar $a_{\alpha^{\prime}}$ such that for any $\alpha \in G_{a}\left(\alpha^{\prime}\right), a_{\left(\alpha, \alpha^{\prime}\right)}=a_{\alpha^{\prime}}$.

Let $\alpha^{\prime} \in K$, such that $G_{b}\left(\alpha^{\prime}\right) \neq \varnothing$. For any $\alpha, \beta \in G_{b}\left(\alpha^{\prime}\right)$ :

$$
\begin{aligned}
b_{\left(\theta\left(\alpha^{\prime}\right) \star \alpha, \alpha^{\prime}\right)} b_{\left(\theta\left(\alpha^{\prime}\right) \star \beta, \alpha^{\prime}\right)} & =b_{\left(\theta\left(\alpha^{\prime}\right) \star \beta^{-1} \star \theta\left(\alpha^{\prime}\right)^{-1} \star \theta\left(\alpha^{\prime}\right) \star \alpha, \alpha^{\prime}\right)} b_{\left(\theta\left(\alpha^{\prime}\right) \star \beta, \alpha^{\prime}\right)}, \\
b_{\left(\theta\left(\alpha^{\prime}\right) \star \alpha, \alpha^{\prime}\right)} & =b_{\left(\theta\left(\alpha^{\prime}\right) \star \beta^{-1} \star \alpha, \alpha^{\prime}\right)} .
\end{aligned}
$$

So $\beta^{-1} \star \alpha \in G_{b}\left(\alpha^{\prime}\right)$. Hence, $G_{b}\left(\alpha^{\prime}\right)$ is a subgroup of $H$. Moreover, there exists a nonzero scalar $b_{\alpha^{\prime}}$ such that for any $\alpha \in G_{b}\left(\alpha^{\prime}\right), b_{\left(\theta\left(\alpha^{\prime}\right) \star \alpha, \alpha^{\prime}\right)}=b_{\alpha^{\prime}}$.

Let $\alpha \in G_{a}\left(\alpha^{\prime}\right)$. Then $G_{a}\left(\alpha^{\prime}\right) \neq \varnothing$, so is a subgroup of $H$, and the unit $e$ of $H$ belongs to $G_{a}\left(\alpha^{\prime}\right)$. Then:

$$
0 \neq a_{\left(\alpha, \alpha^{\prime}\right)} a_{\left(e, \alpha^{\prime}\right)}=a_{\left(e, \alpha^{\prime}\right)} b_{\left(\theta\left(\alpha^{\prime}\right) \star \alpha, \alpha^{\prime}\right)} .
$$

Therefore, $\alpha \in G_{b}\left(\alpha^{\prime}\right)$ : we obtain that $G_{a}\left(\alpha^{\prime}\right) \subseteq G_{b}\left(\alpha^{\prime}\right)$.

Let $\beta \in G_{b}\left(\alpha^{\prime}\right)$. Then $G_{b}\left(\alpha^{\prime}\right) \neq \varnothing$ is a subgroup of $H$, and $e \in G_{b}\left(\alpha^{\prime}\right)$. Hence:

$$
0 \neq b_{\left(\theta\left(\alpha^{\prime}\right), \alpha^{\prime}\right)} b_{\left(\theta\left(\alpha^{\prime}\right) \star \beta, \alpha^{\prime}\right)}=b_{\left(\theta\left(\alpha^{\prime}\right), \alpha^{\prime}\right)} a_{\left(\theta\left(\alpha^{\prime}\right)^{-1} \star \theta\left(\alpha^{\prime}\right) \star \beta, \alpha^{\prime}\right.} .
$$

We obtain that $\beta \in G_{a}\left(\alpha^{\prime}\right)$. Finally, for any $\alpha^{\prime} \in K, G_{a}\left(\alpha^{\prime}\right)=G_{b}\left(\alpha^{\prime}\right)$. We denote this set by $G\left(\alpha^{\prime}\right)$.

If $G\left(\alpha^{\prime}\right) \neq \varnothing$, we obtain, for $\alpha=\beta=e$ :

$$
a_{\alpha^{\prime}} b_{\alpha^{\prime}}=a_{\alpha^{\prime}} a_{\alpha^{\prime}}
$$


Consequently, $b_{\alpha^{\prime}}=a_{\alpha^{\prime}}$. We denote by $\lambda_{\alpha^{\prime}}$ this scalar.

As $m \neq 0$, at least one of the $G\left(\alpha^{\prime}\right)$ is nonempty. We consider

$$
K^{\prime}=\left\{\alpha^{\prime} \in K, G\left(\alpha^{\prime}\right) \neq \varnothing\right\} .
$$

Let $\alpha^{\prime}, \beta^{\prime} \in K^{\prime}$. For any $\alpha \in G\left(\alpha^{\prime}\right)$, for $\beta=e$ :

$$
\lambda_{\alpha^{\prime}} a_{\left(\alpha^{-1}, \beta^{\prime}\right)}=\lambda_{\alpha^{\prime}} \lambda_{\beta^{\prime}} \neq 0 .
$$

Hence, $\alpha^{-1} \in G\left(\beta^{\prime}\right)$. As this is a subgroup, $\alpha \in G\left(\beta^{\prime}\right)$, and $G\left(\alpha^{\prime}\right) \subseteq G\left(\beta^{\prime}\right)$. By symmetry, $G\left(\alpha^{\prime}\right)=G\left(\beta^{\prime}\right)$. We denote by $G$ this subset. Then:

$$
m=\sum_{\alpha^{\prime} \in K^{\prime}} \lambda_{\alpha^{\prime}} \sum_{\alpha \in G} \prec_{\left(\alpha, \alpha^{\prime}\right)}+>_{\left(\theta\left(\alpha^{\prime}\right) \star \alpha, \alpha^{\prime}\right)}=\sum_{\alpha^{\prime} \in K} \lambda_{\alpha^{\prime}} \sum_{\alpha \in G} \prec_{\left(\alpha, \alpha^{\prime}\right)}+>_{\left(\theta\left(\alpha^{\prime}\right) \star \alpha, \alpha^{\prime}\right)},
$$

where we put $\lambda_{\alpha^{\prime}}=0$ if $\alpha^{\prime} \notin K$. Conversely, for such a $\left.m, 45\right)$ is satisfied, so $m$ is associative.

Example 9. 1. If $H$ is a null group, we obtain the case of $\mathbf{E D S}(\Omega)$. The associative products are of the form

$$
\sum_{\alpha \in \Omega} \lambda_{\alpha}\left(\prec_{\alpha}+>_{\alpha}\right)
$$

where $\left(\lambda_{\alpha}\right)_{\alpha \in \Omega}$ is family of scalars with finite support.

2. If $K$ is reduced to a single element, we obtain the case of $\operatorname{EDS}^{*}(\Omega, \star, \omega)$. The associative products are of the form

$$
\lambda \sum_{\alpha \in H} \prec_{\alpha}+>_{\omega \star \alpha}
$$

where $\lambda$ is a scalar and $H$ is a subgroup of $(\Omega, \star)$.

Proposition 24. Let $\Omega$ be an EDS. We suppose that there exist $\alpha_{0}, \beta_{0} \in \Omega$ such that:

$$
\forall \alpha, \beta \in \Omega, \quad \alpha \triangleleft \beta=\beta_{0}, \quad \alpha \triangleright \beta=\alpha_{0} .
$$

The associative products of $\mathcal{P}_{\Omega}$ are of the form

$$
\lambda\left(<_{\beta_{0}}+>_{\alpha_{0}}\right),
$$

where $\lambda$ is a scalar.

Proof. Let $m$ be an associative product. In this case, 44 becomes:

$$
\begin{aligned}
& b_{\alpha} b_{\beta}= \begin{cases}\sum_{\alpha^{\prime} \rightarrow \beta^{\prime}=\alpha} b_{\alpha^{\prime}} b_{\beta^{\prime}} \text { if } \beta=\alpha_{0}, \\
0 \text { otherwise; }\end{cases} a_{\alpha} a_{\beta}=\left\{\begin{array}{l}
\sum_{\alpha^{\prime} \leftarrow \beta^{\prime}=\alpha} a_{\alpha^{\prime}} a_{\beta^{\prime}} \text { if } \beta=\beta_{0}, \\
0 \text { otherwise; }
\end{array}\right. \\
& b_{\alpha} a_{\beta}=\left\{\begin{array}{l}
\sum_{\alpha^{\prime} \leftarrow \beta^{\prime}=\alpha} b_{\alpha^{\prime}} b_{\beta^{\prime}} \text { if } \beta=\beta_{0}, \\
0 \text { otherwise; }
\end{array} a_{\alpha} b_{\beta}=\left\{\begin{array}{l}
\sum_{\alpha^{\prime} \rightarrow \beta^{\prime}=\alpha} a_{\alpha^{\prime}} a_{\beta^{\prime}} \text { if } \beta=\alpha_{0}, \\
0 \text { otherwise. }
\end{array}\right.\right.
\end{aligned}
$$

In particular, if $\alpha=\beta \neq \alpha_{0}, b_{\alpha}^{2}=0$, so $b_{\alpha}=0$. Similarly, if $\beta \neq \beta_{0}, a_{\beta}=0$. By (6), (8), 10) and (12):

$$
\alpha_{0} \leftarrow \alpha_{0}=\alpha_{0} \rightarrow \alpha_{0}=\alpha_{0}, \quad \beta_{0} \leftarrow \beta_{0}=\beta_{0} \rightarrow \beta_{0}=\beta_{0} .
$$

Hence, $b_{\alpha_{0}} a_{\beta_{0}}=b_{\alpha_{0}}^{2}$, so $b_{\alpha_{0}}=0$ or $b_{\alpha_{0}}=a_{\beta_{0}} ; a_{\beta_{0}} b_{\alpha_{0}}=a_{\beta_{0}}^{2}$, so $a_{\beta_{0}}=0$ or $b_{\alpha_{0}}=a_{\beta_{0}}$. Finally, $a_{\beta_{0}}=b_{\alpha_{0}}=\lambda$ and $m=\lambda\left(<_{\beta_{0}}+>_{\alpha_{0}}\right)$. The converse is trivial. 
Example 10. Let us give the associative products in the 24 four cases of cardinality 2 . Here, $\lambda$, $\mu$ and $\nu$ are scalars.

\begin{tabular}{|c|c|c|c|}
\hline$A 1$ & $\lambda\left(<_{a}+>_{a}\right)$ & $A 2$ & $\lambda\left(\prec_{a}+>_{a}\right)$ \\
\hline$B 1$ & $\lambda\left(\prec_{a}+>_{a}\right)$ & $B 2$ & $\lambda\left(\prec_{a}+>_{a}\right), \mu\left(>_{a}->_{b}\right)$ \\
\hline$C 1$ & $\lambda\left(\prec_{a}+>_{a}\right)$ & $C 2$ & $\lambda\left(\prec_{a}+>_{b}\right)$ \\
\hline$C 3$ & $\lambda\left(\prec_{a}+>_{a}\right), \mu\left(\prec_{b}+>_{b}\right)$ & $C 4$ & $\lambda\left(<_{b}+>_{a}\right)$ \\
\hline$C 5$ & $\lambda\left(<_{b}+>_{b}\right)$ & $D 1$ & $\lambda\left(<_{a}+>_{a}\right)$ \\
\hline$D 2$ & $\lambda\left(\prec_{a}+>_{a}\right), \mu\left(\prec_{a}-<_{b}\right)$ & $E 1$ & $\lambda\left(\prec_{a}+>_{a}\right)$ \\
\hline$E 2$ & $\lambda\left(\prec_{a}+>_{b}\right)$ & $E 3$ & $\lambda\left(<_{a}+>_{a}\right), \mu\left(<_{b}+>_{b}\right), \nu\left(<_{a}-<_{b}\right)$ \\
\hline$F 1$ & $\lambda\left(<_{a}+>_{a}\right)$ & $F 2$ & $\lambda\left(\prec_{a}+>_{b}\right)$ \\
\hline$F 3$ & $\lambda\left(<_{a}+>_{a}\right)+\mu\left(<_{b}+>_{b}\right)$ & $F 4$ & $\lambda\left(<_{a}+>_{a}\right), \mu\left(<_{a}+<_{b}+>_{a}+>_{b}\right)$ \\
\hline$F 5$ & $\lambda\left(\prec_{a}+>_{b}\right), \nu\left(\prec_{a}+<_{b}+>_{a}+>_{b}\right)$ & G1 & $\lambda\left(\prec_{a}+>_{a}\right)$ \\
\hline$G 2$ & $\lambda\left(<_{a}+>_{b}\right)$ & G3 & $\lambda\left(<_{a}+>_{a}\right), \mu\left(<_{b}+>_{b}\right), \nu\left(>_{a}->_{b}\right)$ \\
\hline$H 1$ & $\lambda\left(\prec_{a}+>_{a}\right)$ & $H 2$ & $\lambda\left(<_{a}+>_{a}\right), \mu\left(<_{a}+<_{b}+>_{a}+>_{b}\right)$ \\
\hline
\end{tabular}

\subsection{Dendriform products}

Proposition 25. Let $<,>\in \mathcal{P}(2)$, written under the form

$$
\prec=\sum_{\alpha \in \Omega} a_{\alpha} \prec_{\alpha}+\sum_{\alpha \in \Omega} b_{\alpha}>_{\alpha}, \quad>=\sum_{\alpha \in \Omega} c_{\alpha} \prec_{\alpha}+\sum_{\alpha \in \Omega} d_{\alpha}>_{\alpha},
$$

Then $(<,>)$ satisfies the dendriform relations

$$
<\circ(<, I)=<\circ(I,<+>), \quad<\circ(>, I)=>\circ(I,<), \quad>\circ(I,>)=>\circ(<+>, I)
$$

if, and only if, for any $\alpha, \beta \in \Omega$ :

$$
\begin{aligned}
& b_{\alpha} b_{\beta}=\sum_{\varphi \rightarrow\left(\alpha^{\prime}, \beta^{\prime}\right)=(\alpha, \beta)} b_{\alpha^{\prime}}\left(b_{\beta^{\prime}}+d_{\beta^{\prime}}\right), \quad a_{\alpha}\left(a_{\beta}+c_{\beta}\right)=\sum_{\varphi \leftarrow\left(\alpha^{\prime}, \beta^{\prime}\right)=(\alpha, \beta)} a_{\alpha^{\prime}} a_{\beta^{\prime}}, \\
& b_{\alpha} a_{\beta}=\sum_{\varphi \leftarrow\left(\alpha^{\prime}, \beta^{\prime}\right)=(\alpha, \beta)} b_{\alpha^{\prime}}\left(b_{\beta^{\prime}}+d_{\beta^{\prime}}\right), \quad a_{\alpha}\left(b_{\beta}+d_{\beta}\right)=\sum_{\varphi \rightarrow\left(\alpha^{\prime}, \beta^{\prime}\right)=(\alpha, \beta)} a_{\alpha^{\prime}} a_{\beta^{\prime}}, \\
& b_{\alpha} c_{\beta}=0, \\
& 0=\sum_{\varphi \leftarrow\left(\alpha^{\prime}, \beta^{\prime}\right)=(\alpha, \beta)} d_{\alpha^{\prime}} b_{\beta^{\prime}} \\
& 0=\sum_{\varphi \rightarrow\left(\alpha^{\prime}, \beta^{\prime}\right)=(\alpha, \beta)} c_{\alpha^{\prime}} a_{\beta^{\prime}} \\
& b_{\alpha} d_{\beta}=\sum_{\varphi \rightarrow\left(\alpha^{\prime}, \beta^{\prime}\right)=(\alpha, \beta)} d_{\alpha^{\prime}} b_{\beta^{\prime}}, \\
& c_{\alpha} a_{\beta}=\sum_{\varphi \leftarrow\left(\alpha^{\prime}, \beta^{\prime}\right)=(\alpha, \beta)} c_{\alpha^{\prime}} a_{\beta^{\prime}}, \\
& c_{\alpha} c_{\beta}=\sum_{\varphi \leftarrow\left(\alpha^{\prime}, \beta^{\prime}\right)=(\alpha, \beta)}\left(a_{\alpha^{\prime}}+c_{\alpha^{\prime}}\right) c_{\beta^{\prime}}, \\
& d_{\alpha}\left(b_{\beta}+d_{\beta}\right)=\sum_{\varphi \rightarrow\left(\alpha^{\prime}, \beta^{\prime}\right)=(\alpha, \beta)} d_{\alpha^{\prime}} d_{\beta^{\prime}}, \\
& c_{\alpha} d_{\beta}=\sum_{\varphi \rightarrow\left(\alpha^{\prime}, \beta^{\prime}\right)=(\alpha, \beta)}\left(a_{\alpha^{\prime}}+c_{\alpha^{\prime}}\right) c_{\beta^{\prime}}, \\
& d_{\alpha}\left(a_{\beta}+c_{\beta}\right)=\sum_{\varphi \leftarrow\left(\alpha^{\prime}, \beta^{\prime}\right)=(\alpha, \beta)} d_{\alpha^{\prime}} d_{\beta^{\prime}} .
\end{aligned}
$$

Proof. By direct computations in the operad $\mathcal{P}_{\Omega}$, as for Proposition 21.

Note that if $(<,>)$ satisfies the dendriform relations, then $\amalg=<+>$ is associative:

$$
\uplus \circ(\amalg, I)=\uplus \circ(I, \uplus) .
$$

In the nondegenerate case, the knowledge of the associative products of $\mathcal{P} \Omega$ induces the knowledge of all dendriform products: 
Corollary 26. Let $\Omega$ be a nondegenerate EDS. For any associative product

$$
m=\sum_{\alpha \in \Omega} a_{\alpha} \prec_{\alpha}+\sum_{\alpha \in \Omega} d_{\alpha}>_{\alpha} \in \mathcal{P}(2),
$$

the only pairs of dendriform products $(<,>)$ such that $\langle+>=m$ are the following:

$$
(m, 0), \quad(0, m), \quad\left(\sum_{\alpha \in \Omega} a_{\alpha} \prec_{\alpha}, \sum_{\alpha \in \Omega} d_{\alpha}>_{\alpha}\right) .
$$

Proof. Let $(<,>)$ be a pair of dendriform products and $m=<+>$. As $\varphi_{\leftarrow}$ and $\varphi_{\rightarrow}$ are bijective, 466 gives (third, fourth fifth and eighth rows, first column) that:

$$
\forall \alpha, \beta \in \Omega, \quad b_{\alpha} c_{\beta}=0, \quad d_{\alpha} b_{\beta}=0, \quad c_{\alpha} a_{\beta}=0, \quad c_{\alpha} b_{\beta}=0 .
$$

If one of the $b_{\beta}$ is nonzero, then for any $\alpha \in \Omega, c_{\alpha}=d_{\alpha}=0$, so $>=0$ and $\prec=m$. Similarly, if one of the $c_{\beta}$ is nonzero, then for $\prec=0$ and $>=m$. If for any $\beta \in \Omega, b_{\beta}=c_{\beta}=0$, then:

$$
\prec=\sum_{\alpha \in \Omega} a_{\alpha} \prec_{\alpha}, \quad>=\sum_{\alpha \in \Omega} d_{\alpha}>_{\alpha} .
$$

Conversely, if $m$ is an associative product, written under the form:

$$
m=\sum_{\alpha \in \Omega} a_{\alpha} \prec_{\alpha}+d_{\alpha}>_{\alpha}
$$

then obviously, $(0, m)$ and $(m, 0)$ are pairs of dendriform products. If we define $(<,>)$ by (47), that is to say $b_{\alpha}=c_{\alpha}=0$ for any $\alpha \in \Omega$, then (44) implies (46), so $(<,>)$ is dendriform.

\subsection{Koszul dual}

When $\Omega$ is finite, the operad $\mathcal{P}_{\Omega}$ is a quadratic algebra, finitely generated. By direct computations, we obtain the Koszul dual of $\mathcal{P}_{\Omega}$ :

Proposition 27. Let $\Omega$ be a finite EDS. The Koszul dual $\mathcal{P}_{\Omega}^{!}$of $\mathcal{P}_{\Omega}$ is generated by the elements $\dashv_{\alpha}, \vdash_{\alpha}, \alpha \in \Omega$, with the relations:

$$
\begin{aligned}
\forall \alpha, \beta \in \Omega, & \dashv_{\beta} \circ\left(\dashv_{\alpha}, I\right)=\dashv_{\alpha \leftarrow \beta} \circ\left(I, \dashv_{\alpha \triangleleft \beta}\right)=\dashv_{\alpha \rightarrow \beta} \circ\left(I, \vdash_{\alpha \triangleright \beta}\right), \\
& \vdash_{\beta} \circ\left(I, \dashv_{\alpha}\right)=\dashv_{\alpha} \circ\left(\vdash_{\beta}, I\right), \\
& \vdash_{\alpha} \circ\left(I \vdash_{\beta}\right)=\vdash_{\alpha \rightarrow \beta} \circ\left(\vdash_{\alpha \triangleright \beta}, I\right)=\vdash_{\alpha \leftarrow \beta} \circ\left(\dashv_{\alpha \triangleleft \beta}, I\right) .
\end{aligned}
$$

Definition 28. Let $(\Omega, \leftarrow, \rightarrow, \triangleleft, \triangleright)$ be an EDS. We consider the following linear map:

$$
\varphi:\left\{\begin{array}{rll}
\mathbb{K} \Omega^{2} & \longrightarrow & \mathbb{K} \Omega^{2} \times \mathbb{K} \Omega^{2} \\
(\alpha, \beta) & \longrightarrow & \left(\varphi_{\leftarrow}(\alpha, \beta), \varphi_{\rightarrow}(\alpha, \beta)\right) .
\end{array}\right.
$$

The dimension of the kernel of $\varphi$ is called the corank of $\Omega$ and denoted by $\operatorname{coRk}(\Omega)$. We shall say that $\Omega$ is weakly nondegenerate if $\operatorname{coRk}(\Omega)=0$.

Example 11. 1. If $\varphi_{\leftarrow}$ or $\varphi_{\rightarrow}$ is injective (which happens if $\Omega$ is nondegenerate), then $\Omega$ is weaky nondegenerate.

2. If $(\Omega, \leftarrow, \rightarrow)$ is a diassociative semigroup, then $\operatorname{EDS}(\Omega, \leftarrow, \rightarrow)$ is weakly nondegenerate. Indeed, in this case,

$$
\varphi(\alpha, \beta)=((\alpha \leftarrow \beta, \beta),(\alpha, \rightarrow \beta, \beta)),
$$

so $\varphi$ is injective. 
3. Here are the coranks of the 24 EDS of cardinality 2.

\begin{tabular}{|c|c||c|c||c|c||c|c|}
\hline$A 1$ & 3 & $A 2$ & 1 & $B 1$ & 2 & $B 2$ & 0 \\
\hline$C 1$ & 2 & $C 2$ & 2 & $C 3$ & 0 & $C 4$ & 2 \\
\hline$C 5$ & 2 & $D 1$ & 2 & $D 2$ & 0 & $E 1$ & 2 \\
\hline$E 2$ & 2 & $E 3$ & 0 & $F 1$ & 1 & $F 2$ & 1 \\
\hline$F 3$ & 0 & $F 4$ & 0 & $F 5$ & 0 & $G 1$ & 2 \\
\hline$G 2$ & 2 & $G 3$ & 0 & $H 1$ & 2 & $H 2$ & 0 \\
\hline
\end{tabular}

Proposition 29. Let $(\Omega, \leftarrow, \rightarrow, \triangleleft, \triangleright)$. Then:

$$
\operatorname{dim}_{\mathbb{K}}\left(\mathcal{P}_{\Omega}^{!}(3)\right)=3|\Omega|^{2}+2 \operatorname{coRk}(\Omega) .
$$

Proof. For any $m^{(1)}, m^{(2)} \in\{\dashv, \vdash\}$, we shall consider the following subspaces of the free operad generated by $\vdash_{\alpha}, \dashv_{\alpha}$, with $\alpha \in \Omega$ :

$$
\begin{aligned}
& L\left(m_{1}, m_{2}\right)=\operatorname{Vect}\left(m_{\alpha}^{(1)} \circ\left(m_{\beta}^{(2)}, I\right), \alpha, \beta \in \Omega\right), \\
& R\left(m_{1}, m_{2}\right)=\operatorname{Vect}\left(m_{\alpha}^{(1)} \circ\left(I, m_{\beta}^{(2)}\right), \alpha, \beta \in \Omega\right) .
\end{aligned}
$$

According to the form of the relations defining $\mathcal{P}_{\Omega}^{!}$:

$$
\mathcal{P}_{\Omega}^{!}(3)=R(\vdash, \dashv) \oplus \frac{L(\vdash, \vdash) \oplus L(\vdash, \dashv)}{E_{1}} \oplus \frac{R(\dashv, \dashv) \oplus R(\dashv, \vdash)}{E_{2}},
$$

with:

$$
\begin{aligned}
& E_{1}=V e c t\left(\vdash_{\alpha \leftarrow \beta} \circ\left(\dashv_{\alpha \triangleleft \beta}, I\right)-\vdash_{\alpha \rightarrow \beta} \circ\left(\vdash_{\alpha \triangleright \beta}, I\right),(\alpha, \beta) \in \Omega^{2}\right), \\
& E_{2}=\operatorname{Vect}\left(\dashv_{\alpha \leftarrow \beta} \circ\left(I, \dashv_{\alpha \triangleleft \beta}\right)-\dashv_{\alpha \rightarrow \beta} \circ\left(I, \vdash_{\alpha \triangleright \beta}\right),(\alpha, \beta) \in \Omega^{2}\right) .
\end{aligned}
$$

Hence:

$$
\operatorname{dim}_{\mathbb{K}}\left(\mathcal{P}_{\Omega}^{!}(3)\right)=5|\Omega|^{2}-\operatorname{dim}_{\mathbb{K}}(E 1)-\operatorname{dim}_{\mathbb{K}}\left(E_{2}\right) .
$$

By definition of $\varphi, \operatorname{dim}_{\mathbb{K}}\left(E_{1}\right)=\operatorname{dim}_{\mathbb{K}}\left(E_{2}\right)=\operatorname{dim}_{\mathbb{K}}(\operatorname{Im}(\varphi))=|\Omega|^{2}-\operatorname{coRk}(\Omega)$, which gives the result.

Theorem 30. Let $\Omega$ be an extended diassociative dialgebra.

1. If $\mathcal{P}_{\Omega}$ is Koszul, then $\Omega$ is weaky nondegenerate.

2. If $\Omega$ is nondegenerate, then $\mathcal{P}_{\Omega}$ is Koszul.

Proof. We put $\omega=|\Omega|$.

1. Let us assume that $\mathcal{P}_{\Omega}$ is Koszul. The Poincaré-Hilbert formal series of $\mathcal{P}_{\Omega}$ is:

$$
F=\sum_{k=1}^{\infty} \operatorname{dim}_{\mathbb{K}}\left(\mathcal{P}_{\Omega}(n)\right) X^{n}=X+2 \omega X^{2}+5 \omega^{2} X^{3}+\ldots=\frac{1-\sqrt{1-4 \omega X}}{2 \omega^{2} X}-\frac{1}{\omega} .
$$

We denote by $G$ the Poincaré-Hilbert formal series of $\mathcal{P}_{\Omega}^{!}$. As $\mathcal{P}_{\Omega}$ is Koszul:

$$
F(-G(-X))=X
$$

so:

$$
G=\frac{X}{(1-\omega X)^{2}}=\sum_{n=1}^{\infty} n \omega^{n-1} X^{n},
$$

so $\operatorname{dim}_{\mathbb{K}}\left(\mathcal{P}_{\Omega}^{!}(3)\right)=3 \omega^{2}$. Therefore, $\operatorname{coRk}(\Omega)=0$. 
2. We use the rewriting method of [2] to prove that $\mathcal{P}_{\Omega}^{!}$is Koszul. The rewriting rules are the following:
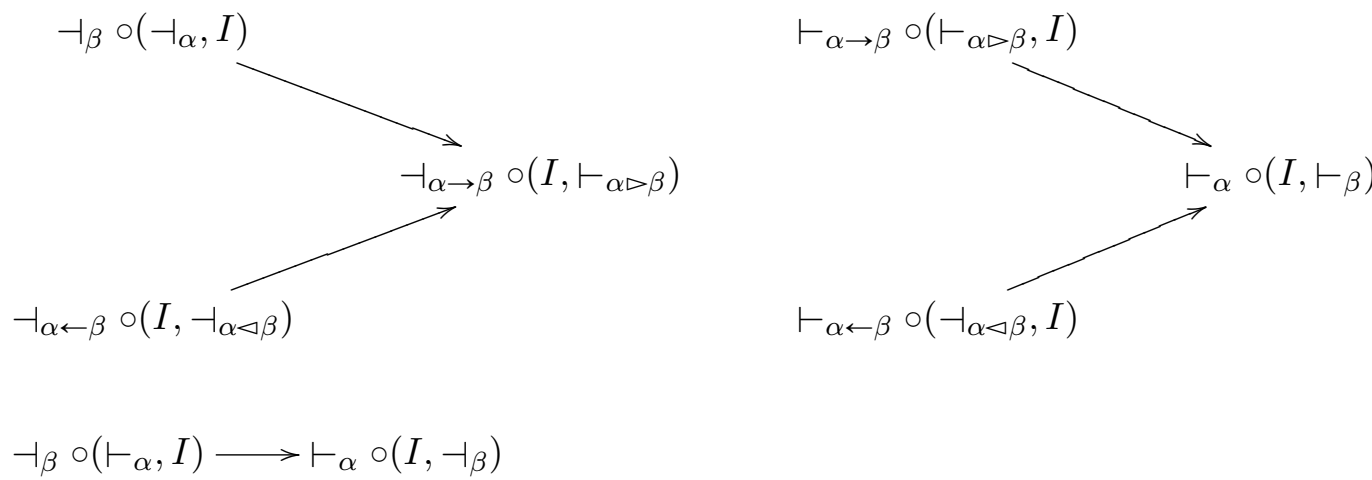

There are 14 critical trees, giving 14 diagrams which turn out to be all confluent. Let us describe two of them.

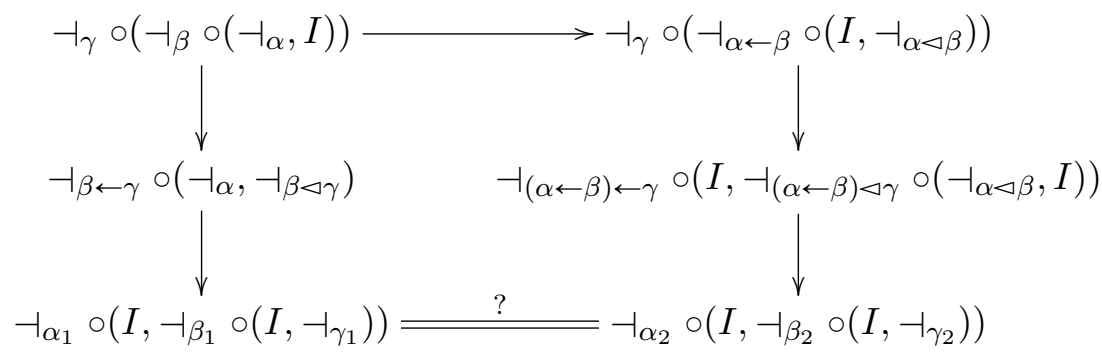

with:

$$
\begin{array}{ll}
\alpha_{1}=\alpha \leftarrow(\beta \leftarrow \gamma), & \alpha_{2}=(\alpha \leftarrow \beta) \leftarrow \gamma, \\
\beta_{1}=\alpha \triangleleft(\beta \leftarrow \gamma), & \beta_{2}=(\alpha \triangleleft \beta) \leftarrow((\alpha \leftarrow \beta) \triangleleft \gamma), \\
\gamma_{1}=\beta \triangleleft \gamma, & \gamma_{2}=(\alpha \triangleleft \beta) \triangleleft((\alpha \leftarrow \beta) \triangleleft \gamma) .
\end{array}
$$

By (1), (6) and (7), $\left(\alpha_{1}, \beta_{1}, \gamma_{1}\right)=\left(\alpha_{2}, \beta_{2}, \gamma_{2}\right)$.

We denote the inverse of $\varphi_{\leftarrow}$ by $\psi_{\leftarrow}=\left(\psi_{\leftarrow}^{1}, \psi_{\leftarrow}^{2}\right)$.

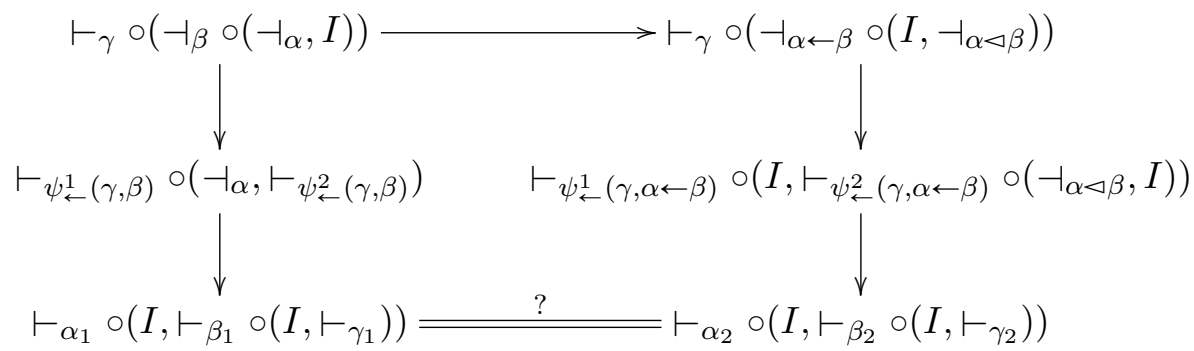

with:

$$
\begin{aligned}
\alpha_{1} & =\psi_{\leftarrow}^{1}\left(\psi_{\leftarrow}^{1}(\gamma, \beta), \alpha\right), & & \alpha_{2}=\psi_{\leftarrow}^{1}(\gamma, \alpha \leftarrow \beta), \\
\beta_{1} & =\psi_{\leftarrow}^{1}\left(\psi_{\leftarrow}^{1}(\gamma, \beta), \alpha\right), & & \beta_{2}=\psi_{\leftarrow}^{1}\left(\psi_{\leftarrow}^{2}(\gamma, \alpha \leftarrow \beta), \alpha \triangleleft \beta\right), \\
\gamma_{1} & =\psi_{\leftarrow}^{2}(\gamma, \beta), & & \gamma_{2}=\psi_{\leftarrow}^{1}\left(\psi_{\leftarrow}^{2}(\gamma, \alpha \leftarrow \beta), \alpha \triangleleft \beta\right) .
\end{aligned}
$$

By definition:

$$
(\varphi \leftarrow \otimes I d) \circ\left(I d \otimes \varphi_{\leftarrow}\right)\left(\alpha_{2}, \beta_{2}, \gamma_{2}\right)=(\gamma, \alpha \leftarrow \beta, \alpha \triangleleft \beta) .
$$

Let us compute

$$
\left(\varphi_{\leftarrow} \otimes I d\right) \circ\left(I d \otimes \varphi_{\leftarrow}\right)\left(\alpha_{1}, \beta_{1}, \gamma_{1}\right)=\left(\alpha_{1}^{\prime}, \beta_{1}^{\prime}, \gamma_{1}^{\prime}\right) .
$$


We obtain, by (1):

$$
\alpha_{1}^{\prime}=\alpha_{1} \leftarrow\left(\beta \leftarrow \gamma_{1}\right)=\left(\alpha_{1} \leftarrow \beta_{1}\right) \leftarrow \gamma_{1}=\psi_{1}(\gamma, \beta) \leftarrow \psi_{2}(\gamma, \beta)=\gamma .
$$

Moreover, by (6):

$$
\beta_{1}^{\prime}=\alpha_{1} \triangleleft\left(\beta_{1} \leftarrow \gamma_{1}\right)=\left(\alpha_{1} \triangleleft \beta_{1}\right) \leftarrow\left(\left(\alpha_{1} \leftarrow \beta_{1}\right) \triangleleft \gamma_{1}\right)=\alpha \leftarrow\left(\psi_{\leftarrow}^{1}(\gamma, \beta) \triangleleft \psi_{\leftarrow}^{2}(\gamma, \beta)\right)=\alpha \leftarrow \beta .
$$

By (7):

$$
\gamma_{1}^{\prime}=\beta_{1} \triangleleft \gamma_{1}=\left(\alpha_{1} \triangleleft \beta_{1}\right) \triangleleft\left(\left(\alpha_{1} \leftarrow \beta_{1}\right) \triangleleft \gamma_{1}=\alpha \triangleleft\left(\psi_{\leftarrow}^{1}(\gamma, \beta) \triangleleft \psi_{\leftarrow}^{2}(\gamma, \beta)\right)=\alpha \triangleleft \beta .\right.
$$

So:

$$
\left(\varphi_{\leftarrow} \otimes I d\right) \circ\left(I d \otimes \varphi_{\leftarrow}\right)\left(\alpha_{1}, \beta_{1}, \gamma_{1}\right)=\left(\varphi_{\leftarrow} \otimes I d\right) \circ\left(I d \otimes \varphi_{\leftarrow}\right)\left(\alpha_{2}, \beta_{2}, \gamma_{2}\right) .
$$

As $\varphi_{\leftarrow}$ is injective, $\left(\alpha_{1}, \beta_{1}, \gamma_{1}\right)=\left(\alpha_{2}, \beta_{2}, \gamma_{2}\right)$.

Example 12. The first point implies that the operads associated to the EDS A1, A2, B1, C1, C2, C4, C5, D1, E1, E2, G1, G2 and H1 are not Koszul. The second point implies that the operads associated to the EDS F2, F3, F4, F5, H2 are Koszul. We do not know if the operads associated to B2, C3, D2, E3 and G3 are Koszul or not.

\section{Combinatorial description of the products}

\subsection{On typed trees}

Definition 31. Let $k \geqslant 0, \alpha_{2}, \ldots, \alpha_{k} \in \Omega, \beta_{1}, \ldots, \beta_{k} \in \Omega \sqcup\{\varnothing\}$ and $T_{1}, \ldots, T_{k} \in \Omega$, with the convention that $\beta_{i}=\varnothing$ if and only if $T_{i}=1$. We put:

$$
\begin{aligned}
& R_{\left(\alpha_{2}, \ldots, \alpha_{k}\right)}\left(\beta_{1} \otimes T_{1}, \ldots, \beta_{k} \otimes T_{k}\right)=\left\{\begin{array}{l}
\text { I if } k=0, \\
T_{1} \bigvee_{\alpha_{1}, \alpha_{2}} R_{\left(\alpha_{3}, \ldots, \alpha_{k}\right)}\left(\beta_{2} \otimes T_{2}, \ldots, \beta_{k} \otimes T_{k}\right) \text { if } k \geqslant 2 ;
\end{array}\right. \\
& L_{\left(\alpha_{2}, \ldots, \alpha_{k}\right)}\left(\beta_{1} \otimes T_{1}, \ldots, \beta_{k} \otimes T_{k}\right)=\left\{\begin{array}{l}
\text { I if } k=0, \\
L_{\left(\alpha_{3}, \ldots, \alpha_{k}\right)}\left(\beta_{2} \otimes T_{2}, \ldots, \beta_{k} \otimes T_{k}\right) \bigvee_{\alpha_{2}, \alpha_{1}} T_{1} \text { if } \geqslant 2 .
\end{array}\right.
\end{aligned}
$$

Let us denote by $L_{k}$ the ladder of length $k$ :

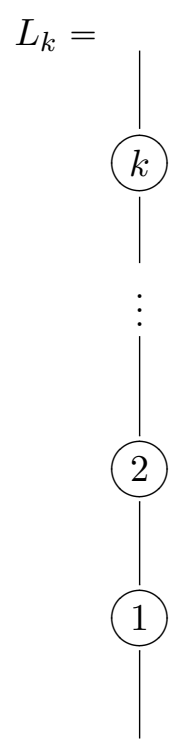


Roughly speaking, $R_{\left(\alpha_{2}, \ldots, \alpha_{k}\right)}\left(\beta_{1} \otimes T_{1}, \ldots, \beta_{k} \otimes T_{k}\right)$, (respectively $\left.L_{\left(\alpha_{2}, \ldots, \alpha_{k}\right)}\left(\beta_{1} \otimes T_{1}, \ldots, \beta_{k} \otimes T_{k}\right)\right)$ is obtained by grafting $T_{i}$ on the vertex $i$ of $L_{k}$ for any $i$ on the left (respectively on the right). The type of the edge from $i$ to the root of $T_{i}$ is $\beta_{i}$; the type of the root between the vertex $i-1$ and the vertex $i$ is $\alpha_{i}$ for any $i \geqslant 2$.

Note that any tree $T$ in $\mathcal{T}_{\Omega}$ can uniquely written under the form

$$
T=R_{\left(\alpha_{2}, \ldots, \alpha_{k}\right)}\left(\beta_{1} \otimes T_{1}, \ldots, \beta_{k} \otimes T_{k}\right) .
$$

This is the right comb decomposition of $T$. It can also be uniquely written under the form

$$
T=L_{\left(\alpha_{2}^{\prime}, \ldots, \alpha_{l}^{\prime}\right)}\left(\beta_{1}^{\prime} \otimes T_{1}^{\prime}, \ldots, \beta_{l}^{\prime} \otimes T_{l}^{\prime}\right) .
$$

This is the left comb decomposition of $T$.

Definition 32. Let $k, l \geqslant 0$. A $(k, l)$-shuffle is a permutation $\sigma \in \mathfrak{S}_{k+l}$ such that

$$
\sigma(1)<\ldots<\sigma(k), \quad \sigma(k+1)<\ldots<\sigma(k+l) .
$$

The set of $(k, l)$-shuffle will be denoted by $\operatorname{sh}(k, l)$. If $\sigma \in \mathbf{s h}(k, l)$, then $\sigma^{-1}(1) \in\{1, k+1\}$ : we put

$$
\operatorname{sh}_{<}(k, l)=\left\{\sigma \in \operatorname{sh}(k, l), \sigma^{-1}(1)=1\right\}, \quad \operatorname{sh}_{>}(k, l)=\left\{\sigma \in \operatorname{sh}(k, l), \sigma^{-1}(1)=k+1\right\} .
$$

Notations 2. Let $k, l \geqslant 0, \sigma \in \operatorname{sh}(k, l), \alpha_{2}, \ldots, \alpha_{k+l} \in \Omega, \beta_{1}, \ldots, \beta_{k+l} \in \Omega \sqcup\{\varnothing\}$ and $T_{1}, \ldots, T_{k+l} \in$ $\Omega$, with the convention that $\beta_{i}=\varnothing$ if and only if $T_{i}=$ ।. Let

$$
T_{\sigma}^{\left(\alpha_{2}, \ldots, \alpha_{k+l}\right)}\left(\beta_{1} \otimes T_{1}, \ldots, \beta_{k} \otimes T_{k} ; \beta_{k+1} \otimes T_{k+1}, \ldots, \beta_{k+l} \otimes T_{k+l}\right)
$$

be the typed tree obtained in the following process: starting form the ladder $L_{k+l}$,

- For any $1 \leqslant i \leqslant k+l$, graft $T_{\sigma^{-1}(i)}$ on the vertex $i$, on the left if $\sigma^{-1}(i) \leqslant k$, and on right otherwise.

- The type of the edge between the vertex $i$ and the root of $T_{\sigma^{-1}(i)}$ is $\beta_{\sigma^{-1}(i)}$.

- The type of the edge between the vertex $i-1$ and $i$ is $\alpha_{i}$ for any $i \geqslant 2$.

Notations 3. Let $k, l \geqslant 0$, with $k+l \geqslant 1$, and $\sigma \in \operatorname{sh}(k, l)$. We define a map $D_{\sigma}^{k, l}: \Omega^{k+l-1} \longrightarrow$ $\Omega^{k+l-1}$ :

- If $k=0$ or $l=0$, then $\sigma=I d_{k+l}$.We put $D_{\sigma}^{k, l}=I d_{\Omega^{k+l-1}}$.

- Otherwise:

- If $\sigma \in \mathbf{s h}_{<}(1, l)$, then $\sigma=I d_{l+1}$, and

$$
D_{\sigma}^{1, l}\left(\alpha_{2}, \ldots, \alpha_{k+1}\right)=\left(\alpha_{2}, \ldots, \alpha_{k+1}\right) .
$$

- If $\sigma \in \mathbf{s h}_{<}(k, l)$, with $k \geqslant 2$, let $\sigma^{\prime}$ be the following permutation:

$$
\sigma^{\prime}=(\sigma(2)-1, \ldots, \sigma(k+l)-1) \in \mathbf{s h}(k-1, l) .
$$

If $\sigma^{\prime} \in \mathbf{s h}_{<}(k-1, l)$, for any $\left(\alpha_{2}, \ldots, \alpha_{k+l}\right) \in \Omega^{k+l-1}$ :

$$
D_{\sigma}^{k, l}\left(\alpha_{2}, \ldots, \alpha_{k+l}\right)=\left(\alpha_{2} \leftarrow \alpha_{k+1}, D_{\sigma^{\prime}}^{k-1, l}\left(\alpha_{3}, \ldots, \alpha_{k}, \alpha_{2} \triangleleft \alpha_{k+1}, \ldots, \alpha_{k+l}\right)\right) .
$$

If $\sigma^{\prime} \in \mathbf{s h}_{>}(k-1, l)$, for any $\left(\alpha_{2}, \ldots, \alpha_{k+l}\right) \in \Omega^{k+l-1}$ :

$$
D_{\sigma}^{k, l}\left(\alpha_{2}, \ldots, \alpha_{k+l}\right)=\left(\alpha_{2} \rightarrow \alpha_{k+1}, D_{\sigma^{\prime}}^{k-1, l}\left(\alpha_{3}, \ldots, \alpha_{k}, \alpha_{2} \triangleright \alpha_{k+1}, \ldots, \alpha_{k+l}\right)\right) .
$$


- If $\sigma \in \mathbf{s h}_{>}(k, 1)$, then $\sigma=(2,3 \ldots, k+1,1)$ and

$$
D_{\sigma}^{k, 1}\left(\alpha_{2}, \ldots, \alpha_{k+1}\right)=\left(\alpha_{k+1}, \alpha_{2}, \ldots, \alpha_{k}\right)
$$

- If $\sigma \in \mathbf{s h}_{>}(k, l)$, with $l \geqslant 2$, let $\sigma^{\prime}$ be the following permutation:

$$
\sigma^{\prime}=(\sigma(1)-1, \ldots, \sigma(k)-1, \sigma(k+2)-1, \sigma(k+l)-1) \in \mathbf{s h}(k, l-1) .
$$

If $\sigma^{\prime} \in \mathbf{s h}_{<}(k, l-1)$, for any $\left(\alpha_{2}, \ldots, \alpha_{k+l}\right) \in \Omega^{k+l-1}$ :

$$
D_{\sigma}^{k, l}\left(\alpha_{2}, \ldots, \alpha_{k+l}\right)=\left(\alpha_{k+1} \leftarrow \alpha_{k+2}, D_{\sigma^{\prime}}^{k-1, l}\left(\alpha_{2}, \ldots, \alpha_{k}, \alpha_{k+1} \triangleleft \alpha_{k+2}, \ldots, \alpha_{k+l}\right)\right)
$$

If $\sigma^{\prime} \in \mathbf{s h}_{\succ}(k, l-1)$, for any $\left(\alpha_{2}, \ldots, \alpha_{k+l}\right) \in \Omega^{k+l-1}$ :

$$
D_{\sigma}^{k, l}\left(\alpha_{2}, \ldots, \alpha_{k+l}\right)=\left(\alpha_{k+1} \rightarrow \alpha_{k+2}, D_{\sigma^{\prime}}^{k-1, l}\left(\alpha_{2}, \ldots, \alpha_{k}, \alpha_{k+1} \triangleright \alpha_{k+2}, \ldots, \alpha_{k+l}\right)\right)
$$

Example 13.

$$
\begin{array}{ll}
D_{(123)}^{2,1}\left(\alpha_{2}, \alpha_{3}\right)=\left(\alpha_{2} \leftarrow \alpha_{3}, \alpha_{2} \triangleleft \alpha_{3}\right), & D_{(213)}^{1,2}\left(\alpha_{2}, \alpha_{3}\right)=\left(\alpha_{2} \leftarrow \alpha_{3}, \alpha_{2} \triangleleft \alpha_{3}\right), \\
D_{(132)}^{2,1}\left(\alpha_{2}, \alpha_{3}\right)=\left(\alpha_{2} \rightarrow \alpha_{3}, \alpha_{2} \triangleright \alpha_{3}\right), & D_{(312)}^{1,2}\left(\alpha_{2}, \alpha_{3}\right)=\left(\alpha_{2} \rightarrow \alpha_{3}, \alpha_{2} \triangleright \alpha_{3}\right), \\
D_{(231)}^{2,1}\left(\alpha_{2}, \alpha_{3}\right)=\left(\alpha_{3}, \alpha_{2}\right), & D_{(123)}^{1,2}\left(\alpha_{2}, \alpha_{3}\right)=\left(\alpha_{2}, \alpha_{3}\right) .
\end{array}
$$

Proposition 33. Let us consider two elements of $\mathcal{T}_{\Omega}$ :

$$
\begin{aligned}
T & =R_{\left(\alpha_{2}, \ldots, \alpha_{k}\right)}\left(\beta_{1} \otimes T_{1}, \ldots, \beta_{k} \otimes T_{k}\right), \\
T^{\prime} & =L_{\left(\alpha_{k+2}, \ldots, \alpha_{k+l}\right)}\left(\beta_{k+1} \otimes T_{k+1}, \ldots, \beta_{k+l} \otimes T_{k+l}\right)
\end{aligned}
$$

Let $\alpha_{k+1} \in \Omega$. Then:

$$
\begin{aligned}
T \prec_{\alpha_{k+1}} T^{\prime} & =\sum_{\sigma \in \mathbf{s} \mathbf{h}_{<}(k, l)} T_{\sigma}^{D_{\sigma}^{k, l}\left(\alpha_{2}, \ldots, \alpha_{k+l}\right)}\left(\beta_{1} \otimes T_{1}, \ldots, \beta_{k} \otimes T_{k} ; \beta_{k+1} \otimes T_{k+1}, \ldots, \beta_{k+l} \otimes T_{k+l}\right), \\
T>_{\alpha_{k+1}} T^{\prime} & =\sum_{\sigma \in \mathbf{s} \mathbf{h}_{>}(k, l)} T_{\sigma}^{D_{\sigma}^{k, l}\left(\alpha_{2}, \ldots, \alpha_{k+l}\right)}\left(\beta_{1} \otimes T_{1}, \ldots, \beta_{k} \otimes T_{k} ; \beta_{k+1} \otimes T_{k+1}, \ldots, \beta_{k+l} \otimes T_{k+l}\right) .
\end{aligned}
$$

Proof. If $k=0$, observe that:

$$
\begin{aligned}
\mathbf{s h}_{<}(0, l) & =\varnothing, & \operatorname{sh}_{>}(0, l) & =\left\{I d_{l}\right\}, \\
\mid<<_{\alpha_{1}} T^{\prime} & =0, & \mid>_{\alpha_{1}} T^{\prime} & =T^{\prime} .
\end{aligned}
$$

So the result is immediate if $k=0$. It is proved in the same way if $l=0$. We now assume that $k, l \geqslant 1$, and we proceed by induction on $k+l$. There is nothing more to prove if $k+l \leqslant 1$. 
Otherwise, by the induction hypothesis, putting $S=R_{\left(\alpha_{3}, \ldots, \alpha_{k}\right)}\left(\beta_{2} \otimes T_{2}, \ldots, \beta_{k} \otimes T_{k}\right)$ :

$$
\begin{aligned}
T \prec_{\alpha_{k+1}} T^{\prime} & =T_{1} \bigvee_{\beta_{1}, \alpha_{2}} S \prec_{\alpha_{k+1}} T^{\prime} \\
& =T_{1} \bigvee_{\beta_{1}, \alpha_{2} \leftarrow \alpha_{k+1}}\left(S \prec_{\alpha_{2} \triangleleft \alpha_{k+1}} T^{\prime}\right)+T_{1} \bigvee_{\beta_{1}, \alpha_{2} \rightarrow \alpha_{k+1}}\left(S>_{\alpha_{2} \triangleright \alpha_{k+1}} T^{\prime}\right) \\
& =\sum_{\sigma \in \mathbf{s h}_{<}(k-1, l)} T_{1} \bigvee_{\beta_{1}, \alpha_{2} \leftarrow \alpha_{k+1}} T_{\sigma}^{D_{\sigma}^{k-1, l}\left(\alpha_{3}, \ldots, \alpha_{2} \triangleleft \alpha_{k+1}, \ldots \alpha_{k+l}\right)}\left(\beta_{2} \otimes T_{2}, \ldots, \beta_{k+l} \otimes T_{k+l}\right) \\
& +\sum_{\sigma \in \mathbf{s h}_{\succ}(k-1, l)} T_{1} \bigvee_{\beta_{1}, \alpha_{2} \rightarrow \alpha_{k+1}} T_{\sigma}^{D_{\sigma}^{k-1, l}\left(\alpha_{3}, \ldots, \alpha_{2} \triangleright \alpha_{k+1}, \ldots \alpha_{k+l}\right)}\left(\beta_{2} \otimes T_{2}, \ldots, \beta_{k+l} \otimes T_{k+l}\right) \\
& =\sum_{\sigma \in \mathbf{s h}_{<}(k-1, l)} T_{\bar{\sigma}}^{D_{\bar{\sigma}}^{k, l}\left(\alpha_{2}, \ldots, \alpha_{k+l}\right)}\left(\beta_{1} \otimes T_{1}, \ldots, \beta_{k+l} \otimes T_{k+l}\right) \\
& +\sum_{\sigma \in \mathbf{s h}_{\succ}(k-1, l)} T_{\bar{\sigma}}^{D_{\bar{\sigma}}^{k, l}\left(\alpha_{2}, \ldots, \alpha_{k+l}\right)}\left(\beta_{1} \otimes T_{1}, \ldots, \beta_{k+l} \otimes T_{k+l}\right) \\
& =\sum_{\sigma \in \operatorname{Sh}_{(}(k, l)} T_{\sigma}^{D_{\sigma}^{k, l}\left(\alpha_{2}, \ldots, \alpha_{k+l}\right)}\left(\beta_{1} \otimes T_{1}, \ldots, \beta_{k+l} \otimes T_{k+l}\right),
\end{aligned}
$$

where $\bar{\sigma}=(1, \sigma(1)+1, \ldots, \sigma(k+l-1)+1)$. The formula for $T>_{\alpha_{k+1}} T^{\prime}$ is proved in the same way.

The formulas for $D_{\sigma}^{k, l}$ can be simplified when $\triangleleft$ and $\triangleright$ are trivial:

Proposition 34. Let $(\Omega, \leftarrow, \rightarrow)$ be a diassociative semigroup. We work with the $\operatorname{EDS} \operatorname{EDS}(\Omega)$. Let $k, l \geqslant 0, \sigma \in \operatorname{sh}(k, l)$, and $\alpha_{2}, \ldots, \alpha_{k+l} \in \Omega$. We put:

1. For any $2 \leqslant i \leqslant k+l$ :

$$
\begin{aligned}
& L_{\sigma}(i)=\left\{\begin{array}{l}
\alpha_{k+1} \text { if } i \leqslant \sigma(1), \\
\alpha_{p} \text { if } \sigma(p-1)<i \leqslant \sigma(p), \text { with } 2 \leqslant p \leqslant k, \\
\varnothing \text { if } i>\sigma(k) ;
\end{array}\right. \\
& R_{\sigma}(i)=\left\{\begin{array}{l}
\alpha_{k+1} \text { if } i \leqslant \sigma(k+1), \\
\alpha_{p} \text { if } \sigma(p-1)<i \leqslant \sigma(p), \text { with } k+2 \leqslant p \leqslant k+l, \\
\varnothing \text { if } i>\sigma(k+l) .
\end{array}\right.
\end{aligned}
$$

2. For any $2 \leqslant i \leqslant k+l$,

$$
D_{\sigma}(i)=\left\{\begin{array}{l}
L_{\sigma}(i) \leftarrow R_{\sigma}(i) \text { if } \sigma^{-1}(i) \leqslant k, \\
L_{\sigma}(i) \rightarrow R_{\sigma}(i) \text { if } \sigma^{-1}(i)>k,
\end{array}\right.
$$

with the convention $\alpha \leftarrow \varnothing=\varnothing \rightarrow \alpha=\alpha$ for any $\alpha \in \Omega$.

Then:

$$
D_{\sigma}^{k, l}\left(\alpha_{2}, \ldots, \alpha_{k+l}\right)=\left(D_{\sigma}(2), \ldots, D_{\sigma}(k+l)\right) .
$$

Proof. Induction on $k+l$.

Remark 6 . Working with $\Omega$ reduced to a single element, we obtain the dual description of the coproduct of the Hopf algebra $\mathcal{Y}$ Sym described in [1].

Corollary 35. Let $\Omega$ be a set. We work in $\mathbf{E D S}(\Omega)$. For any $k, l \geqslant 0$, for any $\sigma \in \operatorname{sh}(k, l)$ :

$$
D_{\sigma}^{k, l}\left(\alpha_{2}, \ldots, \alpha_{k+l}\right)=\left(\alpha_{\sigma^{-1}(2)}, \ldots, \alpha_{\sigma^{-1}(k+l)}\right),
$$

with the convention $\alpha_{1}=\alpha_{k+1}$.

Proof. Let $2 \leqslant i \leqslant k+l$. If $\sigma^{-1}(i) \leqslant k$, then $D_{\sigma}(i)=L_{\sigma}(i)=\alpha_{\sigma^{-1}(i)}$. If $\sigma^{-1}(i)>k$, then $D_{\sigma}(i)=R_{\sigma}(i)=\alpha_{\sigma^{-1}(i)}$. 


\subsection{On typed words}

Remark that:

$$
\mathbb{K} \Omega \otimes \operatorname{Sh}_{\Omega}^{+}(V)=\bigoplus_{n=1}^{\infty}(\mathbb{K} \Omega)^{\otimes n} \otimes V^{\otimes n} .
$$

Proposition 36. Let $\Omega$ be an EDS, and $V$ be a vector space. For any $\alpha_{2}, \ldots, \alpha_{k+l} \in \Omega$, for any $v_{1}, \ldots, v_{k+l} \in V$ :

$$
\begin{aligned}
& \alpha_{2} \ldots \alpha_{k} \otimes v_{1} \ldots v_{k}<_{\alpha_{k+1}} \alpha_{k+2} \ldots \alpha_{k+l} \otimes v_{k+1} \ldots v_{k+l} \\
& =\sum_{\sigma \in \mathbf{s} \mathbf{h}_{<}(k, l)} D_{\sigma}^{k, l}\left(\alpha_{2}, \ldots, \alpha_{k+l}\right) \otimes v_{\sigma^{-1}(1)} \ldots v_{\sigma^{-1}(k+l)}, \\
& \alpha_{2} \ldots \alpha_{k} \otimes v_{1} \ldots v_{k}>_{\alpha_{k+1}} \alpha_{k+2} \ldots \alpha_{k+l} \otimes v_{k+1} \ldots v_{k+l} \\
& =\sum_{\sigma \in \mathbf{s h}(k, l)} D_{\sigma}^{k, l}\left(\alpha_{2}, \ldots, \alpha_{k+l}\right) \otimes v_{\sigma^{-1}(1)} \ldots v_{\sigma^{-1}(k+l)} .
\end{aligned}
$$

Proof. Similar as the proof of Proposition 33

\section{$5 \quad$ Hopf algebraic structure}

\subsection{Existence of dendriform bialgebraic structures}

Let us recall the notion of dendriform bialgebra introduced by Loday and Ronco [9, 10, 11, 12]:

Definition 37. A dendriform bialgebra is a family $(A,<,>, \Delta)$, where $(A,<,>)$ is a dendriform algebra, $(A, \Delta)$ a coassociative coalgebra (not necessarily counitary) such that, for any $x, y \in A$ :

$$
\begin{aligned}
& \Delta(x<y)=x \otimes y+x^{\prime}<y \otimes x^{\prime \prime}+x^{\prime} \otimes x^{\prime \prime} \cdot y+x<y^{\prime} \otimes y^{\prime \prime}+x^{\prime}<y^{\prime} \otimes x^{\prime \prime} \cdot y^{\prime \prime}, \\
& \Delta(x>y)=y \otimes x+x^{\prime}>y \otimes x^{\prime \prime}+y^{\prime} \otimes x \cdot y^{\prime \prime}+x>y^{\prime} \otimes y^{\prime \prime}+x^{\prime}>y^{\prime} \otimes x^{\prime \prime} \cdot y^{\prime \prime},
\end{aligned}
$$

where $=<+>$ is the associative product associated to $(A,<,>)$. We use Sweedler's notations $\Delta(a)=a^{\prime} \otimes a^{\prime \prime}$ for any $a \in A$.

Proposition 38. Let $\Omega$ be an extended disasociative semigroup. If there exists a nonzero graded $\Omega$-dendriform algebra $A$, with $A_{0}=(0)$, with a homogeneous coproduct $\Delta$ making $\mathbb{K} \Omega \otimes A a$ dendriform bialgebra, then $\varphi_{\leftarrow}$ and $\varphi_{\rightarrow}$ are injective.

Proof. Let $a$ be a nonzero element of $A$ of minimal degree $n$. As $n \geqslant 0$, necessarily, for any $\alpha \in \Omega$,

$$
\Delta(\alpha \otimes a)=0 .
$$

Let $\alpha, \beta, \alpha^{\prime}, \beta^{\prime} \in \Omega$, such that $\varphi_{\leftarrow}(\alpha, \beta)=\varphi_{\leftarrow}\left(\alpha^{\prime}, \beta^{\prime}\right)$. Then, in $\mathbb{K} \Omega \otimes A$ :

$$
\alpha \otimes a<\beta \otimes a=\alpha \leftarrow \beta \otimes a<_{\alpha \triangleleft \beta} a=\alpha^{\prime} \leftarrow \beta^{\prime} \otimes a<_{\alpha^{\prime} \triangleleft \beta^{\prime}} a=\alpha^{\prime} \otimes a<\beta^{\prime} \otimes a .
$$

Hence, by the compatibility between $\Delta$ and $<$ :

$$
\Delta(\alpha \otimes a<\beta \otimes a)=(\alpha \otimes a) \otimes(\beta \otimes a)=\Delta\left(\alpha^{\prime} \otimes a<\beta^{\prime} \otimes a\right)=\left(\alpha^{\prime} \otimes a\right) \otimes\left(\beta^{\prime} \otimes a\right) .
$$

As $a \neq 0,(\alpha, \beta)=\left(\alpha^{\prime}, \beta^{\prime}\right)$. Using the compatibility between $\Delta$ and $>$, we obtain that $\varphi \rightarrow$ is injective.

Proposition 39. If $\Omega$ is a nondegenerate EDS, there exists a unique coproduct $\Delta$ on $\mathbb{K} \Omega \otimes \mathbb{K} \mathcal{T}_{\Omega}^{+}$, making it a dendriform bialgebra, such that:

$$
\forall \alpha \in \Omega, \quad \Delta(\alpha \otimes Y)=0 .
$$

Moreover, this dendriform bialgebra is graded by the number of internal vertices. 
Proof. If $\Omega$ is nondegenerate, by Proposition $19, \mathbb{K} \Omega \otimes \mathbb{K} \mathcal{T}_{\Omega}^{+}$is freely generated, as a dendriform algebra, by the elements $\alpha \otimes Y$. From [11, 9, 10, 12, 1], such a $\Delta$ exists and is unique.

Proposition 40. Let $\Omega$ be a nondegenerate EDS and let $V$ be a nonzero vector space. The following conditions are equivalent:

1. There exists a unique coproduct on $\mathbb{K} \Omega \otimes \operatorname{Sh}_{\Omega}^{+}(V)$, making it a dendrifrom bialgebra, such that for any $v \in V$, for any $\alpha \in \Omega, \Delta(\alpha \otimes v)=0$.

2. $\Omega$ is commutative.

Proof. 1. $\Longrightarrow 2$. Let $(\alpha, \beta) \in \Omega^{2}$. As $\Omega$ is nondegenerate, there exists a unique $\left(\alpha^{\prime}, \beta^{\prime}\right) \in \Omega^{2}$, such that $\varphi_{\leftarrow}(\alpha, \beta)=\varphi_{\rightarrow}\left(\alpha^{\prime}, \beta^{\prime}\right)$. Let $v$ be a nonzero element of $V$. By construction of $\left(\alpha^{\prime}, \beta^{\prime}\right)$ :

$\alpha \otimes v<\beta \otimes v=\alpha \leftarrow \beta \otimes(\alpha \triangleleft \beta \otimes v v)=\alpha^{\prime} \rightarrow \beta^{\prime} \otimes\left(\alpha^{\prime} \triangleright \beta^{\prime} \otimes v v\right)=\alpha^{\prime} \otimes v>\beta^{\prime} \otimes v$.

Hence:

$\Delta(\alpha \otimes v<\beta \otimes v)=(\alpha \otimes v) \otimes(\beta \otimes v)=\Delta\left(\alpha^{\prime} \otimes v>\beta^{\prime} \otimes v\right)=\left(\beta^{\prime} \otimes v\right) \otimes\left(\alpha^{\prime} \otimes v\right)$.

As $v \neq 0,\left(\alpha^{\prime}, \beta^{\prime}\right)=(\beta, \alpha)$, so, by definition of $\left(\alpha^{\prime}, \beta^{\prime}\right)$ :

$$
\beta \rightarrow \alpha=\alpha \leftarrow \beta, \quad \beta \triangleright \alpha=\alpha \triangleleft \beta .
$$

Therefore, $\Omega$ is commutative.

2. $\Longrightarrow 1$. By Proposition 20, $\mathbb{K} \Omega \otimes \operatorname{Sh}_{\Omega}^{+}(V)$ is freely generated, as a commutative dendriform algebra, by $\mathbb{K} \Omega \otimes V$. From [1, 11, 4], such a $\Delta$ exists and is unique.

\subsection{Combinatorial description of the coproducts on typed trees}

Let us generalize the combinatorial description of the coproduct given in [8]. We work with $\mathbb{K} \Omega \otimes \mathcal{T}_{\Omega}^{+}$where $\Omega$ is a nondegenerate EDS. We shall use the notations of Proposition 6 .

Notations 4. 1. For any $\alpha \in \Omega$, let $\varphi_{\alpha}^{\mathbf{v}}$ and $\varphi_{\alpha}^{\vee}: \Omega \longrightarrow \Omega$ defined by:

$$
\varphi_{\alpha}^{\triangleleft}(\beta)=\beta \hookrightarrow \alpha, \quad \varphi_{\alpha}^{\triangleright}(\beta)=\alpha \bullet \beta .
$$

2. Let $T \in \mathcal{T}_{\Omega}^{+}$and let $\alpha_{0} \in \Omega$. Let us choose an internal edge $e$ of $T$.

(a) We denote by $T_{e}$ the typed plane binary subtree of $T$ formed by all the vertices of $T$ which are born from $e$.

(b) Let $e_{1}, \ldots, e_{k}$ be the internal edges on the unique path in $T$ from its root to the extremity of $e$; in particular, $e_{k}=e$. For any $i$, let $\alpha_{i}$ be the type of $e_{i}$, and:

- $\boldsymbol{\square}_{i}=\triangleleft$ if $e_{i}$ is a right edge;

- $\mathbf{\square}_{i}=\boldsymbol{\rightarrow}$ if $e_{i}$ is a left edge.

We then put:

$$
T_{e}\left(\alpha_{0}\right)=\varphi_{\alpha_{k}}^{\mathbf{m}_{k}} \circ \ldots \circ \varphi_{\alpha_{1}}^{\mathbf{m}_{1}}\left(\alpha_{0}\right) \otimes T_{e} \in \mathbb{K} \Omega \otimes \mathbb{K} \mathcal{T}_{\Omega}^{+} .
$$

Definition 41. Let $T \in \mathcal{T}_{\Omega}^{+}$.

1. A cut of $T$ is a nonempty subset $c$ of the set of internal edges of $T$.

2. A cut of $T$ is admissible if any path in the tree meets at most one element of $c$. The set of admissible cuts of $T$ is denoted by $\operatorname{Adm}(T)$. Note that if $c$ is an admissible cut of $T$, its elements are naturally ordered from left to right, and we shall write $c=\left\{e_{1}<\ldots<e_{k}\right\}$. 
3. Let $c$ be an admissible cut of $T$. The typed plane binary subtree obtained from $T$ by deleting $T_{e}$ for any $e \in c$ is denoted by $R^{c}(T)$.

Proposition 42. Let $\Omega$ be a nondegenerate $E D S$, and let $T \in \mathcal{T}_{\Omega}^{+}$and $\alpha \in \Omega$. Then:

$$
\Delta(\alpha \otimes T)=\sum_{c=\left\{e_{1}<\ldots<e_{k}\right\} \in \operatorname{Adm}(T)}\left(\alpha \otimes R^{c}(T)\right) \otimes\left(T_{e_{1}}(\alpha) \cdot \ldots \cdot T_{e_{k}}(\alpha)\right),
$$

where $\cdot=<+>$.

Proof. First step. Let us first prove that, for any admissible cut $c \in \operatorname{Adm}(T)$, there exists a tree $\iota_{c}\left(\alpha \otimes R^{c}(T)\right)$ obtained from $\alpha \otimes R^{c}(T)$ by an action on the types of the internal edges and on $\alpha$, such that:

$$
\Delta(\alpha \otimes T)=\sum_{c=\left\{e_{1}<\ldots<e_{k}\right\} \in \operatorname{Adm}(T)} \iota_{c}\left(\alpha \otimes R^{c}(T)\right) \otimes\left(T_{e_{1}}(\alpha) \cdot \ldots \cdot T_{e_{k}}(\alpha)\right) .
$$

For any trees $T_{1}, T_{2}$, for any $\alpha, \beta \in \Omega$ :

$$
\begin{aligned}
& \alpha \otimes \mid \bigvee_{\varnothing, \gamma} T_{2}=\beta \curvearrowleft \gamma \otimes Y<\beta \triangleleft \gamma \otimes T_{2}, \\
& \alpha \otimes T_{1} \bigvee_{\beta, \varnothing} \mathrm{I}=\beta \triangleright \alpha \otimes T_{1}>\beta \smile \alpha \otimes Y, \\
& \alpha \otimes T_{1} \bigvee_{\beta, \gamma} T_{2}=\beta \triangleright \alpha \otimes T_{1}>(\beta \smile \alpha) \curvearrowleft \gamma \otimes Y \prec \alpha \triangleleft \gamma \otimes T_{2} .
\end{aligned}
$$

Remark that if $T=T_{1} \bigvee_{\alpha, \beta} T_{2}$, then any admissible cut $c$ of $T$ is of the form $c_{1} \sqcup c_{2}$, where $c_{i}$ is either an admissible cut, or the empty cut, or the total cut (which means that $R^{c}(T)=\varnothing$ ), of $c_{i}$; at least $c_{1}$ or $c_{2}$ is not empty. Then $\iota_{c}\left(\alpha \otimes R^{c}(T)\right)$ is inductively defined by:

- If $c$ is total, then $\iota_{c}\left(\alpha \otimes R^{c}(T)\right)=\varnothing \otimes$ ।.

- If $c$ is empty, then $\iota_{c}\left(\alpha \otimes R^{c}(T)\right)=\alpha \otimes T$.

- If $c=c_{1} \sqcup c_{2}$, then:

$$
\iota_{c}\left(\alpha \otimes R^{c}(T)\right)=\iota_{c_{1}}\left(\beta \triangleright \alpha \otimes T_{1}\right)>(\beta \frown \alpha) \curvearrowleft \gamma \otimes Y \prec \iota_{c_{2}}\left(\alpha \triangleleft \gamma \otimes T_{2}\right) .
$$

Using the compatibilities between the dendriform products and the coproducts, we obtain the result by induction on the number of internal vertices of $T$.

Second step. Let us prove that $\iota_{c}\left(\alpha \otimes R^{c}(T)\right)=\alpha \otimes R^{c}(T)$ by induction on the number $n$ of internal vertices of $T$. It is obvious if $n=1$. Otherwise, we put $T=T_{1} \bigvee_{\beta, \gamma} T_{2}$, and $c=c_{1} \sqcup c_{2}$. Then, using the induction hypothesis on $T_{1}$ and $T_{2}$ :

$$
\begin{aligned}
\iota_{c}\left(\alpha \otimes R^{c}(T)\right) & =\iota_{c_{1}}\left(\beta \triangleright \alpha \otimes R^{c_{1}}\left(T_{1}\right)\right)>(\beta \frown \alpha) \curvearrowleft \gamma \otimes Y<\iota_{c_{2}}\left(\alpha \triangleleft \gamma \otimes R^{c_{2}}\left(T_{2}\right)\right) \\
& =\beta \bullet \alpha \otimes R^{c_{1}}\left(T_{1}\right)>(\beta \frown \alpha) \curvearrowleft \gamma \otimes Y \prec \alpha \triangleleft \gamma \otimes R^{c_{2}}\left(T_{2}\right) \\
& =(\beta \bullet \alpha) \rightarrow((\beta \frown \alpha) \curvearrowleft \gamma) \otimes R^{c_{1}}\left(T_{1}\right)>(\beta \bullet \alpha) \triangleright((\beta \neg \alpha) \curvearrowleft \gamma) Y<\alpha \triangleleft \gamma \otimes R^{c_{2}}\left(T_{2}\right) .
\end{aligned}
$$

By (34):

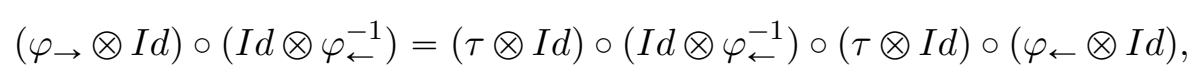

so, for any $\alpha^{\prime}, \beta^{\prime}, \gamma^{\prime} \in \Omega$ :

$$
\left(\alpha^{\prime} \rightarrow\left(\beta^{\prime} \curvearrowleft \gamma^{\prime}\right), \alpha^{\prime} \triangleright\left(\beta^{\prime} \curvearrowleft \gamma^{\prime}\right), \beta^{\prime} \triangleleft \gamma^{\prime}\right)=\left(\left(\alpha^{\prime} \rightarrow \beta^{\prime}\right) \curvearrowleft \gamma^{\prime}, \alpha^{\prime} \triangleright \beta^{\prime},\left(\alpha^{\prime} \rightarrow \beta^{\prime}\right) \triangleleft \gamma^{\prime}\right) .
$$


For $\alpha^{\prime}=\beta \triangleright \alpha, \beta^{\prime}=\beta \frown \alpha$ and $\gamma^{\prime}=\gamma$, we obtain:

$$
\begin{aligned}
& (\beta \triangleright \alpha) \rightarrow((\beta \smile \alpha) \curvearrowleft \gamma)=((\beta \triangleright \alpha) \rightarrow(\beta \smile \alpha)) \curvearrowleft \gamma=\alpha \curvearrowleft \gamma \\
& (\beta \triangleright \alpha) \triangleright((\beta \smile \alpha) \curvearrowleft \gamma)=(\beta \triangleright \alpha) \triangleright(\beta \smile \alpha)=\beta .
\end{aligned}
$$

Hence:

$$
\begin{aligned}
\iota_{c}\left(\alpha \otimes R^{c}(T)\right) & =\alpha \curvearrowleft \gamma \otimes R^{c_{1}}\left(T_{1}\right)>_{\beta} Y<\alpha \triangleleft \gamma \otimes R^{c_{2}}\left(T_{2}\right) \\
& =\alpha \curvearrowleft \gamma \otimes R^{c_{1}}\left(T_{1}\right) \bigvee_{\beta, \varnothing} \mid<\alpha \triangleleft \gamma \otimes R^{c_{2}}\left(T_{2}\right) \\
& =(\alpha \curvearrowleft \gamma) \leftarrow(\alpha \triangleleft \gamma) \otimes R^{c_{1}}\left(T_{1}\right) \bigvee_{\beta, \varnothing} \mid<(\alpha \curvearrowleft \gamma) \triangleleft(\alpha \triangleleft \gamma) R^{c_{2}}\left(T_{2}\right) \\
& =\alpha \otimes R^{c_{1}}\left(T_{1}\right) \bigvee_{\beta, \varnothing} \mid \prec_{\gamma} R^{c_{2}}\left(T_{2}\right) \\
& =\alpha \otimes R^{c_{1}}\left(T_{1}\right) \bigvee_{\beta, \gamma} R^{c_{2}}\left(T_{2}\right) \\
& =\alpha \otimes R^{c}(T) .
\end{aligned}
$$

Hence, $\iota_{c}\left(\alpha \otimes R^{c}(T)\right)=\alpha \otimes R^{c}(T)$ for any admissible cut of any tree $T$.

Remark 7. Working with $\Omega$ reduced to a single element, we obtain the dual description of the product of the Hopf algebra $\mathcal{Y}$ Sym described in [1].

Example 14. 1. Let $(\Omega, \leftarrow, \rightarrow)$ be a diassociative monoid. We assume that $\operatorname{EDS}(\Omega, \leftarrow, \rightarrow)$ is nondegenerate. In this case, for any $\alpha, \beta \in \Omega, \varphi_{\alpha}^{\triangleleft}(\beta)=\varphi_{\alpha}^{\triangleright}(\beta)=\alpha$. Hence, for any edge $e$ of a given tree $T$, of type $\alpha_{e}$, for any $\alpha_{0} \in \Omega$ :

$$
T_{e}\left(\alpha_{0}\right)=\alpha_{e} \otimes T_{e}
$$

2. Let $(\Omega, \star)$ be a group. In $\operatorname{EDS}^{*}(\Omega, \star)$, for any $\alpha, \beta \in \Omega, \varphi_{\alpha}^{\triangleleft}(\beta)=\varphi_{\alpha}^{\triangleright}(\beta)=\beta \star \alpha$. Hence, for any edge $e$ of a given tree $T$, of type $\alpha_{e}$, for any $\alpha_{0} \in \Omega$ :

$$
T_{e}\left(\alpha_{0}\right)=\alpha_{0} \star \ldots \star \alpha_{k} \otimes T_{e} .
$$

\subsection{Combinatorial description of the coproducts on typed words}

Proposition 43. Let $\Omega$ be a nondegenerate commutative EDS. We use the notations of Proposition 6. In the dendriform bialgebra $\mathbb{K} \Omega \otimes \operatorname{Sh}_{\Omega}^{+}(V)$, for any $\alpha_{1}, \ldots, \alpha_{n} \in \Omega$, for any $v_{1}, \ldots, v_{n} \in V$ :

$$
\begin{aligned}
& \Delta\left(\alpha_{1} \ldots \alpha_{n} \otimes v_{1} \ldots v_{n}\right) \\
& =\sum_{i=1}^{n-1}\left(\alpha_{1} \ldots \alpha_{i} \otimes v_{1} \ldots v_{i}\right) \otimes\left(\left(\alpha_{1} \triangleleft \ldots \triangleleft \alpha_{i+1}\right) \alpha_{i+2} \ldots \alpha_{n} \otimes v_{i+1} \ldots v_{n}\right) .
\end{aligned}
$$

Proof. We work with the $\Omega$-dendriform algebra $A$ of $\Omega$-typed plane binary trees which internal vertices are decorated by $V$. All the results presented for nondecorated trees can be extended to this context. For any $v \in V$, we denote by $Y(v)$ the plane binary tree $Y$ which unique internal vertex is decorated by $v$. By freeness, there exists a unique $\Omega$-dendriform algebra morphism $\Phi$ from $A$ to $\operatorname{Sh}_{\Omega}^{+}(V)$, sending $Y(v)$ to $v$ for any $v \in V$. It naturally induces a dendriform algebra morphism from $\mathbb{K} \Omega \otimes A$ to $\mathbb{K} \Omega \otimes \operatorname{Sh}_{\Omega}^{+}(V)$, also denoted by $\Phi$. For any $v \in V$, any $\alpha \in \Omega, \alpha \otimes Y(v)$ is primitive in $\mathbb{K} \Omega \otimes A$, and $\alpha \otimes v$ is primitive in $\mathbb{K} \Omega \otimes \operatorname{Sh}_{\Omega}^{+}(V)$ : this implies that $\Phi$ is a dendriform bialgebra morphism. 
Let us introduce some notations. For any $n \geqslant n$ and $\alpha_{2}, \ldots, \alpha_{n} \in \Omega, R_{n}\left(\alpha 2 \ldots \alpha_{n}\right)$ is inductively defined by:

$$
R_{1}=Y, \quad R_{n}\left(\alpha_{2} \ldots \alpha_{n}\right)=1 \bigvee_{\varnothing, \alpha_{2}} R_{n-1}\left(\alpha_{3} \ldots \alpha_{n}\right) \text { if } n \geqslant 2
$$

Note that the underlying plane binary tree of $R_{n}\left(\alpha_{2} \ldots \alpha_{n}\right)$ is a right comb. For example:

$$
R_{2}\left(\alpha_{2}\right)=Y^{1}\left(\alpha_{2}\right), \quad R_{3}\left(\alpha_{2} \alpha_{3}\right)=Y_{1}^{1^{2}}\left(\alpha_{2}, \alpha_{3}\right) .
$$

For $v_{1}, \ldots, v_{n} \in V$ we denote by $R_{n}\left(\alpha_{2} \ldots \alpha_{n} ; v_{1} \ldots v_{n}\right)$ the $\Omega$-typed plane binary tree by giving the $n$ internal vertices of $R_{n}\left(\alpha_{2} \ldots \alpha_{n}\right)$, naturally ordered starting from the root, the decorations $v_{1}, \ldots, v_{n}$.

By definition of the products on trees:

$$
\begin{aligned}
& (\alpha \curvearrowleft \beta) \otimes Y(v) \prec(\alpha \triangleleft \beta) \otimes R_{n}\left(\alpha_{2} \ldots \alpha_{n} ; v_{1} \ldots v_{n}\right) \\
& =(\alpha \curvearrowleft \beta) \leftarrow(\alpha \triangleleft \beta) \otimes Y(v) \prec_{(\alpha \curvearrowleft \beta) \triangleleft(\alpha \triangleleft \beta)} R_{n}\left(\alpha_{2} \ldots \alpha_{n} ; v_{1} \ldots v_{n}\right) \\
& =\alpha \otimes Y(v) \prec_{\beta} R_{n}\left(\alpha_{2} \ldots \alpha_{n} ; v_{1} \ldots v_{n}\right) \\
& =\alpha \otimes । \bigvee_{\varnothing, \beta} R_{n}\left(\alpha_{2} \ldots \alpha_{n} ; v_{1} \ldots v_{n}\right) \\
& \quad \alpha \otimes R_{n+1}\left(\beta \alpha_{2} \ldots \alpha_{n} ; v v_{1} \ldots v_{n}\right) .
\end{aligned}
$$

An easy induction proves that:

$$
\Phi\left(\alpha_{1} \otimes R_{n}\left(\alpha_{2} \ldots \alpha_{n} ; v_{1} \ldots v_{n}\right)\right)=\alpha_{1} \ldots \alpha_{n} \otimes v_{1} \ldots v_{n} .
$$

The admissible cuts of $R_{n}$ are the cuts of a single internal edge: hence, by Proposition 42 ,

$$
\begin{aligned}
& \Delta\left(\alpha_{1} \otimes R_{n}\left(\alpha_{2} \ldots \alpha_{n} ; v_{1} \ldots v_{n}\right)\right) \\
& =\sum_{i=1}^{n-1}\left(\alpha_{1} \otimes R_{i}\left(\alpha_{2} \ldots \alpha_{i} ; v_{1} \ldots v_{i}\right)\right) \otimes\left(\varphi_{\alpha_{i+1}}^{\mathbf{A}} \circ \ldots \circ \varphi_{\alpha_{2}}^{\triangleleft}\left(\alpha_{1}\right) \otimes R_{n-i}\left(\alpha_{i+2} \ldots \alpha_{n} ; v_{i+1} \ldots v_{n}\right)\right) \\
& =\sum_{i=1}^{n-1}\left(\alpha_{1} \otimes R_{i}\left(\alpha_{2} \ldots \alpha_{i} ; v_{1} \ldots v_{i}\right)\right) \otimes\left(\alpha_{1} \bullet \ldots \triangleleft \alpha_{i+1} \otimes R_{n-i}\left(\alpha_{i+2} \ldots \alpha_{n} ; v_{i+1} \ldots v_{n}\right)\right) .
\end{aligned}
$$

The result is obtained by application of $\Phi$.

\section{References}

[1] Marcelo Aguiar and Frank Sottile, Structure of the Loday-Ronco Hopf algebra of trees, J. Algebra 295 (2006), no. 2, 473-511. MR 2194965

[2] Murray R. Bremner and Vladimir Dotsenko, Algebraic operads, CRC Press, Boca Raton, FL, 2016, An algorithmic companion. MR 3642294

[3] Y. Bruned, M. Hairer, and L. Zambotti, Algebraic renormalisation of regularity structures, Invent. Math. 215 (2019), no. 3, 1039-1156. MR 3935036

[4] L. Foissy, Les algèbres de Hopf des arbres enracinés décorés. II, Bull. Sci. Math. 126 (2002), no. 4, 249-288. MR 1909461

[5] Loïc Foissy, Algebraic structures on typed decorated rooted trees, arXiv:1811.07572, 2018. 
[6] Loïc Foissy and Frédéric Patras, Lie theory for quasi-shuffle bialgebras, arXiv:1605.02444, 2016.

[7] Xing Gao, Li Guo, and Yuanyuan Zhang, Matching Rota-Baxter algebras, matching dendriform algebras and matching pre-Lie algebras, arxiv:1909.10577, 2019.

[8] Xing Gao and Yuanyuan Zhang, Hopf algebras of planar binary trees: an operated algebra approach, arxiv:1905.09430, 2019.

[9] Jean-Louis Loday and María Ronco, Trialgebras and families of polytopes, Homotopy theory: relations with algebraic geometry, group cohomology, and algebraic $K$-theory, Contemp. Math., vol. 346, Amer. Math. Soc., Providence, RI, 2004, pp. 369-398. MR 2066507

[10] Combinatorial Hopf algebras, Quanta of maths, Clay Math. Proc., vol. 11, Amer. Math. Soc., Providence, RI, 2010, pp. 347-383. MR 2732058

[11] Jean-Louis Loday and María O. Ronco, Hopf algebra of the planar binary trees, Adv. Math. 139 (1998), no. 2, 293-309. MR 1654173

[12] María Ronco, A Milnor-Moore theorem for dendriform Hopf algebras, C. R. Acad. Sci. Paris Sér. I Math. 332 (2001), no. 2, 109-114. MR 1813766

[13] M. P. Schützenberger, Sur une propriété combinatoire des algèbres de Lie libres pouvant être utilisée dans un problème de mathématiques appliquées, Séminaire Dubreil-Jacotin Pisot (Algèbre et théorie des nombres) (1958/59).

[14] Yuanyuan Zhang, Xing Gao, and Dominique Manchon, Free (tri)dendriform family algebras, arXiv:1909.08946, 2019. 